\title{
An assessment of the use of drug and non-drug interventions in the treatment of Ichthyophthirius multifiliis Fouquet, 1876, a protozoan parasite of freshwater fish
}

\author{
S. M. PICÓN-CAMACHO ${ }^{1}$, M. MARCOS-LOPEZ ${ }^{2}$, J. E. BRON ${ }^{1}$ and A. P. SHINN ${ }^{1}$ \\ ${ }^{1}$ Institute of Aquaculture, University of Stirling, FK9 4LA Stirling, UK \\ ${ }^{2}$ Marine Laboratory, 375 Victoria Rd, AB11 9DB Aberdeen, UK
}

(Received 17 fune 2011; revised 12 September 2011; accepted 16 September 2011; first published online 14 November 2011)

\begin{abstract}
SUMMARY
Infection by the ciliate protozoan Ichthyophthirius multifiliis Fouquet, 1876 causes significant economic losses in freshwater aquaculture worldwide. Following the ban on the use of malachite green for treating food fish, there has been extensive research aimed at identifying suitable replacements. In this paper we critically assess drug and non-drug interventions, which have been tested for use or have been employed against this parasite and evaluate possibilities for their application in farm systems. Current treatments include the administration of formaldehyde, sodium chloride (salt), copper sulphate and potassium permanganate. However, purportedly more environmentally friendly drugs such as humic acid, potassium ferrate (VI), bronopol and the peracetic acid-based products have recently been tested and represent promising alternatives. Further investigation, is required to optimize the treatments and to establish precise protocols in order to minimize the quantity of drug employed whilst ensuring the most efficacious performance. At the same time, there needs to be a greater emphasis placed on the non-drug aspects of management strategies, including the use of non-chemical interventions focusing on the removal of free-swimming stages and tomocysts of I. multifiliis from farm culture systems. Use of such strategies provides the hope of more environmentally friendly alternatives for the control of I. multifiliis infections.
\end{abstract}

Key words: Ichthyophthirius multifiliis, whitespot, drug, treatment, ciliate, parasite.

\section{INTRODUCTION}

The freshwater protozoan parasite of fish, Ichthyophthirius multifiliis Fouquet 1876, also known as 'fish whitespot', continues to impact wild and cultured fish populations worldwide and places an economic burden on global freshwater finfish aquaculture.

The ciliate protozoan I. multifiliis is one of the most important freshwater pathogens affecting the aquaculture and ornamental fish industries. In part, its impact stems from its low host specificity, allowing it to infect a wide range of fish species, including commercially important species such as channel catfish (Ictalurus punctatus Rafinesque 1818) and rainbow trout (Oncorhynchus mykiss Walbaum 1792) (see Valtonen and Koskivaara, 1994; Noble and Summerfelt, 1996; Buchmann and Bresciani, 1997; Rintamäki-Kinnunen and Valtonen, 1997; Matthews, 2005; Jørgensen et al. 2009). It has a direct life cycle, which is temperature dependent such that the warmer the water temperature the faster the life

* Corresponding author: Gulf Coast Research Laboratory, University of Southern Mississippi, Ocean Springs, MS 39564, USA. Tel: +228 8188807 . E-mail: sarapicon@ yahoo.es cycle completes. The life cycle involves 4 different stages: (1) the trophont, which resides within the surface epithelium of gills, fins and other body surfaces; (2) the protomont, a free-swimming stage that exits the fish and settles on the substrate to become the encysted tomocyst stage (3) which in turn repeatedly divides by binary fission to produce tomites which are released to the water column. Tomites differentiate into the infective stage (4) the theront, which needs to find a host within a short window to successfully complete the life cycle by penetrating the epidermis and developing into the trophont stage before it dies (Lom and Dyková, 1992; Matthews, 2005). Theronts can survive for up to $92 \mathrm{~h}$ at low water temperatures; their survival being inversely proportional to the ambient water temperature (Wagner, 1960; Aihua and Buchmann, 2001).

On farms, the most common approaches to treat this ciliate is through the use of either short (e.g. $30 \mathrm{~min}-4 \mathrm{~h}$ in tanks, raceways and flow-through systems) or long (e.g. 7-15 days in pond culture) duration in-bath treatments which target the freeswimming stages of the parasite (i.e. protomonts and theronts). Of the other two stages, the trophont is protected lying underneath the host surface 
epithelium (Post and Vesely, 1983) whilst the tomocyst is protected by a resistant coat (Ewing et al. 1983) and as such, are rarely susceptible to treatment.

Historically, malachite green (MG) was commonly used for the control of I. multifiliis and a range of other fish diseases (Srivastava et al. 2004) due to its demonstrable efficacy, low cost, ready availability, high stability during storage and high solubility in water (Schnick, 1988; Henderson et al. 1997). This organic (triphenylmethane) dye was favoured for the control of I. multifiliis infections because of its high efficacy against both the free-swimming stages (protomonts and theronts) of the parasite and the feeding parasite stage (trophont) within the fish's epithelium (Wahli et al. 1993; Tieman and Goodwin, 2001; Buchmann et al. 2003). MG and its derivatives (mainly leucomalachite) also display well-documented ecotoxicological effects including cytotoxicity, carcinogenicity, mutagenicity, induction of chromosomal fractures, teratogenicity and respiratory toxicity (Culp and Beland, 1996; Srivastava et al. 2004). Malachite green and its derivatives are also known to be highly persistent in the environment, bio-accumulating in the ecosystem and fish tissues (Henderson et al. 1997). Although the use of MG has never been licensed by the US Food and Drug Administration (FDA), its use in food products was initially permitted under an 'investigational new animal drug' status (Alderman, 1985). This status was revoked in 1983 and MG was listed as a priority chemical for toxicity and carcinogenicity testing (Culp and Beland, 1996; Culp, 2004). Similarly, in Canada the use of MG and the presence of its derivatives in food animals are not permitted and its continued use was advised against in 1992 when MG was classified as a class II health hazard (Canadian Food Inspection Agency 2010). Its use within the European Union has been subsequently banned in 2000 under EC directive 90/676/EEC; article 14, regulation 2377/90/EEC.

As a consequence of the widespread ban, enforced restrictions imposed on the use of $\mathrm{MG}$ and the concerns regarding the presence of derivatives in food-products (Herber, 2009), there has been extensive research in the last few decades focusing on the provision of alternative, effective and environmentally friendly products and management techniques for controlling I. multifiliis infections. Despite the global effort, no clear alternative management strategies have yet emerged. There is a strong commercial and scientific need for providing a critical summary of tested candidate and applied drugs but also an assessment of the potential of other management strategies to prove efficacious against I. multifiliis infections. It has been nearly 30 years since the last major reviews were published examining the use of drugs for the control of I. multifiliis (Cross, 1972; Hoffman and Meyer, 1974; Herwig,
1979) so that this review might be considered to be somewhat overdue.

This paper provides an overview and assessment of the current state of knowledge concerning drugs (compound, dose, duration and efficacy) and physical interventions employed or tested against $I$. multifiliis since the 3 earlier reviews were published. This review seeks to summarize the original research findings and to help identify the most suitable therapy against $I$. multifiliis while highlighting the most promising treatments for further research and application in farm systems.

\section{ASSESSMENT OF CURRENTLY APPLIED}

CHEMOTHERAPIES

A large number of compounds have been tested for efficacy against $I$. multifiliis although relatively few of them have been widely deployed to provide effective control under field conditions. Table 1 provides a detailed list of 116 compounds used to control I. multifiliis under laboratory or field conditions from 1980 onwards. Of the compounds that are listed, all except quinine and some malachite greenbased formulations have been tested against food fish species. These latter treatments, however, that have been evaluated for the ornamental trade, are included to provide a comprehensive overview of all compounds tested for the treatment of $I$. multifiliis. Of those given in Table 1, 18 entries listed by their commercial product name are cross-referenced, and details of their activity given, under their specific compound formulation. Sixteen of the compounds have been assessed by in vitro trials only, while of the remaining 81 compounds tested in vivo, 43 have been tested in-bath challenges and 51 by in-feed presentation. Of those used under field conditions, the most commonly used treatments are: formaldehyde, sodium chloride, copper sulphate, potassium permanganate, chloramine- $\mathrm{T}$, hydrogen peroxide, metronidazole and toltrazuril (Dickerson, 2006; Noga, 2010). Whilst malachite green was previously the most extensively employed treatment, eliminating the protomont, theront and trophont stages, its use has been largely discontinued for food fish, particularly in the EU and the United States.

Some caution, however, should be taken with regard to the treatment efficacies provided in Table 1 , in that these may be the result of how the study was conducted (i.e. natural, multi-age class infections compared to a standard, single age class infection) and/or evaluated (i.e. parasite numbers determined from skin scrapes as opposed to total parasite counts) and therefore the results may have been affected by the differential level of parasitaemia at the time of the treatment on the test and control fish. For efficacious compounds of interest, therefore, details on the treatment conditions used in the original work should be consulted. If the physiological trauma created by 
exiting trophonts is considered as the primary cause of mortality, then a compound that successfully kills trophonts in situ, thereby preventing exit, could be considered efficacious (i.e. a statistically lower number of parasites and host mortality when compared to an appropriate control group).

\section{Formaldehyde}

Formaldehyde has been proven to be very effective at eliminating the free-living stages of the parasite (i.e. protomonts, tomocysts and theronts) (Wahli et al. 1993; Shinn et al. 2005, Lahnsteiner and Weismann, 2007; Heinecke and Buchmann, 2009), however, when used for in vivo baths, fish survival can be compromised (Wahli et al. 1993; Tieman and Goodwin, 2001). Formaldehyde remains one of the most commonly used treatments to control I. multifiliis infections in aquaculture systems (Noga, 2010). However, efficiency is achieved only at high concentrations, which are serially repeatedly applied (i.e. $100 \mathrm{mg} \mathrm{l}^{-1}$ for $30 \mathrm{~min}$ to $1 \mathrm{~h}$ over 10 consecutive days in salmonid farms), such that in flow-through systems with rapid water turn-over, as used for e.g. the intensive production of salmonids, high volumes are required. In addition, the use of formaldehyde has many reported side effects such as reducing the oxygen available in the water by $1 \mathrm{ppm}$ for each 5 ppm of formaldehyde that is used (Cross, 1972; Pillay and Kutty, 2005). This can be particularly problematical in summer when increasing water temperatures both accelerate the life cycle of I. multifiliis and act to cause a concomitant reduction in the oxygen holding capacity of the water. Buchmann et al. (2004) also demonstrated that $O$. mykiss exposed to formaldehyde at concentrations of $200-300 \mathrm{ppm}$ for $1 \mathrm{~h}$ had a reduced mucus production and were thus more susceptible to secondary infections by water moulds and bacteria. Accordingly, when formaldehyde is applied in vivo in the form of baths, fish survival can be compromised (e.g. for O. mykiss exposed to 2 treatments of 25 and $100 \mathrm{mg} \mathrm{l}^{-1}$ of formaldehyde for $1 \mathrm{~h}$ on days 9 and 12 post-infection) (Wahli et al. 1993). Importantly, the effect of water quality parameters on the toxicity of formaldehyde to fish and to I. multifiliis remains poorly characterized (Meinelt et al. 2005). Although formaldehyde is an approved aquacultural therapeutic within the EU (Schlotfeld, 1993, 1998), in 2004 it was re-classified by the WHO International Agency for Research on Cancer as 'carcinogenic to humans' (WHO, 2006). Even though it is quickly metabolized by aquatic organisms and holds a low potential for bio-accumulation (Hohreiter and Rigg, 2001; Duffort et al. 2010), it might be envisaged that formaldehyde could soon be banned due to the hazard it poses to workers handling large volumes of the chemical (Wooster et al. 2005). Given the high volumes of formaldehyde required in a typical farm treatment and the potential toxic risks this chemical poses to both fish stock and the farm workers handling it, the future of formaldehyde as a longterm acceptable and sustainable drug seems unlikely.

\section{Sodium chloride}

Sodium chloride (salt) is the second most commonly used product for the treatment of I. multifiliis infections. The application of a minimum of $2 \cdot 5 \mathrm{~g}^{-1}$ has been proven to reduce protomont and theront survival (Aihua and Buchmann, 2001; Shinn et al. 2005; Lahnsteiner and Weismann, 2007). A treatment regime of $1-5 \mathrm{~g}^{-1}$ salt applied continuously for a minimum period of 7 to 32 days, for example, was able to reduce the number of trophonts establishing on fish (Selosse and Rowland, 1990; Miron et al. 2003; Lahnsteiner and Weismann, 2007; Balta et al. 2008; Mifsud and Rowland, 2008). The use of higher concentrations of salt (e.g. 15-20 $\mathrm{g} \mathrm{l}^{-1}$ ) over short periods of exposure (e.g. 20-60 min), however, was not able to reduce the level of infection (Lahnsteiner and Weismann, 2007; Balta et al. 2008). Additionally, the bath application of salt may be beneficial, in helping the host recover the osmotic imbalance and loss of salts created by exiting trophonts. The incorporation of salt in fish feed has also been explored with contradictory results. Rahkonen and Koski (2002) reported a reduction in infection levels in medicated fish when salt was incorporated at a level of $0 \cdot 3-1 \cdot 0 \%$ and fed for 3 to 11 days. Garcia et al. (2007), however, did not observe any significant reduction in parasite burdens when fish were fed a diet containing $1 \cdot 2-6 \cdot 0 \%$ salt for a period of 30 days. While the use of salt appears to represent an economically viable and safe treatment option for many farm and ornamental fish species, it should be used with caution in certain infected stenohaline freshwater fish species such as channel catfish (Noga, 2010).

\section{Copper sulphate}

Copper sulphate has been shown to be effective at eliminating I. multifiliis in a range of fish species when used at low concentrations (Ling et al. 1993; Schlenk et al. 1998; Goodwin and Straus, 2006; Straus, 2008; Rowland et al. 2009). However, long periods of exposure can lead to toxicity, gill damage and growth suppression (Cardeilhac and Whitaker, 1988; Moore, 2005; Rábago-Castro et al. 2006). Copper has a very low therapeutic index (Boyd, 2005) and its toxicity to both fish host and I. multifiliis is known to vary widely with water chemistry parameters, particularly water alkalinity and hardness (Deilhac and Whitaker, 1988; Straus, 2008; Straus and Meinelt, 2009). Copper sulphate is a recognized algaecide and is known to be toxic to a 
wide range of invertebrate organisms (Boyd, 1990). When added to pond systems, there is a risk of phytoplankton mortality which consequentially might result in lower oxygen levels at night, which in turn compromises the trophic chain on which the fish stock might rely (Noga, 2010). It is vital therefore that its use on a small subsample of the fish stock in the local water is determined before it is applied on a large-scale basis. Particular care should be taken when using this compound in green water pond systems. Future research should be aimed at identifying the range of water quality parameters and concentrations within which this compound is effective against I. multifiliis infections and can be safely administered without risk to fish.

\section{Potassium permanganate}

Potassium permanganate $\left(\mathrm{KMnO}_{4}\right)$ is also commonly used against $I$. multifiliis, mainly in farm pond systems (Brown and Gratzek, 1980; Noga, 2010). Low concentrations (e.g. $0 \cdot 8-1 \cdot 0 \mathrm{mg} \mathrm{l}^{-1}$ ) over short periods of exposure ( $30 \mathrm{~min}$ to $4 \mathrm{~h}$ ) were able to eliminate the theront stage in the water column (Straus and Griffin, 2001). When tested in vivo, low concentrations (e.g. $0 \cdot 25-2 \mathrm{mg} \mathrm{l}^{-1}$ ) require longer periods of exposure (continuously from 6 to 20 days) to significantly decrease the number of trophonts per fish (Tieman and Goodwin, 2001; Straus and Griffin, 2001, 2002). The application of higher concentrations (e.g. 10-20 $\mathrm{mg} \mathrm{l}^{-1}$ ) for $30 \mathrm{~min}$ was found to be toxic to treated fish (Balta et al. 2008). Potassium permanganate is an algaecide which oxidizes organic matter, reducing dissolved oxygen levels; its effects are notable when used in ponds. This compound has a low therapeutic index and can be very toxic when used in waters of a high $\mathrm{pH}$ when it can precipitate on gills leading to high mortalities (Tucker, 1987; Dolezelova et al. 2009; Noga, 2010). Potassium permanganate treatment against $I$. multifiliis shows very low efficacy at concentrations that are not toxic to fish, if the organic loading of the aquatic system is not taken into account. Large quantities of this compound and its continuous application, therefore, are often required to manage infections.

\section{Chloramine- $T$}

Chloramine- $\mathrm{T}$ is an organic chlorine compound, specifically a sodium salt that when mixed with water is a very strong disinfectant (Treves-Brown, 2000; Noga, 2010). When used to treat I. multifiliis stages, chloramine- $T$ has been found to be very effective in vitro for the treatment of both the protomont and theront stages (Shinn et al. 2001). In vivo, however, chloramine- $T$ was effective only when administered at high concentrations (e.g. $100 \mathrm{mg} \mathrm{l}^{-1}$ for $30 \mathrm{~min}$ given over a period of 10 days) (Shinn et al. 2001; Tieman and Goodwin, 2001; Rahkonen and Koski,
2002; Shinn et al. 2003a; Rintamäki-Kinnunen et al. 2005a; Balta et al. 2008). The administration of high doses of chloramine- $\mathrm{T}$ can inflict damage to the gill epithelia and has been reported to affect the development of the swim bladder in young fry (Sanabria et al. 2009). The average lethal time $\left(\mathrm{LT}_{50}\right)$ for a dose of $50 \mathrm{mgl}^{-1}$ chloramine- $\mathrm{T}$ was determined to be 166.8 $\mathrm{min}$ (Powell and Harris, 2004). Although these latter authors suggested that freshwater stages of Atlantic salmon, Salmo salar L., were as sensitive to chloramine-T toxicity as $O$. mykiss, and more sensitive than I. punctatus, the latter showed histopathological changes when exposed daily to $80 \mathrm{mg} \mathrm{l}^{-1}$ in a static immersion bath for $3 \mathrm{~h}$ (Gaikowski et al. 2009). Future work, therefore, should explore the efficacy of using $30 \mathrm{~min}$ baths of chloramine-T ranging between 30 and $80 \mathrm{mgl}^{-1}$ over a period of 10 days (e.g. treatments on days: 1, 4, 7 and 10) (or the full duration of the parasite life cycle as dictated by the ambient water temperature).

\section{Hydrogen peroxide}

Hydrogen peroxide is a powerful oxidizer that has been used under field conditions to control I. multifiliis. Results for its use in in vitro tests against free-living stages of I. multifiliis, however, were disappointing (Shinn et al. 2005; Lahnsteiner and Weismann, 2007), with a $100 \mathrm{mg} \mathrm{l}^{-1}$ treatment for $1 \mathrm{~h}$ effecting only a $15 \%$ mortality of theronts (Shinn et al. unpublished observations). It is not surprising, therefore, that a 20 -day regime of $25 \mathrm{mg} \mathrm{l}^{-1}$ hydrogen peroxide failed to bring about a reduction in the number of trophonts on stock, which consequentially resulted in high mortalities (Tieman and Goodwin, 2001). High doses, however, can cause gill damage leading to fish mortality (especially at high temperatures) (Schmidt et al. 2006; Noga, 2010).

\section{Metronidazole}

Metronidazole has been shown to be very successful at reducing the number of trophonts on infected fish when incorporated into diets (Tojo-Rodriguez and Santamarina-Fernandez, 2001; Tokşen and Nemli, 2010). This compound, which has been shown to be effective in the ornamental fish industry, is currently listed as being 'possibly carcinogenic to humans' by the World Health Organization and has been banned within the EU and USA for use in animal feed; in the US specifically for animals destined for human consumption. Its future use as a potential treatment in the fish food industry, therefore, is no longer considered.

\section{Toltrazuril}

The triazinetrione derivative coccidiostat toltrazuril has been shown to be effective against the protomont 
stage in in vitro trials (Schmahl et al. 1989; TojoRodriguez et al. 1994). However, when administrated in vivo it is either ineffective (Schmahl et al. 1989; Tojo-Rodriguez et al. 1994) or toxic to the fish (From et al. 1992).

THE POTENTIAL OF ALTERNATIVE CHEMICAL COMPOUNDS

Despite recent extensive research to explore the utility of alternative, environmentally friendly chemical compounds, only a handful of compounds have been shown to display efficacy at reducing I. multifiliis infections in vivo (see Table 1).

\section{In-bath treatments}

Of the bath compounds that have been identified, acetic acid (4\%), bronopol, peracetic acid-based products, combinations of peracetic acid and formaldehyde, humic acid (10\%) and potassium ferrate (VI) displayed a good level of efficacy. Acetic/ peracetic acid represents the cheapest treatment option, followed by, in rank order, formaldehyde, potassium ferrate (VI), and then significantly more expensive bronopol and humic acid, notably the latter. Of these compounds, acetic acid (4\%) is widely used in Turkey to control protozoan infections (Kayis et al. 2009). When tested in vivo against I. multifiliis, a single short dip bath of $10 \mathrm{ml}^{-1}$ for 3 min was able to reduce the trophont burden on treated fish (Balta et al. 2008).

Bronopol, the active compound of a product already licensed for use as an aquacultural drug, when applied at low concentrations (e.g. 2 and $5 \mathrm{mg} \mathrm{l}^{-1}$ ) over a long period of exposure (e.g. 27 days) was demonstrated to be highly effective against the free-swimming stages of $I$. multifiliis, as well as reducing the number of trophonts subsequently establishing in successive waves of infection (Shinn et al. 2011; Picón-Camacho et al. 2011a). Bronopol does not accumulate in fish tissues or in the environment and therefore no withdrawal period is required after its administration (Novartis, 2002). Bronopol presents no serious toxicological hazard to humans (Bryce et al. 1978) or to fish (Pottinger and Day, 1999), and, it degrades very quickly, especially when exposed to high intensity UV light (Noga, 2010). Bronopol-based products therefore show strong potential for the management of $I$. multifiliis infections in farm systems; however, timing of deployment with respect to parasite population dynamics and optimal treatment concentrations remain to be optimized for this product.

Formulations of peracetic acid (PAA), hydrogen peroxide and acetic acid have proven able to kill the protomont stage within $48 \mathrm{~h}$ of exposure at concentrations of $0 \cdot 8-0.9 \mathrm{mg}^{-1}$. Importantly, tomocysts recently attached to the substrate were also killed following a $12 \mathrm{~h}$ exposure to $1-3 \mathrm{mg} \mathrm{l}^{-1}$ to PAA solutions (Meinelt et al. 2009). When used in vivo, formulations containing a high proportion of PAA were also able to reduce the number of trophonts on infected fish (Rintamäki-Kinnunen et al. 2005a; Sudová et al. 2010). Adding peroctanoic acid to a PAA formulation, further improved the antiprotozoal activity of the solution, such that tomocyst stages were killed after $60 \mathrm{~min}$ exposure (Bruzio and Buchmann, 2010; Picón-Camacho et al. 2011b). PAA's stability, however, has been shown to be closely linked to a range of water quality parameters such as temperature, organic matter content and $\mathrm{pH}$ (Pedersen et al. 2009) and therefore the degradation of PAA must be assessed over time and taken into account in establishing the most effective treatment regime to use on site. The efficacy of PAA, notably against the tomocyst and trophont stages, however, highlights the potential of this compound as a treatment against $I$. multifiliis.

Low concentrations of humic acid (10\%) (100-150 $\left.\mu \mathrm{ll}^{-1}\right)$ were found to disrupt the development of protomonts; however, when the same concentrations were used in vivo, the results were inconsistent and appeared to be highly dependent on water temperature and the treatment regime used (Lahnsteiner and Weismann, 2007).

Ling et al. (2010) demonstrated that $4.8 \mathrm{mg} \mathrm{l}^{-1}$ potassium ferrate (VI) for $2 \mathrm{~h}$ was very effective in vitro, in killing theronts. When the same dose was used as an in vivo continuous bath treatment for 3 days, it resulted in an $80 \%$ reduction in the number of trophonts on the test fish. An increase in concentration to $19 \cdot 2 \mathrm{mg} \mathrm{l}^{-1}$ applied for 3 days managed to completely eradicate the infection from the fish stock suggesting that potassium ferrate (VI) is very successful at disrupting trophont development. Potassium ferrate (VI) is an environmentally friendly, strong oxidizing agent (Ma and Liu, 2002), that is less toxic to fish and humans than closely related potassium salts such as potassium permanganate (Ling et al. 2010). The effectiveness and degradation rate of potassium ferrate (VI) in the aquatic environment, however, is strongly linked to $\mathrm{pH}$ and water temperature (Johnson and Sharma, 1999) and these must be considered when establishing a treatment regime based on its use.

Of the bath chemicals that have investigated in recent years, potassium ferrate (VI), bronopol and the peracetic acid-based products all possess potential as promising alternatives to current chemotherapies for the control of $I$. multifiliis infections.

\section{In-feed treatments}

Of the in-feed treatments described in Table 1, the compounds with the highest apparent efficacy in vivo in controlling I. multifiliis infections are amprolium 
hydrochloride, vitamin C, quinine, SalarBec, salinomycim sodium and secnidazole. Shinn et al. (2003b) demonstrated that the two anti-coccidiostats compounds, amprolium hydrochloride and salinomycin sodium, when incorporated into a commercial feed, were able to significantly reduce the number of trophonts establishing on fish. Treatment with 100 $\mathrm{mg}^{-1}$ of amprolium hydrochloride (a thiamine, vitamin $\mathrm{B} 1$, analogue) for $1 \mathrm{~h}$ compromised the survival of the tomocyst stage in vitro, ultimately killing $85-90 \%$ of the tomocysts (Shinn et al. 2001). Incorporation of $1 \mathrm{~g} \mathrm{~kg}^{-1}$ of feed given over 8 days post-infection did not manage to reduce the trophont burden on fish (Tojo-Rodriguez et al. 1994). A dose $63 \mathrm{mg} \mathrm{kg}^{-1}$ of feed of amprolium hydrochloride given 10 days prior the infection, however, reduced the number of trophonts subsequently establishing on fish by up to $78 \%$ when compared to the control groups (Shinn et al. 2003b). Salinomycin sodium has only been tested in vivo, with promising results. Infected fish fed a diet containing $47-63 \mathrm{mg} \mathrm{kg}^{-1}$ of feed of salinomycin sodium for a period of 10 days were found to show a significant reduction (80-93\%) in number of trophonts when compared to the control groups (Shinn et al. 2003b). The same authors also tested SalarBec, a blend of Vitamin C, $\mathrm{E}$ and $\mathrm{B}$ group. When SalarBec was incorporated at a rate of $3 \cdot 2 \mathrm{~g} \mathrm{~kg}^{-1}$ feed and given to fish for a period of 10 days prior to infection with I. multifiliis, a $65 \%$ reduction in the number of trophonts surviving on challenged fish was found (Shinn et al. 2005).

Vitamin C on its own or in combination with Vitamin E has also been tested with success in vivo (Wahli et al. 1985, 1995, 1998). Quinine when incorporated into feed at a rate of $5 \mathrm{~g} \mathrm{~kg}^{-1}$ feed and given over a period of 7 to 10 days effected the complete elimination of $I$. multifiliis on medicated fish (Schmahl et al. 1996). Medicated fish using vitamin $\mathrm{C}$ and quinine, however, showed growth suppression as a result of decreased food intake.

Finally, secnidazole is an antibiotic which has been shown to reduce $I$. multifiliis infections when incorporated into feed and presented at $24-36 \mathrm{mg} \mathrm{kg}^{-1}$ of body weight (Tokşen and Nemli, 2010) or $40 \mathrm{~g} \mathrm{~kg}^{-1}$ of feed for 10 days (Tojo-Rodriguez and Santamarina-Fernandez, 2001). While secnidazole appeared to be effective, the cost of using it on a large commercial scale would be prohibitive (Noga, 2010).

Although the use of in-feed treatments appears to be an efficient, targeted strategy for reducing trophont burdens, the general inappetance displayed by heavily infected fish means that getting the target dose into infected fish in the later stages of an infection can be a challenge. This can, in part, be circumvented by top dressing unpalatable medicated diets (e.g. salinomycin sodium, see Shinn et al. $2003 b$ ) with bait flavouring to mask bitter ingredients and/or by incorporating feed stimulants (e.g. garlic) into the diet (Shinn unpublished data).

\section{NATURAL EXTRACTS}

Some new treatments involve the use of plant extracts such as those from garlic, Allium sativum L., which showed promising results when tested in vitro (Buchmann et al. 2003). However, when incorporated in-feed and tested in vivo this extract did not manage to significantly reduce infection levels when compared to control groups (Shinn et al. unpublished observations). Other natural products such as those from papaya Carica papaya L. and the velvet bean Mucuna pruriens L. were successful when tested in vitro and in vivo against protomonts and trophonts (Ekamen et al. 2004). Concentrations of 200 and $250 \mathrm{mg}^{-1}$ of C. papaya reduced the infection levels on treated fish by $89-92 \%$. M.pruriens administered at 100,150 and $200 \mathrm{mg} \mathrm{l}^{-1}$ also reduced the parasite burden on the treated fish by 59-92\%. Recent research by Yao et al. (2010) using the extract from Macleaya cordata Willd has shown high efficacy in in vitro trials against protomonts and an important trophont reduction (e.g. 75-97\%) when administered in vivo at low concentrations (e.g. $0 \cdot 6-0.9 \mathrm{mg} \mathrm{l}^{-1}$ ) for $48 \mathrm{~h}$. The use of probiotics as an in-feed treatment (e.g. $10^{8}$ cells of Aeromonas sobria $\mathrm{g}^{-1}$ feed for 14 days) has also proven to be very effective at reducing infections in medicated fish (Pieters et al. 2008).

There may therefore be considerable potential for the use of such natural products to control I. multifiliis infections; however, in vivo trials carried out under field conditions are a critical requirement prior to wider deployment of such treatments.

\section{NON-DRUG INTERVENTIONS}

In the last few years, a wide range of non-drug interventions (see Table 2) have been tested against I. multifiliis.

Farley and Heckmann (1980) used 'electrotherapy' as a possible treatment to control whitespot infections. Whilst there was some protomont mortality following exposure to short pulses of electricity $(5 \mathrm{sec})$, it seems that this was probably due to water hydrolysis rather than lysis of the parasite. It was concluded that the amperage necessary to disrupt trophonts within the fish epidermis would be too high and lethal to the fish.

The utilization of a single UV lamp $\left(91900 \mu \mathrm{W} \mathrm{s}^{-1} \mathrm{~cm}^{-2}\right)$ has, in contrast, successfully managed to reduce the mortality of fish infected with I. multifiliis in a closed re-circulation system by controlling the spread of I. multifiliis stages between tanks (Gratzek et al. 1983).

The mechanical filtration of inlet water, considering that the size of theronts ranges from 
$57 \cdot 4 \times 28.6 \mu \mathrm{m}\left(\right.$ at $5{ }^{\circ} \mathrm{C}$ ) and $28.6 \times 20 \cdot 0 \mu \mathrm{m}\left(\right.$ at $30^{\circ} \mathrm{C}$ ), is not a feasible method to prevent the entry of the parasite to farm systems (Aihua and Buchmann, 2001). Nonetheless, a combination of an $80 \mu \mathrm{m}$ mesh followed by a treatment of sodium percarbonate prevented protomonts from entering the system and killed theronts (Heinecke and Buchmann, 2009).

Bodensteiner et al. (2000) demonstrated that increasing the flow rate and water turnover in fish farms above $85 \mathrm{~cm} \mathrm{~min}^{-1}$ and $2 \cdot 1 \mathrm{l} \mathrm{h}^{-1}$ managed to reduce infection levels by flushing the free-swimming stages of the parasite out of the system. However, since water availability in farms can fluctuate greatly over the year, often reducing significantly over the summer months at the same time as water temperature increases exacerbate $I$. multifiliis infections, this cannot always provide a viable control solution.

Shinn et al. (2009) recently demonstrated that the combination of regular cleaning with a vacuum cleaning head and the use of a low adhesion polymer to line rainbow trout raceways is able to remove tomocysts and reduce infection levels by up to $99 \cdot 55 \%$ when compared to control groups. Notwithstanding their apparent efficacy, none of the management strategies described above have been adopted so far in a commercial fish farm context.

Despite these non-drug interventions, fish that are exposed to a certain level of I. multifiliis infection are able to acquire a protective immunity which can last from several months to a year (Hines and Spira, 1974; Burkart et al. 1990; Matthews, 1994). This acquired immunity has stimulated efforts towards the development of a vaccine against $I$. multifiliis which is in progress (Matthews, 2005; Sommerset et al. 2005; Dickerson, 2006).

\section{CONCLUSION}

Currently, the most frequent method employed to control I. multifiliis infections in farm systems is the use of in-bath chemical treatments. Because of its asynchronous life cycle and continuous release into the water column of different stages (Lom and Dyková, 1992; Matthews, 2005), multiple applications are often required over long periods of time, notably during the summer months when water temperatures can rise rapidly. In addition, outbreaks can occur in the spring and autumn seasons during which sharp changes in water temperature can induce physiological stress, as seen in channel catfish pond culture (Noga, 2010). Such treatment regimes involve the use of large quantities of chemicals when the infections levels are high (e.g. formaldehyde and sodium chloride), leading to high costs and potentially high environmental impacts. Repeated or prolonged use of a single drug without rotation of treatment types is also likely to increase the probability of development of drug resistance in the targeted pathogen, as documented for bacterial and copepod fish pathogens (Fallang et al. 2004; Lees et al. 2008; Heuer et al. 2009). While development of resistance by $I$. multifiliis has yet to be investigated, it is clear that drug resistance would act to increase the quantities of drug used and the environmental impacts of treatment.

In the present overview we have assessed the efficacy and practicality of a wide range of drug and non-drug strategies that are potentially available to be used in farm systems. However, there remain considerable difficulties in comparing efficacies between products, since no standardized methods are employed across the stakeholder community for culturing the parasite, assessing viability of the theront stage and infecting fish. The greatest current discrepancy in determining the efficacy of a treatment follows from the counting method employed for enumerating the trophont stage in in vivo studies. Some researchers only consider the trophonts present on skin scrapes or gills while others take into the account direct observations of the number of visible trophonts present in skin, fins and gills. In addition to these methodological variations, there is the fact that different strains/genotypes of $I$. multifiliis can behave very differently in terms of infectivity (Elsayed et al. 2006; Swennes et al. 2007; Ling et al. 2009), host specificity and susceptibility to treatment (Straus and Meinelt, 2009; Straus et al. 2009). Hence, a chemical treatment demonstrated to successfully eliminate one strain might not exhibit the same efficacy when applied to treat a different one.

From this review, chemical treatments remain the principal method for controlling I. multifiliis infections in aquaculture, despite numerous attempts to develop and implement physical and farm management-based alternatives. With the introduction of a ban on the use of malachite green in food-fish and a likely future ban on the use of formaldehyde, options for effective drug treatment remain severely depleted. For these reasons, considerable research has been conducted to develop new drugs or screen existing compounds, both natural and synthesized, for efficaciousness against one or more stages of this parasite. New products, where deployed, will need to be derived from sustainable sources and of themselves be more environmentally friendly and more suitable for use in food-fish than previous compounds. As part of the attempt to reduce the use of drugs, new deployment strategies (e.g. extended lowdose treatments), management strategies helping to reduce initial infection levels (e.g. flow control), breeding fish for resistance and the development of DNA vaccines need to be considered.

\section{FINANCIAL SUPPORT}

Part of this study was supported by the British Federation of Women in Science (BFWS) awarded to Sara Picón Camacho to support her Ph.D. project. 
Table 1. Chemical treatments tested against infections of Ichthyophthirius multifiliis Fouquet, 1876

(A compound is regarded as being partially effective if it kills $50-80 \%$, and effective if it kills $\geqslant 80 \%$ of the stages under test. Mortality refers to the parasite stages unless otherwise stated.)

\begin{tabular}{|c|c|c|c|c|}
\hline Compound & Dose & $\begin{array}{l}\text { Host/parasite } \\
\text { stage }\end{array}$ & Efficacy & Reference \\
\hline \multicolumn{5}{|c|}{ Acaprin (1, 3-di-6-quinolylurea) } \\
\hline In vitro & $200 \mathrm{mg} \mathrm{l}^{-1}$ for $2 \mathrm{~h}$ & Protomonts & Partially effective $-62 \cdot 5 \%$ mortality after $1-2 \mathrm{~h}$ & $\begin{array}{l}\text { Tojo-Rodriguez et al. } \\
\text { (1994) }\end{array}$ \\
\hline In vivo-bath & $200 \mathrm{mg}^{-1}$ for $3 \mathrm{~h}$ & Oncorhynchus & $\begin{array}{l}60 \% \text { of surviving protomonts develop normally } \\
\text { Not effective - all trophonts developed normally } \dagger\end{array}$ & $\begin{array}{l}\text { Tojo-Rodriguez et al. } \\
\text { (1994) }\end{array}$ \\
\hline In-feed & $500 \mathrm{mg} \mathrm{kg}^{-1}$ for $8 \mathrm{~d}$ & mykiss & Not effective - all trophonts developed normally $\dagger$ & $\begin{array}{l}\text { Tojo-Rodriguez and } \\
\text { Santamarina- } \\
\text { Fernandez }(2001)\end{array}$ \\
\hline
\end{tabular}

Acetic acid (4\%)

In vivo-bath

$10 \mathrm{ml} \mathrm{l}^{-1}$ for $3 \mathrm{~min}$

O. mykiss

Salvelinus fontinalis

Salmo trutta

Allium sativum (garlic extract)

$0 \cdot 5 \mathrm{mg}^{-1}$ for $1 \cdot 5,3$ and $15 \mathrm{~h}$

$2 \cdot 5 \mathrm{mg} \mathrm{l}^{-1}$ for $1 \cdot 5,3$ and $15 \mathrm{~h}$

$12 \cdot 5 \mathrm{mg} \mathrm{l}^{-1}$ for $1 \cdot 5,3$ and $15 \mathrm{~h}$

$62.5 \mathrm{mg} \mathrm{l}^{-1}$ for 1.5 and $3 \mathrm{~h}$

$62 \cdot 5 \mathrm{mg} \mathrm{l}^{-1}$ for $15 \mathrm{~h}$

$312.5 \mathrm{mg} \mathrm{l}^{-1}$ for $1.5 \mathrm{~h}$

$312.5 \mathrm{mg} \mathrm{l}^{-1}$ for 3 and $15 \mathrm{~h}$

$1562 \cdot 5 \mathrm{mg} \mathrm{l}^{-1}$ for 3 and $15 \mathrm{~h}$

$30 \mathrm{mg} \mathrm{l}^{-1}$ for $24 \mathrm{~h}$

$117 \mathrm{mg} \mathrm{l}^{-1}$ for $24 \mathrm{~h}$

$570 \mathrm{mg} \mathrm{l}^{-1}$ for $24 \mathrm{~h}$

Amphotericin B (dissolved in Na-desoxycholate)

In vitro $\quad 0.25 \mathrm{mg} \mathrm{l}^{-1}$ for $24 \mathrm{~h}$

$2.5 \mathrm{mg} \mathrm{l}^{-1}$ for $24 \mathrm{~h}$

$0 \cdot 25 \mathrm{mg} \mathrm{l}^{-1}$ for $24 \mathrm{~h}$

$2 \cdot 5 \mathrm{mgl}^{-1}$ for $24 \mathrm{~h}$

$0 \cdot 25 \mathrm{mg} \mathrm{l}^{-1}$ for $3 \mathrm{~h}$

$2 \cdot 5 \mathrm{mg} \mathrm{l}^{-1}$ for $3 \mathrm{~h}$

$0 \cdot 25 \mathrm{mg} 1^{-1}-2 \times$ for $1 \mathrm{~h}$

1 st: day 9 p.i, 2 nd: day 12 p.i

Theronts

Partially effective - reduction of the number of

trophonts on treated fish but no details given

Balta et al. (2008)

Not effective $-<50 \%$ mortality

Not effective $-<50 \%$ mortality

Not effective $-<50 \%$ mortality

Not effective $-<50 \%$ mortality

Partially effective $->50 \%$ mortality

Not effective $-<50 \%$ mortality

Partially effective $->50 \%$ mortality

Partially effective $->50 \%$ mortality

Tomocysts

Not effective $-13 \%$ mortality

Partially effective $-53 \%$ mortality

Effective $-100 \%$ mortality

Adults**

Effective $-100 \%$ mortality after $24 \mathrm{~h}$

Effective $-100 \%$ mortality after $1 \mathrm{~h}$

Tomocysts

Effective $-100 \%$ mortality after $24 \mathrm{~h}$

Effective $-100 \%$ mortality after $24 \mathrm{~h}$

Effective $-100 \%$ mortality after $1 \mathrm{~h}$.

Effective $-100 \%$ mortality after $5 \mathrm{~min}$

O. mykiss

Not effective - no details

Wahli et al. (1993)

Buchmann et al. (2003)

Wahli et al. (1993)

Amprolium hydrochloride (1-[(4-amino-2-propyl-5-pyrimidinyl) methyl]-2-picolinium chloride hydrochloride) commercialised as Amprolmix

In vitro $\quad 20 \mathrm{mg} \mathrm{l}^{-1}$ for $1 \mathrm{~h} \quad$ Protomonts

Not effective $-10 \%$ mortality

Shinn et al.

(unpublished) 


\begin{tabular}{|c|c|c|}
\hline & $\begin{array}{l}100 \mathrm{mg} \mathrm{l}^{-1} \text { for } 1 \mathrm{~h} \\
200 \mathrm{mgl}^{-1} \text { for } 2 \mathrm{~h}\end{array}$ & Protomonts \\
\hline & $1000 \mathrm{mg}^{-1}$ for $48 \mathrm{~h}$ & \\
\hline & $\begin{array}{l}100 \mathrm{mg} \mathrm{l}^{-1} \text { for } 15 \mathrm{~h} \\
100 \mathrm{mg} \mathrm{l}^{-1} \text { for } 41 \mathrm{~h}\end{array}$ & Tomocysts \\
\hline & $20 \mathrm{mg} \mathrm{l}^{-1}$ for $1 \mathrm{~h}$ & Theronts \\
\hline & $\begin{array}{l}50 \mathrm{mg} \mathrm{l}^{-1} \text { for } 1 \mathrm{~h} \\
100 \mathrm{mg} \mathrm{l}^{-1} \text { for } 1 \mathrm{~h}\end{array}$ & \\
\hline In vivo-bath & $200 \mathrm{mg} \mathrm{l}^{-1}$ for $3 \mathrm{~h}$ day 6 p.i. & O. mykiss \\
\hline In-feed & $63 \mathrm{mg} \mathrm{kg}^{-1}$ feed for $10 \mathrm{~d}$ prior inf. & O. mykiss \\
\hline & $\begin{array}{l}75 \mathrm{mg} \mathrm{kg}^{-1} \text { feed for } 10 \mathrm{~d} \mathrm{p.i.} \\
75 \mathrm{mg} \mathrm{kg}^{-1} \text { feed for } 10 \mathrm{~d} \text { prior inf. }\end{array}$ & \\
\hline & $104 \mathrm{mg} \mathrm{kg}^{-1}$ feed for $10 \mathrm{~d}$ p.i. & \\
\hline & $1000 \mathrm{mg} \mathrm{kg}^{-1}$ feed for $8 \mathrm{~d}$ p.i. & O. mykiss \\
\hline
\end{tabular}

Amprolmix

See entry for amprolium hydrochloride

Aeromonas sobria
In vivo - In-feed
$10^{8}$ cells $\mathrm{g}^{-1}$ feed for $14 \mathrm{~d}$
O. mykiss

Aquahumin

See entry for humic acid (10\% solution)

Ascorbate-2-phosphate (vitamin C)
In vivo-In-feed
$5000 \mathrm{mg} \mathrm{kg}^{-1}$ feed for $9 \mathrm{~d}$
$50 \mathrm{mg} 200 \mathrm{~kg}^{-1}$ feed
O. mykiss

$50 \mathrm{mg} 2000 \mathrm{~kg}^{-1}$ feed

1-3 $\mathrm{g} \mathrm{kg}^{-1}$ feed for 1 week-1 month

Not specified

Ascorbate-2-phosphate (Vitamin C) +d-1-alpha-tocopheryl acetate (Vitamin E) complex diet In vivo - In-feed for 7 weeks

$$
\begin{aligned}
& 0+1 \cdot 8 \mathrm{mg} \mathrm{kg}^{-1} \text { feed } \\
& 4 \cdot 3+771 \cdot 0 \mathrm{mg} \mathrm{kg}^{-1} \text { feed } \\
& 24 \cdot 8+34 \cdot 0 \mathrm{mg} \mathrm{kg}^{-1} \text { feed } \\
& 27 \cdot 5+776 \cdot 0 \mathrm{mg} \mathrm{kg}^{-1} \text { feed } \\
& 2065 \cdot 0+2 \cdot 5 \mathrm{mg} \mathrm{kg}^{-1} \text { feed } \\
& 2093 \cdot 3+30 \cdot 8 \mathrm{mg} \mathrm{kg}^{-1} \text { feed } \\
& 2025 \cdot 0+754 \cdot 3 \mathrm{mg} \mathrm{kg}^{-1} \text { feed }
\end{aligned}
$$

Not effective $-90 \%$ mortality

Not effective $-0 \%$ mortality after $2 \mathrm{~h}$; prototomonts Tojo-Rodriguez et al.

developed normally

Not effective-survival not affected

Effective $-85 \%$ mortality

Effective $-90 \%$ mortality

Not effective $-22 \cdot 4 \%$ mortality

Not effective $-20 \cdot 4 \%$ mortality

Not effective $-22 \cdot 3 \%$ mortality

Not effective - all trophonts developed normally

Partially effective $-77 \cdot 6 \%$ reduction in trophont numbers

Not effective $-32 \cdot 2 \%$ reduction in trophont numbers

Partially effective $-63 \%$ reduction in trophont numbers

Partially effective $-62 \%$ reduction in trophont numbers

Not effective - all trophonts developed normally$$
\text { (1994) }
$$

Farley and Heckmann

(1980)

Shinn et al. (2001)

Shinn et al.

(unpublished)

Shinn et al. (2001)

Tojo-Rodriguez et al.

$$
\text { (1994) }
$$

Shinn et al. $(2003 b)$

Shinn et al. (2001)

Shinn et al. (2003b)

Tojo-Rodriguez et al. (1994)

Pieters et al. (2008)

Effective - there was no mortality of treated fish

Partially effective but $2-16 \%$ of medicated fish died Partially effective - reduction in trophont numbers but no details

Partially effective - reduction in trophont numbers

but no details

Effective - no detail

Wahli et al. (1985)

Wahli et al. (1995)

Rahkonen and Koski (2002)

Not effective $-44 \%$ of medicated fish died 'Effective' but $\sim 20 \%$ of medicated fish died Not effective $-62 \%$ of medicated fish died 'Effective' but $\sim 20 \%$ of medicated fish died

'Effective' but $<20 \%$ of medicated fish died

'Effective' but $\sim 20 \%$ of medicated fish died 'Effective' but $<20 \%$ of medicated fish died

Walhi et al. (1998) 


\begin{tabular}{|c|c|c|c|c|}
\hline Compound & Dose & $\begin{array}{l}\text { Host/parasite } \\
\text { stage }\end{array}$ & Efficacy & Reference \\
\hline $\begin{array}{l}\text { Ascorbyl phosphate } \\
\text { In vivo-In-feed }\end{array}$ & $50 \mathrm{mg} 2000 \mathrm{~kg}^{-1}$ feed & O. mykiss & $\begin{array}{l}\text { Partially effective - reduction in trophont numbers } \\
\text { but no details }\end{array}$ & Wahli et al. (1995) \\
\hline \multicolumn{5}{|c|}{$\begin{array}{l}\text { Baycox } \\
\text { See entry for toltrazuril }\end{array}$} \\
\hline $\begin{array}{l}\text { Bithionol } \\
\text { In vivo-In feed }\end{array}$ & $40 \mathrm{~g} \mathrm{~kg}^{-1}$ of feed for $10 \mathrm{~d}$ & O. mykiss & $\begin{array}{l}\text { Not effective }-68 \% \text { fish with high number of } \\
\text { trophonts }\end{array}$ & $\begin{array}{l}\text { Tojo-Rodriguez and } \\
\text { Santamarina-Fernandez } \\
(2001)\end{array}$ \\
\hline \multirow[t]{3}{*}{ In vitro } & $\begin{array}{l}\text { 2-nitropropane- } 1,3 \text {-diol) } \\
20 \mathrm{mg}^{-1} \text { for } 30 \mathrm{~min} \\
50 \mathrm{mg}^{-1} \text { for } 30 \mathrm{~min} \\
100 \mathrm{mg}^{-1} \text { for } 30 \mathrm{~min}\end{array}$ & Protomonts & $\begin{array}{l}\text { Partially effective }-76 \cdot 2 \% \text { mortality } \\
\text { Effective }-97 \cdot 2 \% \text { mortality } \\
\text { Effective }-100 \% \text { mortality }\end{array}$ & Shinn et al. (in press) \\
\hline & $\begin{array}{l}20 \mathrm{mg} \mathrm{l}^{-1} \text { for } 30 \mathrm{~min} \\
50 \mathrm{mg} \mathrm{l}^{-1} \text { for } 30 \mathrm{~min}\end{array}$ & Tomocysts & $\begin{array}{l}\text { Not effective }-3 \cdot 3 \% \text { mortality; tomocyst } \\
\text { development delayed } \\
\text { Not effective }-10 \% \text { mortality; tomocyst development } \\
\text { delayed }\end{array}$ & Shinn et al. (in press) \\
\hline & $\begin{array}{l}0 \cdot 1 \mathrm{mg} \mathrm{l}^{-1} \text { for } 12 \mathrm{~h} \\
0 \cdot 1 \mathrm{mg} \mathrm{l}^{-1} \text { for } 24 \mathrm{~h} \\
0 \cdot 1 \mathrm{mg} \mathrm{l}^{-1} \text { for } 36 \mathrm{~h} \\
0 \cdot 1 \mathrm{mg} \mathrm{l}^{-1} \text { for } 48 \mathrm{~h} \\
0 \cdot 25 \mathrm{mg} \mathrm{l}^{-1} \text { for } 12 \mathrm{~h} \\
0 \cdot 25 \mathrm{mg} \mathrm{l}^{-1} \text { for } 24 \mathrm{~h} \\
0 \cdot 25 \mathrm{mg} \mathrm{l}^{-1} \text { for } 36 \mathrm{~h} \\
0 \cdot 25 \mathrm{mg} \mathrm{l}^{-1} \text { for } 48 \mathrm{~h} \\
0 \cdot 5 \mathrm{mg} \mathrm{l}^{-1} \text { for } 12 \mathrm{~h} \\
0 \cdot 5 \mathrm{mg} \mathrm{l}^{-1} \text { for } 24 \mathrm{~h} \\
0 \cdot 5 \mathrm{mg} \mathrm{l}^{-1} \text { for } 36 \mathrm{~h} \\
0 \cdot 5 \mathrm{mg} \mathrm{l}^{-1} \text { for } 48 \mathrm{~h} \\
0 \cdot 75 \mathrm{mg} \mathrm{l}^{-1} \text { for } 12 \mathrm{~h} \\
0 \cdot 75 \mathrm{mg} \mathrm{l}^{-1} \text { for } 24 \mathrm{~h} \\
0 \cdot 75 \mathrm{mg} \mathrm{l}^{-1} \text { for } 36 \mathrm{~h} \\
0 \cdot 75 \mathrm{mg}^{-1} \text { for } 48 \mathrm{~h} \\
1 \mathrm{mg} \mathrm{l^{-1 }} \text { for } 12 \mathrm{~h} \\
1 \mathrm{mg} \mathrm{l}^{-1} \text { for } 24 \mathrm{~h} \\
1 \mathrm{mg} \mathrm{l}^{-1} \text { for } 36 \mathrm{~h} \\
1 \mathrm{mg} \mathrm{l}^{-1} \text { for } 48 \mathrm{~h} \\
1 \mathrm{mg} \mathrm{l^{-1 }} \text { for } 12 \mathrm{~h} \\
1 \mathrm{mg} \mathrm{l}^{-1} \text { for } 24 \mathrm{~h} \\
20 \mathrm{mg} \mathrm{l}^{-1} \text { for } 30 \mathrm{~min}\end{array}$ & Theronts & $\begin{array}{l}\text { Not effective }-7 \cdot 15 \% \text { mortality } \\
\text { Not effective }-31 \cdot 55 \% \text { mortality } \\
\text { Not effective }-31 \cdot 66 \% \text { mortality } \\
\text { Not effective }-18 \% \text { mortality } \\
\text { Not effective }-14 \cdot 03 \% \text { mortality } \\
\text { Not effective }-30 \cdot 95 \% \text { mortality } \\
\text { Not effective }-40 \cdot 0 \% \text { mortality } \\
\text { Not effective }-34 \cdot 84 \% \text { mortality } \\
\text { Not effective }-22 \cdot 38 \% \text { mortality } \\
\text { Not effective }-38 \cdot 77 \% \text { mortality } \\
\text { Not effective }-26 \cdot 51 \% \text { mortality } \\
\text { Partially effective }-59 \cdot 21 \% \text { mortality } \\
\text { Not effective }-8 \cdot 88 \% \text { mortality } \\
\text { Not effective }-40 \cdot 0 \% \text { mortality } \\
\text { Not effective }-26 \cdot 51 \% \text { mortality } \\
\text { Partially effective }-68 \cdot 57 \% \text { mortality } \\
\text { Not effective }-13 \cdot 88 \% \text { mortality } \\
\text { Not effective }-37 \cdot 93 \% \text { mortality } \\
\text { Not effective }-44 \cdot 44 \% \text { mortality } \\
\text { Partially effective }-75 \cdot 0 \% \text { mortality } \\
\text { Partially effective }-70 \cdot 84 \% \text { mortality } \\
\text { Effective }-100 \% \text { mortality } \\
\text { Not effective }-18 \cdot 5 \% \text { mortality }\end{array}$ & Shinn et al. (in press) \\
\hline
\end{tabular}


$0 \mathrm{mg} \mathrm{l}^{-1}$ for $30 \mathrm{~min}$

$100 \mathrm{mg} \mathrm{l}^{-1}$ for $30 \mathrm{~min}$

$100 \mathrm{mg} \mathrm{l}^{-1}$ for $30 \mathrm{~min}$

In vivo-bath

$1 \mathrm{mg} \mathrm{l}^{-1}$ for $36 \mathrm{~d} \mathrm{p.i}$

$2 \mathrm{mg} \mathrm{l}^{-1}$ for $36 \mathrm{~d} \mathrm{p}$.

$2 \mathrm{mg} \mathrm{l}^{-1} 24 \mathrm{~h}$ prior inf. and $72 \mathrm{~h} \mathrm{p.i.}$

$5 \mathrm{mg} \mathrm{l}^{-1}$ for $36 \mathrm{~d} \mathrm{p.i}$

$50 \mathrm{mg} \mathrm{l}^{-1}$ daily for $1 \mathrm{~h}$ for $10 \mathrm{~d}$

O. mykiss

$50 \mathrm{mg} \mathrm{l}^{-1}$ for $30 \mathrm{~min}$ for $10 \mathrm{~d}$

(alternate days)

$100 \mathrm{mg}^{-1}$ daily for $30 \mathrm{~min}$ for $10 \mathrm{~d}$

$100 \mathrm{mg} \mathrm{l}^{-1}$ daily for $30 \mathrm{~min}$ for $10 \mathrm{~d}$

$100 \mathrm{mg} \mathrm{l}^{-1}$ for $1 \mathrm{~h}$ on day 7

Brochothrix thermosphacto

In vivo-In-feed $10^{10}$ cells g ${ }^{-1}$ feed for $14 \mathrm{~d}$

O. mykiss

0.005 ppm for $18-22 \mathrm{~h}$

$0.05 \mathrm{ppm}$ for $10 \mathrm{~min}, 1$ and $5 \mathrm{~h}$

$0.05 \mathrm{ppm}$ for $18-22 \mathrm{~h}$

$0.5 \mathrm{ppm}$ for $10 \mathrm{~min}$ and $1 \mathrm{~h}$

$0 \cdot 5 \mathrm{ppm}$ for $5 \mathrm{~h}$

$0.5 \mathrm{ppm}$ for $18-22 \mathrm{~h}$

5 ppm for $10 \mathrm{~min}$

5 ppm for $1 \mathrm{~h}$

$5 \mathrm{ppm}$ for $5 \mathrm{~h}$

$50 \mathrm{ppm}$ for $10 \mathrm{~min}$

$50 \mathrm{ppm}$ for $1 \mathrm{~h}$

$500 \mathrm{ppm}$ for $10 \mathrm{~min}$

Carica papaya (papaya)

In vitro

$100 \mathrm{mg} \mathrm{l}^{-1}$ for $3 \mathrm{~h}$

$100 \mathrm{mg} \mathrm{l}^{-1}$ for $6 \mathrm{~h}$

$150 \mathrm{mg} \mathrm{l}^{-1}$ for $3 \mathrm{~h}$

$150 \mathrm{mg} \mathrm{l}^{-1}$ for $6 \mathrm{~h}$

$200 \mathrm{mg} \mathrm{l}^{-1}$ for $3 \mathrm{~h}$

$200 \mathrm{mg} \mathrm{l}^{-1}$ for $6 \mathrm{~h}$

$250 \mathrm{mg} \mathrm{l}^{-1}$ for $3 \mathrm{~h}$

O. mykiss
Not effective $-31 \cdot 3 \%$ mortality

Partially effective $-51 \cdot 7 \%$ mortality

Effective $-50 \%$ mortality; all dead after $43 \mathrm{~h}$

Shinn et al. (in press)

Not effective - number of trophonts increased on

treated group

Effective $-46 \%$ reduction in trophont numbers on the

2 nd wave of infection; $83 \%$ reduction in trophonts

numbers on 3 rd wave

Partially effective $-35-48 \%$ trophont reduction on

treated groups of infection; $97 \%$ reduction in

trophont numbers on 3 rd wave

Effective $-83 \%$ reduction in trophont numbers on the 2nd wave

Not effective - no reduction in trophont numbers on Shinn et al. (2003a)

treated fish

Not effective - no significant reduction in trophont numbers

Picón-Camacho et al.

(in press $a$ )

Not effective $-33 \cdot 3 \%$ reduction in trophont number

Effective $-81 \cdot 1 \%$ reduction in trophont numbers

Not effective - no reduction in trophont numbers on

treated fish

Not effective $-98 \%$ mortality on treated fish

Not effective $-0 \%$ mortality

Not effective $-0 \%$ mortality

Effective $-50-90 \%$ mortalit

Not effective $-0 \%$ mortality

Effective - 50-90\% mortality

Effective $-100 \%$ mortality

Not effective - reduction in swimming velocity

Effective - 50-90\% mortality

Effective $-100 \%$ mortality

Not effective-reduction in swimming velocity

Effective $-100 \%$ mortality

Effective $-100 \%$ mortality

Trophonts*

Not effective $-0 \%$ mortality

Not effective $-10 \%$ mortality

Not effective $-5 \%$ mortality

Partially effective $-55 \%$ mortality

Not effective $-25 \%$ mortality

Effective $-100 \%$ mortalit

Effective $-90 \%$ mortality
Pieters et al. (2008)

Shinn et al.

(unpublished)

Shinn et al. (2003a)

Bisharyan et al. (2003)

Ekamen et al. (2004) 


\begin{tabular}{|c|c|c|}
\hline Compound & Dose & $\begin{array}{l}\text { Host/parasite } \\
\text { stage }\end{array}$ \\
\hline In vivo-bath & $\begin{array}{l}250 \mathrm{mg} \mathrm{l}^{-1} \text { for } 6 \mathrm{~h} \\
200 \mathrm{mg} \mathrm{l}^{-1} \text { for } 92 \mathrm{~h} \\
250 \mathrm{mg} \mathrm{l}^{-1} \text { for } 92 \mathrm{~h}\end{array}$ & Carassius $a . a$ \\
\hline $\begin{array}{l}\text { Chloramine- } \mathrm{T}(\mathrm{s} \\
\text { In vitro }\end{array}$ & $\begin{array}{l}\mathrm{m} \mathrm{p} \text {-toluenesulfonchloramide) } \\
5 \mathrm{mg} 1^{-1} \text { for } 1 \mathrm{~h} \\
50 \mathrm{mg} \mathrm{l}^{-1} \text { for } 15 \mathrm{~min}\end{array}$ & $\begin{array}{l}\text { Protomonts } \\
\text { Theronts }\end{array}$ \\
\hline In vivo-bath & $\begin{array}{l}1 \mathrm{mg} \mathrm{l}^{-1} \text { daily for } 11 \mathrm{~d} \\
2 \mathrm{mg} \mathrm{l}^{-1} \text { daily for } 11 \mathrm{~d} \\
5 \mathrm{mg} \mathrm{l}^{-1} \text { daily for } 11 \mathrm{~d} \\
5,10 \text { and } 15 \mathrm{mg} \mathrm{l}^{-1} \text { for } 1 \mathrm{~h}\end{array}$ & $\begin{array}{l}\text { O. mykiss } \\
\text { S. fontinalis } \\
\text { S.trutta }\end{array}$ \\
\hline & $10 \mathrm{mg} \mathrm{l}^{-1}$ for $6 \mathrm{~h}$ & O. mykiss \\
\hline & $\begin{array}{l}14 \mathrm{mg} \mathrm{l}^{-1} 3 \text { times a week for } 3 \text { weeks } \\
16 \mathrm{mg} \mathrm{l}^{-1} 3 \text { times a week for } 2 \text { weeks }\end{array}$ & Salmo salar \\
\hline & $\begin{array}{l}100 \mathrm{mg} \mathrm{l}^{-1} \text { for } 30 \mathrm{~min} \text { daily over } \\
10 \mathrm{~d} \text { period }^{\text {. }}\end{array}$ & O. mykiss \\
\hline & $\begin{array}{l}100 \mathrm{mg} \mathrm{l}^{-1} \text { for } 1 \mathrm{~h} \text { on day } 7 \mathrm{p} . \mathrm{i} . \\
100 \mathrm{mg} \mathrm{l}^{-1} \text { for } 6 \mathrm{~h} \text { on day } 7 \mathrm{p} . \mathrm{i} . \\
100 \mathrm{mg} \mathrm{l}^{-1} \text { daily for } 30 \mathrm{~min} \text { for } 10 \mathrm{~d} \\
100 \mathrm{mg} \mathrm{l}^{-1} \text { daily for } 30 \mathrm{~min} \text { for } 10 \mathrm{~d} \\
100 \mathrm{mg} \mathrm{l}^{-1} \text { for } 30 \mathrm{~min} 4 \text { times over } 10 \mathrm{~d}\end{array}$ & O. mykiss \\
\hline
\end{tabular}

Chloramine- $\mathrm{T}$ (sodium p-toluenesulfonchloramide) + formaldehyde

In vivo - bath $\quad 10+100 \mathrm{mg} \mathrm{l}^{-1} 3$ times a week for $\quad$ S. salar 4 weeks $8+125 \mathrm{mg} \mathrm{l}^{-1} 3$ times a week for 5 weeks

Chloramphenicol (D (-) threo-2,2-dichloro-N-[hydroxy- $\alpha$ (hydroxymethyl)-p-nitrophenethyl] acetamide)

In vitro

$\begin{array}{lll}100 \mathrm{mg} \mathrm{l}^{-1} \text { for } 3 \mathrm{~h} & \text { Theronts } & \text { Not effective }- \text { no details } \\ 100 \mathrm{mg} \mathrm{l}^{-1} \text { for } 24 \mathrm{~h} & \text { Adults** } & \text { Not effective }- \text { no details } \\ 100 \mathrm{mg}^{-1} \text { for } 24 \mathrm{~h} & \text { Tomocysts } & \text { Not effective }- \text { no details }\end{array}$

Reference

Effective $-100 \%$ mortality

Effective $-89 \%$ reduction in trophont number on the Ekamen et al. (2004) skin and fins

Effective $-92 \%$ reduction in trophont number on the skin and fins

Effective $-100 \%$ mortality

Effective $-100 \%$ mortality

Not effective $-100 \%$ mortality on treated fish

Not effective $-100 \%$ mortality on treated fish

Not effective $-100 \%$ mortality on treated fish

Not effective - no details

Shinn et al. (2001)

Tieman and Goodwin (2001)

Balta et al. (2008)

Not effective - no reduction in trophont numbers

Shinn et al.

(unpublished)

Not effective - parasite numbers increased over infection period

Trial inconclusive - low parasite numbers across all

$$
\text { groups }
$$

Effective $-93 \%$ reduction in trophont numbers

Not effective $-14 \%$ reduction in trophont numbers

Not effective - no reduction in trophont numbers

Effective $-90 \cdot 5 \%$ reduction in trophont numbers

Effective $-97 \cdot 3 \%$ reduction in trophont numbers

Partially effective - significant reduction in $50 \%$ of the Rahkonen and Koski tanks

\section{(2002)}

Rintamäki-Kinnunen et al. $(2005 a)$

Rintamäki-Kinnunen

et al. $(2005 b)$

Wahli et al. (1993) 


\section{Chloroquine}

In vitro

$200 \mathrm{mg} \mathrm{l}^{-1}$ for $2 \mathrm{~h}$

Protomonts

O. mykiss

In vivo-bath

$200 \mathrm{mg} \mathrm{l}^{-1}$ for $3 \mathrm{~h}$

$1000 \mathrm{mg} \mathrm{kg}^{-1}$ feed for $8 \mathrm{~d}$

$40 \mathrm{~g} \mathrm{~kg}^{-1}$ feed for $10 \mathrm{~d}$

O. mykiss

Chlortetracycline

In vitro

$100 \mathrm{mg} \mathrm{l}^{-1}$ for $24 \mathrm{~h}$

$100 \mathrm{mg} \mathrm{l}^{-1}$ for $3 \mathrm{~h}$

Adults**

Theronts

$100 \mathrm{mg} \mathrm{l}^{-1}$ for $24 \mathrm{~h}$

Tomocysts

In vivo-bath

$100 \mathrm{mg} \mathrm{l}^{-1}-2 \times$ for 1

O. mykiss

In-feed

st: day 9 p.i, 2 nd: day 12 p.i

Citrocid

In vivo-In-feed $10 \mathrm{mg} \mathrm{kg}^{-1}$ feed for $7 \mathrm{~d} \mathrm{p.i.}$

O. mykiss

Citrox BC

In vivo - In-feed $10 \mathrm{mg} \mathrm{kg}^{-1}$ feed for $7 \mathrm{~d}$ p.i

O. mykiss

Clopidol (3,5-dichloro-2,6-dimethyl-4-pyridinol) commercialised as Coyden

In vivo-In-feed $65 \mathrm{mg} \mathrm{kg}^{-1}$ feed for $10 \mathrm{~d}$ prior inf.

$92 \mathrm{mg} \mathrm{kg}^{-1}$ feed for $10 \mathrm{~d}$ prior inf.

$72 \mathrm{mg} \mathrm{kg}^{-1}$ feed for $10 \mathrm{~d}$ prior inf.

Copper sulphate $\left(\mathrm{CuSO}_{4}\right)$

In vitro

$55 \mu \mathrm{g} \mathrm{l}^{-1}$ for $24 \mathrm{~h}$

$110 \mu \mathrm{g} \mathrm{l}^{-1}$ for $24 \mathrm{~h}$

$160 \mu \mathrm{g} \mathrm{l}^{-1}$ for $24 \mathrm{~h}$

$255 \mu \mathrm{g} \mathrm{l}^{-1}$ for $24 \mathrm{~h}$

$220 \mu \mathrm{g} \mathrm{l}^{-1}$ for $24 \mathrm{~h}$

$0.027 \mathrm{mg} \mathrm{Cu}^{-1}$ as $\mathrm{CuSO}_{4}$ for $3 \mathrm{~h}$

(alkalinity $48 \mathrm{mg} \mathrm{l}^{-1}$ )

$0 \cdot 028 \mathrm{mg}$ nonchelated liquid $\mathrm{CuSO}_{4} \mathrm{I}^{-1}$

for $3 \mathrm{~h}$ (alkalinity $48 \mathrm{mg} \mathrm{l}^{-1}$ )

$0.027 \mathrm{mg} \mathrm{Cu} \mathrm{l} l^{-1}$ as $\mathrm{CuSO}_{4}$ for $3 \mathrm{~h}$

(alkalinity $48 \mathrm{mg} \mathrm{l}^{-1}$ )

$0 \cdot 05 \mathrm{mg} \mathrm{Cu} \mathrm{l}^{-1}$ as $\mathrm{CuSO}_{4}$ for $3 \mathrm{~h}$

(alkalinity $48 \mathrm{mg} \mathrm{l}^{-1}$ )

$0 \cdot 05 \mathrm{mg}$ nonchelated liquid $\mathrm{CuSO}_{4} \mathrm{1}^{-1}$

for $2 \mathrm{~h}$ (alkalinity $48 \mathrm{mg} \mathrm{l}^{-1}$ )
Partially effective $-50 \%$ mortality after $2 \mathrm{~h}$.

Tojo-Rodriguez et al (1994)

Protomonts surviving develop normally

Not effective - all trophonts developed normally

Not effective - all trophonts developed normally

Not effective-high numbers of trophonts on al

treated fish

Effective $-100 \%$ mortality after $1 \mathrm{~h}$

Effective $-100 \%$ mortality after 5 min

Effective $-100 \%$ mortality after $24 \mathrm{~h}$

Not effective - no details

Tojo-Rodriguez et al (1994)

Tojo-Rodriguez and

Santamarina-Fernandez (2001)

Wahli et al. (1993)

Wahli et al. (1993)

Not effective - no details

Not effective $\sim 40 \%$ reduction in trophont numbers

Shinn et al. (2005)

Not effective $-25 \%$ reduction in trophont numbers

Shinn et al. (2005)

Not effective $-35 \cdot 2 \%$ reduction in trophont number

Not effective $-20 \cdot 1 \%$ reduction in trophont number

Not effective $-35.6 \%$ reduction in trophont numbers

Not effective $-100 \%$ manage to infect $C$. auratus

Not effective $-90 \%$ manage to infect $C$. auratus

Effective-tomites inactive but $20 \%$ manage to infect

C. auratus

Effective $-100 \%$ mortality

Effective $-100 \%$ mortality

Partially effective $-50 \%$ mortality

Goodwin and Straus

Partially effective $-50 \%$ mortality

Partially effective $-50 \%$ mortality

Effective $-95 \%$ mortality

Effective $-95 \%$ mortality
Shinn et al.

(unpublished)

Ling et al. (1993) 


\begin{tabular}{|c|c|c|c|c|}
\hline Compound & Dose & $\begin{array}{l}\text { Host/parasite } \\
\text { stage }\end{array}$ & Efficacy & Reference \\
\hline & 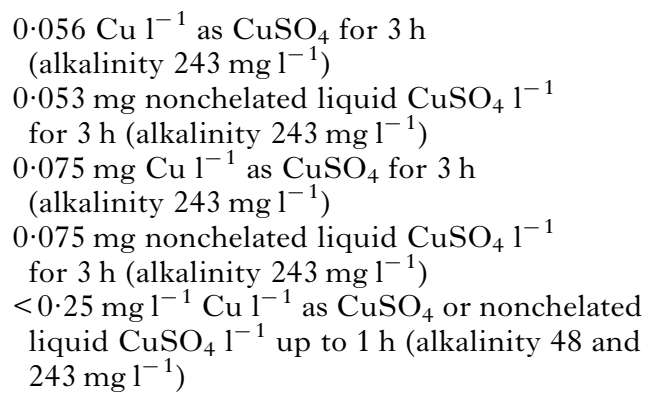 & & $\begin{array}{l}\text { Partially effective }-50 \% \text { mortality } \\
\text { Partially effective }-50 \% \text { mortality } \\
\text { Effective - 95\% mortality } \\
\text { Effective - } \sim 95 \% \text { mortality } \\
\text { Not effective - no reduction in theront survival }\end{array}$ & \\
\hline \multirow[t]{4}{*}{ In vivo - bath } & $\begin{array}{l}0 \cdot 05 \mathrm{mg} \mathrm{l}^{-1} \text { for } 10 \mathrm{~d} \\
0 \cdot 05 \mathrm{mg} \mathrm{l}^{-1} \text { daily for } 17 \mathrm{~d} \\
0 \cdot 1 \mathrm{mg} \mathrm{l}^{-1} \text { for } 10 \mathrm{~d} \\
0 \cdot 1 \mathrm{mg} \mathrm{l}^{-1} \text { for } 8 \mathrm{~d} \\
0 \cdot 1 \mathrm{mg} \mathrm{l}^{-1} \text { daily for } 17 \mathrm{~d} \\
0 \cdot 20 \mathrm{mg} \mathrm{l}^{-1} \text { daily for } 14 \mathrm{~d} \\
0 \cdot 25 \mathrm{mg} \mathrm{l}^{-1} \text { daily for } 14 \mathrm{~d} \\
255 \mu \mathrm{g} \mathrm{Cu}^{+2} 1^{-1} \text { for } 1 \text { week } \\
255 \mu \mathrm{g} \mathrm{Cu}^{+2} 1^{-1} \text { for } 2 \text { weeks } \\
255 \mu \mathrm{g} \mathrm{Cu}^{+2} \mathrm{l}^{-1} \text { for } 3 \text { weeks } \\
288 \mu \mathrm{g} \mathrm{Cu}^{+2} 1^{-1} \text { for } 15 \mathrm{~min} \\
288 \mu \mathrm{g} \mathrm{Cu}^{+2} 1^{-1} \text { for } 30 \mathrm{~min} \\
288 \mu \mathrm{g} \mathrm{Cu}^{+2} \mathrm{l}^{-1} \text { for } 60 \mathrm{~min} \\
288 \mu \mathrm{g} \mathrm{Cu}^{+2} \mathrm{l}^{-1} \text { for } 2 \mathrm{~h}\end{array}$ & $\begin{array}{l}\text { I. punctatus } \\
\text { B. bidyanus } \\
\text { I. punctatus } \\
\text { B. bidyanus } \\
\text { C. auratus }\end{array}$ & $\begin{array}{l}\text { Not effective }-100 \% \text { of infected fish died on day } 10 \\
\text { Not effective }- \text { treated fish remained infected } \\
\text { Effective }- \text { no trophonts found on treated fish } \\
\text { Not effective }- \text { all treated fish died on day } 13 \\
\text { Effective }- \text { treated fish free of trophonts } \\
\text { Effective }- \text { treated fish free of trophonts } \\
\text { Effective }- \text { treated fish free of trophonts } \\
\text { 'Effective' but } 11 \cdot 1 \% \text { of infected fish died } \\
\text { 'Effective' but } 33 \cdot 3 \% \text { of infected fish died } \\
\text { Not effective }-44 \cdot 4 \% \text { of infected fish died } \\
\text { Not effective }-100 \% \text { of infected fish died } \\
\text { Not effective }-66 \cdot 70 \% \text { of infected fish died } \\
\text { Not effective }-44 \cdot 4 \% \text { of infected fish died } \\
\text { 'Effective' but } 11 \cdot 1 \% \text { of infected fish died }\end{array}$ & $\begin{array}{l}\text { Schlenk et al. }(1998) \\
\text { Rowland } \text { et al. }(2009) \\
\text { Schlenk } \text { et al. }(1998) \\
\text { Rowland } \text { et al. }(2009)\end{array}$ \\
\hline & $\begin{array}{l}0.4 \mathrm{mg} \mathrm{l}^{-1} \text { for } 8 \mathrm{~d} \\
0.5 \mathrm{mg} \mathrm{l}^{-1} \text { for } 10 \mathrm{~d}\end{array}$ & I. punctatus & $\begin{array}{l}\text { Effective - no trophonts found on treated fish } \\
\text { Effective - no trophonts found on treated fish }\end{array}$ & Schlenk et al. (1998) \\
\hline & $\begin{array}{l}0 \cdot 5 \mathrm{mg} \mathrm{l}^{-1} \text { daily for } 17 \mathrm{~d} \\
0 \cdot 8 \mathrm{mg} \mathrm{l}^{-1} \text { for } 8 \mathrm{~d} \\
1 \mathrm{mg} \mathrm{l}^{-1} \text { for } 10 \mathrm{~d}\end{array}$ & $\begin{array}{l}\text { B. bidyanus } \\
\text { I. punctatus }\end{array}$ & $\begin{array}{l}\text { Not effective }-100 \% \text { of infected fish died } \dagger \\
\text { Effective - no trophonts found on treated fish } \\
\text { Effective - no trophonts found on treated fish }\end{array}$ & $\begin{array}{l}\text { Rowland } \text { et al. (2009) } \\
\text { Schlenk et al. (1998) }\end{array}$ \\
\hline & $1 \mathrm{mg} \mathrm{l}^{-1}$ daily for $17 \mathrm{~d}$ & B. bidyanus & Not effective $-100 \%$ of infected fish died $\dagger$ & Rowland et al. (2009) \\
\hline \multirow[t]{3}{*}{ (static tanks) } & $\begin{array}{l}1 \mathrm{mg}^{-1} \text { daily for } 11 \mathrm{~d} \text { (alkalinity } \\
68 \mathrm{mg}^{-1} \text { ) } \\
1 \mathrm{mg} \mathrm{l}^{-1} \text { daily for } 11 \mathrm{~d} \text { (alkalinity } 180 \\
\text { and } 250 \mathrm{mg} \mathrm{l}^{-1} \text { ) } \\
1 \mathrm{mg} \mathrm{l}^{-1} \text { alternate day for } 11 \mathrm{~d} \text { (alkalinity } \\
150 \mathrm{mg} \mathrm{l}^{-1} \text { ) }\end{array}$ & I. punctatus & $\begin{array}{l}\text { Not effective }-80-100 \% \text { of the treated infected fish } \\
\text { died } \\
\text { Not effective }-80 \% \text { of the treated infected fish died } \\
\text { Not effective }- \text { all the treated infected fish died }\end{array}$ & $\begin{array}{l}\text { Tieman and Goodwin } \\
(2001)\end{array}$ \\
\hline & $\begin{array}{l}1 \cdot 1 \mathrm{mg} \mathrm{l}^{-1}(8 \mathrm{~d} \text { trial }) \\
\text { Treatment: } \mathrm{d} 1,3,5 \text { and } 7\end{array}$ & I. punctatus & 'Effective' $-15 \%$ of the treated infected fish died & Straus (2008) \\
\hline & $\begin{array}{l}1.2 \mathrm{mg} \mathrm{l}^{-1} \text { for } 8 \mathrm{~d} \\
1.5 \mathrm{mg} \mathrm{l}^{-1} \text { for } 10 \mathrm{~d}\end{array}$ & $\begin{array}{l}\text { I. punctatus } \\
\text { I. punctatus }\end{array}$ & $\begin{array}{l}\text { Effective - no trophonts found on treated fish } \\
\text { Effective }- \text { no trophonts found on treated fish }\end{array}$ & Schlenk et al. (1998) \\
\hline
\end{tabular}

$<0.25 \mathrm{mg} \mathrm{l}^{-1} \mathrm{Cu} \mathrm{l}^{-1}$ as $\mathrm{CuSO}_{4}$ or nonchelated

liquid $\mathrm{CuSO}_{4} \mathrm{1}^{-1}$ up to $1 \mathrm{~h}$ (alkalinity 48 and $243 \mathrm{mg} \mathrm{l}^{-1}$ )

$0.05 \mathrm{mg} \mathrm{l}^{-1}$ daily for $17 \mathrm{~d}$

$0 \cdot 1 \mathrm{mg} \mathrm{l}^{-1}$ for $10 \mathrm{~d}$

$0 \cdot 1 \mathrm{mg} \mathrm{l}^{-1}$ for $8 \mathrm{~d}$

$0 \cdot 1 \mathrm{mg} \mathrm{l}^{-1}$ daily for $17 \mathrm{~d}$

$255 \mu \mathrm{g} \mathrm{Cu}^{+2} 1^{-1}$ for 3 weeks

for $2 \mathrm{~h}$

$4 \mathrm{mg} \mathrm{l}^{-1}$ for $8 \mathrm{c}$

$0.5 \mathrm{mg} \mathrm{l}^{-1}$ daily for $17 \mathrm{~d}$

1 for $8 \mathrm{~d}$

$\mathrm{mg} \mathrm{l}^{-1}$ daily for 17

I. punctat

B. bidyanus

I. punctatus

.

Effective - no trophonts found on treated fish

Not effective - all treated fish died on day 13

Effective' but $33.3 \%$ of infected fish died

phonts found on treated fish 
$1.5 \mathrm{mg} \mathrm{l}^{-1}$ daily for $11 \mathrm{~d}$ (alkalinity

$1.5 \mathrm{mg} \mathrm{l}^{-1}$ alternate day for $11 \mathrm{~d}$ (static tanks) $\left.68 \mathrm{mg} \mathrm{l}^{-1}\right)$

$1.5 \mathrm{mg} \mathrm{l}^{-1}$ daily for $11 \mathrm{~d}$ (alkalinity

180 and $250 \mathrm{mg} \mathrm{l}^{-1}$ )

(alkalinity $50 \mathrm{mg} \mathrm{l}^{-1}$ )

$1.6 \mathrm{mg} \mathrm{l}^{-1}$ for $8 \mathrm{~d}$

$2 \cdot 0 \mathrm{mg} \mathrm{l}^{-1}$ for $10 \mathrm{~d}$

$2 \cdot 2 \mathrm{mg} \mathrm{l}^{-1}(8 \mathrm{~d}$ trial $)$

Treatment: $\mathrm{d} 1,3,5$ and 7

$3 \cdot 3 \mathrm{mg} \mathrm{l}^{-1}(8 \mathrm{~d}$ trial $)$

Treatment: $\mathrm{d} 1,3,5$ and 7

$4 \cdot 4 \mathrm{mg} \mathrm{l}^{-1}(8 \mathrm{~d}$ trial $)$

Treatment: d1, 3, 5 and 7

Coyden

See entry clopidol (3, 5-dichloro-2, 6-dimethyl-4-pyridinol)

Decoquinate

In vivo - In-feed $100 \mathrm{mg} \mathrm{kg}^{-1}$ feed $10 \mathrm{~d}$ prior to infection

I. punctatus

I. punctatus

I. punctatus

I. punctatus

esirox

See entry for peracetic acid + acetic acid + hydrogen peroxide based formulations $\left(13 \% \mathrm{PAA}+20 \% \mathrm{AA}+20 \% \mathrm{H}_{2} \mathrm{O}_{2}\right)$

\section{Detarox}

See entry for peracetic acid + acetic acid + hydrogen peroxide based formulations

Diethylcarbamazine

In vivo-In-feed $40 \mathrm{~g} \mathrm{~kg}^{-1}$ feed for $10 \mathrm{~d} \quad$ O. mykiss

\section{Dimetridazole (DMZ)}

$\begin{array}{ll}\text { In vitro } & 5 \mathrm{mg} \mathrm{l}^{-1} \text { for } 3 \mathrm{~h} \\ & 5 \mathrm{mg} \mathrm{l}^{-1} \text { for } 24 \mathrm{~h}\end{array}$

In vivo-bath $\quad 28 \mathrm{mg} /$ feed mixed with lactate

$($ Emetryl $\mathbb{B})$ for $10 \mathrm{~d}$

In-feed

Theronts

Adults**

Tomocysts

O. mykiss

O. mykiss

Protomonts

In vitro

$100 \mathrm{mg} \mathrm{l}^{-1}$ for $2 \mathrm{~h}$

$200 \mathrm{mg} \mathrm{l}^{-1}$ for $2 \mathrm{~h}$

In vivo-bath treated fish
$5 \mathrm{mg} \mathrm{l}^{-1}$ for $24 \mathrm{~h}$

$40 \mathrm{~g} \mathrm{~kg}^{-1}$ feed for $10 \mathrm{~d}$
Not effective $-40 \%$ of the treated infected fish died

(treated fish remain infected)

Not effective - all the treated infected fish died

Not effective - all the treated infected fish died

Effective - no trophonts found on treated fish

Effective - no trophonts found on treated fish

Not effective $-81 \cdot 7 \%$ of the treated infected fish died Straus (2008)

Not effective $-98 \cdot 3 \%$ of the treated infected fish died

Not effective $-96 \cdot 7 \%$ of the treated infected fish died
Not effective - no reduction in trophont numbers on Shinn et al. (2003a)
Partially effective - lower trophont counts observed in skin scrapes $(60 \%$ of treated fish free of infection)

Not effective - no details

Not effective - no details

Not effective - no details

Effective - no visible signs of infection by day 7 p.i. Rapp (1995)

Not effective $-85 \%$ of treated fish have high numbers of trophonts

Partially effective $-75 \%$ mortality after $2 \mathrm{~h}$

Effective $-100 \%$ mortality after $45 \mathrm{~min}$

Not effective - all protomonts developed normally
Tojo-Rodriguez and Santamarina-Fernandez (2001)

Wahli et al. (1993)

Tojo-Rodriguez and Santamarina-Fernandez (2001)

Tojo-Rodriguez et al (1994)

Tojo-Rodriguez et al . (1994) 


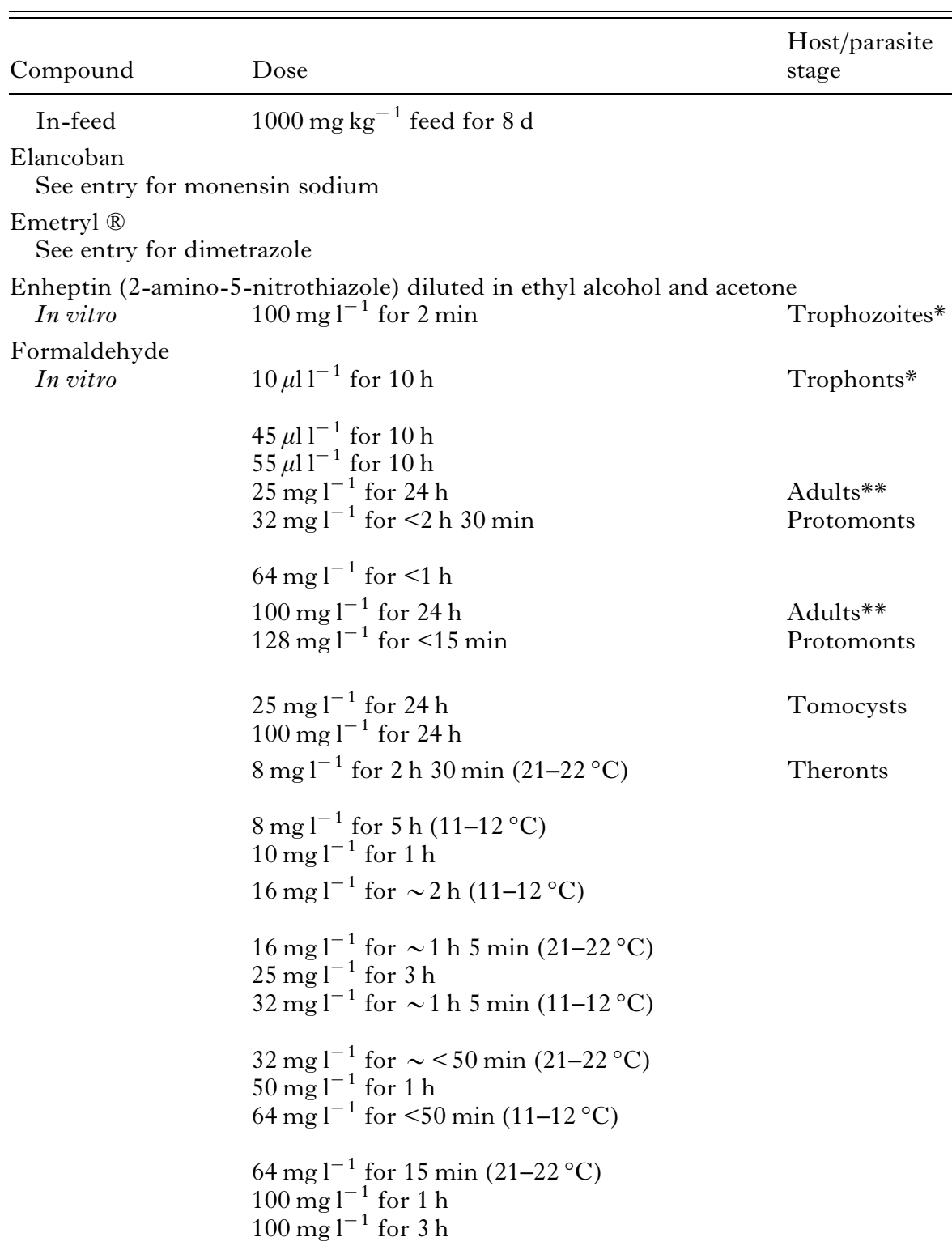

Efficacy

Reference

Not effective-all protomonts developed normally

ntry for dimetrazole
Effective $-100 \%$ mortality after $2 \mathrm{~h}$ post exposure

Not effective $-7 \%$ mortality

Effective $-7 \%$ mortality, no viable theronts produced

Effective $-100 \%$ mortality

Effective $-100 \%$ mortality after $24 \mathrm{~h}$

Effective $-100 \%$ mortality

Effective $-100 \%$ mortality

Effective $-100 \%$ mortality after $1 \mathrm{~h}$

Effective $-100 \%$ mortality

Effective $-100 \%$ mortality after $24 \mathrm{~h}$

Effective $-100 \%$ mortality after $24 \mathrm{~h}$

Effective $-100 \%$ mortality

Effective $-100 \%$ mortality

Not effective - 5\% mortality

Effective $-100 \%$ mortality

Effective $-100 \%$ mortality

Effective $-100 \%$ mortality after $30 \mathrm{~min}$

Effective $-100 \%$ mortality

Effective $-100 \%$ mortality

Effective $-100 \%$ mortality

Effective $-100 \%$ mortality

Not effective $-\sim 3 \%$ mortality

Effective $-100 \%$ mortality after $30 \mathrm{~min}$
Not effective $-\sim 10 \%$ mortality
Post and Vesley (1983)

Lahnsteiner and Weismann (2007)

Wahli et al. (1993)

Heinecke and Buchmann (2009)

Wahli et al. (1993)

Heinecke and Buchmann (2009)

Wahli et al. (1993)

Heinecke and Buchmann (2009)

Shinn et al. (2005)

Heinecke and Buchmann (2009)

Wahli et al. (1993)

Heinecke and Buchmann (2009)

Shinn et al. (2005)

Heinecke and Buchmann (2009)

Shinn et al. (2005) Wahli et al. (1993) 
$200 \mathrm{mg} \mathrm{l}^{-1}$ for $1 \mathrm{~h}$

In vivo-bath
$10 \mu \mathrm{ll}^{-1}$ for $6 \mathrm{~h}$ for $5 \mathrm{~d}$

$\left(18^{\circ} \mathrm{C}, 24 \mathrm{~h}\right.$ intervals $)$

$40 \mu \mathrm{l}^{-1}$ for $4 \mathrm{~h}$ for $5 \mathrm{~d}$

$\left(18^{\circ} \mathrm{C}, 24 \mathrm{~h}\right.$ intervals $)$

$80 \mu \mathrm{l}^{-1}$ for $1 \mathrm{~h}$ for $5 \mathrm{~d}$

$\left(18^{\circ} \mathrm{C}, 24 \mathrm{~h}\right.$ intervals $)$

$80 \mu \mathrm{ll}^{-1}$ for $2 \mathrm{~h}$ for $5 \mathrm{~d}$

$110 \mu \mathrm{ll}^{-1}$ for $2 \mathrm{~h}$ for $5 \mathrm{~d}$

$110 \mu \mathrm{ll}^{-1}$ for $1 \mathrm{~h}$ for $5 \mathrm{~d}$ $\left(18^{\circ} \mathrm{C}, 24 \mathrm{~h}\right.$ intervals $)$

$110 \mu \mathrm{ll}^{-1}$ for $1 \mathrm{~h}$ for $5 \mathrm{~d}$

$\left(10^{\circ} \mathrm{C}, 48 \mathrm{~h}\right.$ intervals $)$

$110 \mu \mathrm{l}^{-1}$ for $1 \mathrm{~h}$ for $5 \mathrm{~d}$

$\left(18^{\circ} \mathrm{C}, 24 \mathrm{~h}\right.$ intervals)

$110 \mu \mathrm{ll}^{-1}$ for $1 \mathrm{~h}$ for $5 \mathrm{~d}$

$\left(18^{\circ} \mathrm{C}, 48 \mathrm{~h}\right.$ intervals $)$

$110 \mu \mathrm{l}^{-1}$ for $1 \mathrm{~h}$ for $5 \mathrm{~d}$

$\left(25^{\circ} \mathrm{C}, 24 \mathrm{~h}\right.$ intervals $)$

$110 \mu 11^{-1}$ for $12 \mathrm{~h}\left(25^{\circ} \mathrm{C}\right)$

$110 \mu \mathrm{l}^{-1}$ for $3 \times 1 \mathrm{~h}$ for $5 \mathrm{~d}$

$\left(25^{\circ} \mathrm{C}, 24 \mathrm{~h}\right.$ intervals $)$

$110 \mu 11^{-1}$ for $5 \times 1 \mathrm{~h}$ for $5 \mathrm{~d}$

$\left(25^{\circ} \mathrm{C}, 24 \mathrm{~h}\right.$ intervals $)$

$110 \mu \mathrm{ll}^{-1}$ for $7 \times 1 \mathrm{~h}$ for $5 \mathrm{~d}$

$\left(25^{\circ} \mathrm{C}, 24 \mathrm{~h}\right.$ intervals $)$

$110 \mu \mathrm{l}^{-1}$ for $12 \mathrm{~h}\left(18^{\circ} \mathrm{C}\right)$

$110 \mu \mathrm{ll}^{-1}$ for $3 \times 1 \mathrm{~h}$ for $5 \mathrm{~d}$

$\left(18^{\circ} \mathrm{C}, 24 \mathrm{~h}\right.$ intervals $)$

$110 \mu \mathrm{l}^{-1}$ for $5 \times 1 \mathrm{~h}$ for $5 \mathrm{~d}$

$\left(18^{\circ} \mathrm{C}, 24 \mathrm{~h}\right.$ intervals)

$110 \mu \mathrm{ll}^{-1}$ for $7 \times 1 \mathrm{~h}$ for $5 \mathrm{~d}$

$\left(18^{\circ} \mathrm{C}, 24 \mathrm{~h}\right.$ intervals $)$

$0 \cdot 1,0 \cdot 15$ and $0 \cdot 2 \mathrm{ml}^{-1}$ for $1 \mathrm{~h}$
O. mykiss

Not effective - 40\% mortality

Shinn et al. (2005)

Not effective $-0 \%$ survival of treated fish on $\mathrm{d} 1$ and 3

Not effective $-30 \%$ survival of treated fish on $\mathrm{d} 1 ; 0 \%$ survival on $\mathrm{d} 3$

Effective $-50 \%$ survival on treated fish on $\mathrm{d} 1$ and 3 ; no trophonts seen on fish on $\mathrm{d} 1$ and 3

Effective - all treated fish survived; low number of trophonts on $\mathrm{d} 1$ and 3

ffective - all treated fish survived; no trophonts on d1 and 3

O. mykiss

Effective $-90 \%$ survival of treated fish on $\mathrm{d} 1$ and $3 ; \mathrm{no}$ trophonts on $\mathrm{d} 1$ and 3

Effective $-100 \%$ survival of treated fish on $\mathrm{d} 1$ and 3 ; Lahnsteiner and no trophonts on $\mathrm{d} 1$ and 3

Effective $-100 \%$ survival of treated fish on $\mathrm{d} 1$ and 3 ; no trophonts on $\mathrm{d} 1$ and 3

Not effective $-50 \%$ and $10 \%$ survival of treated fish on $\mathrm{d} 1$ and 3 ; heavy infections on $\mathrm{d} 1$ and 3

Not effective $-0 \%$ survival of treated fish on $\mathrm{d} 1$ and 3 heavy infections on $\mathrm{d} 1$ and 3

O. mykiss

effective $-100 \%$ and $10 \%$ survival of treated fish on $\mathrm{d} 1$ and 3 ; moderate and heavy infections on $\mathrm{d} 1$ and 3

Not effective $-70 \%$ and $0 \%$ survival of treated fish on $\mathrm{d} 1$ and 3 ; Moderate infection on d1

Not effective $-30 \%$ and $0 \%$ survival of treated fish on $\mathrm{d} 1$ and 3 ; moderate infection on $\mathrm{d} 1$

Not effective $-0 \%$ and $0 \%$ survival of treated fish on $\mathrm{d} 1$ and 3 ; heavy infection on $\mathrm{d} 1$

Not effective $-100 \%$ and $10 \%$ survival of treated fish on $\mathrm{d} 1$ and 3 medium and very heavy infections on $\mathrm{d} 1$ and 3

Not effective $-100 \%$ and $40 \%$ survival of treated fish on $\mathrm{d} 1$ and 3; low and heavy infections on $\mathrm{d} 1$ and 3 Effective $-100 \%$ survival of treated fish on $\mathrm{d} 1$ and 3 ; no infection on $\mathrm{d} 1$ and 3

Effective $-100 \%$ survival of treated fish on $\mathrm{d} 1$ and 3 ; no infection on $\mathrm{d} 1$ and 3

O. mykiss

Effective - reduction in the number of trophonts on treated fish

S. fontinalis 


\begin{tabular}{|c|c|c|c|c|}
\hline Compound & Dose & $\begin{array}{l}\text { Host/parasite } \\
\text { stage }\end{array}$ & Efficacy & Reference \\
\hline & $\begin{array}{l}10 \mathrm{mg} \mathrm{l}^{-1} \text { for } 17 \mathrm{~d} \\
20 \mathrm{mg} \mathrm{l}^{-1} \text { for } 17 \mathrm{~d}\end{array}$ & B. bidyanus & $\begin{array}{l}\text { Not effective }- \text { all treated infected fish died } \\
\text { Not effective - all treated fish still infected }\end{array}$ & Rowland et al. (2009) \\
\hline & $25 \mathrm{mg} \mathrm{l}^{-1}-2 \times$ for $1 \mathrm{~h}$ & O. mykiss & $\begin{array}{l}\text { Not effective - not specified but fish survival } \\
\text { compromised }\end{array}$ & Wahli et al. (1993) \\
\hline & $\begin{array}{l}\text { 1st: day } 9 \mathrm{p} . \mathrm{i}, 2 \mathrm{nd} \text { : day } 12 \mathrm{p.i} \\
25 \mathrm{mg} \mathrm{l}^{-1} \text { for } 4 \text { h for } 4 \mathrm{~d} \text { week }\end{array}$ & I. punctatus & Not effective $-40-70 \%$ of treated infected fish died & Bodensteiner et al. (2000) \\
\hline \multirow[t]{11}{*}{ (static tanks) } & $25 \mathrm{mg}^{-1}$ alternate days for $20 \mathrm{~d}$ & I. punctatus & Not effective - all treated infected fish died & $\begin{array}{l}\text { Tieman and Goodwin } \\
(2001)\end{array}$ \\
\hline & $25 \mathrm{mg} \mathrm{l}^{-1}$ daily for $20 \mathrm{~d}$ & & $\begin{array}{l}\text { Partially effective }-20-60 \% \text { of treated infected fish } \\
\text { died }\end{array}$ & \\
\hline & $25 \mathrm{mg} \mathrm{l}^{-1} 3-4$ times a week for 6 weeks & S. salar & Not effective - parasite load increased on treated fish & $\begin{array}{l}\text { Rintamäki-Kinnunen } \\
\text { et al. }(2005 b)\end{array}$ \\
\hline & $25 \mathrm{mg} \mathrm{l}^{-1} 3-4$ times a week for 6 weeks & S. trutta & Not effective-parasite load increased on treated fish & $\begin{array}{l}\text { Rintamäki-Kinnunen } \\
\text { et al. }(2005 b)\end{array}$ \\
\hline & $30 \mathrm{mg} \mathrm{l}^{-1}$ for $17 \mathrm{~d}$ & B. bidyanus & Effective - no trophonts found on treated fish & Rowland et al. (2009) \\
\hline & $50 \mathrm{mg} \mathrm{l}^{-1}$ alternate day for $20 \mathrm{~d}$ & I. punctatus & Not effective - all treated infected fish died & $\begin{array}{l}\text { Tieman and Goodwin } \\
(2001)\end{array}$ \\
\hline & $50 \mathrm{mg} \mathrm{l}^{-1}$ daily for $20 \mathrm{~d}$ & & $\begin{array}{l}\text { Partially effective }-20-60 \% \text { of treated infected fish } \\
\text { died }\end{array}$ & \\
\hline & $50 \mathrm{mg} \mathrm{l}^{-1} 3-4$ times a week for 6 weeks & S. salar & Not effective - parasite load increased on treated fish & $\begin{array}{l}\text { Rintamäki-Kinnunen } \\
\text { et al. }(2005 b)\end{array}$ \\
\hline & $50 \mathrm{mg} \mathrm{l}^{-1} 3-4$ times a week & S. trutta & Not effective - parasite load increased on treated fish & \\
\hline & $100 \mathrm{mg} \mathrm{l}^{-1}-2 \times$ for $1 \mathrm{~h}$ & O. mykiss & $\begin{array}{l}\text { Not effective - not specified but fish survival } \\
\text { compromised }\end{array}$ & Wahli et al. (1993) \\
\hline & 1st: day 9 p.i, 2nd: day 12 p.i & & & \\
\hline \multirow{2}{*}{$\begin{array}{l}\text { (static tanks) } \\
\text { (flow through) }\end{array}$} & $100 \mathrm{mg} \mathrm{l}^{-1}$ daily for $20 \mathrm{~d}$ & I. punctatus & Not effective - all treated infected fish died & $\begin{array}{l}\text { Tieman and Goodwin } \\
(2001)\end{array}$ \\
\hline & $\begin{array}{l}100 \mathrm{mg} \mathrm{l}^{-1} \text { alternate day for } 20 \mathrm{~d} \\
60-250 \mathrm{mg} \mathrm{l^{-1 }} \text { for } 20 \mathrm{~min}-1 \mathrm{~h}\end{array}$ & Not specified & $\begin{array}{l}\text { Not effective - all treated infected fish died } \uparrow \\
\text { 'Effective' - efficacy though not specified }\end{array}$ & $\begin{array}{l}\text { Rahkonen and Koski } \\
(2002)\end{array}$ \\
\hline \multicolumn{5}{|c|}{ Formaldehyde + Desirox $(13 \%$ peracetic acid, $20 \%$ acetic acid and $20 \%$ hydrogen peroxide) } \\
\hline \multirow[t]{4}{*}{ In vivo-bath } & $\begin{array}{l}25+10 \mathrm{mg} \mathrm{l}^{-1} 3-4 \text { times a for } \\
4 \text { weeks week }\end{array}$ & S. salar & & $\begin{array}{l}\text { Rintamäki-Kinnunen } \\
\text { et al. }(2005 b)\end{array}$ \\
\hline & $\begin{array}{l}50+10 \mathrm{mg} \mathrm{l}^{-1} 3-4 \text { times a week } \\
\text { for } 4 \text { weeks }\end{array}$ & & 'Effective' - parasite load reduced on treated fish & \\
\hline & $\begin{array}{l}100+10 \mathrm{mg} \mathrm{l}^{-1} 3 \text { times a week } \\
\text { for } 4 \text { weeks }\end{array}$ & S. salar & Trial inconclusive-details missing & $\begin{array}{l}\text { Rintamäki-Kinnunen } \\
\text { et al. }(2005 a)\end{array}$ \\
\hline & $\begin{array}{l}123+8 \mathrm{mg} \mathrm{l}^{-1} 4 \text { times a week } \\
\text { for } 5 \text { weeks }\end{array}$ & & Not effective - parasite load increased on treated fish & $\begin{array}{l}\text { Rintamäki-Kinnunen } \\
\text { et al. }(2005 a)\end{array}$ \\
\hline
\end{tabular}


Rintamäki-Kinnunen et al. $(2005 a)$

Formaldehyde + malachite green

$100+0 \cdot 4 \mathrm{mg} \mathrm{l}^{-1}$ for $24 \mathrm{~h}$

$25+0 \cdot 1 \mathrm{mg} \mathrm{l}^{-1}$ for $24 \mathrm{~h}$

$100+0 \cdot 4 \mathrm{mg} \mathrm{l}^{-1}$ for $24 \mathrm{~h}$

$25+0 \cdot 1 \mathrm{mg} \mathrm{l}^{-1}$ for $3 \mathrm{~h}$

$100+0 \cdot 4 \mathrm{mg} \mathrm{l}^{-1}$ for $3 \mathrm{~h}$

In vivo-bath

$25+0 \cdot 05 \mathrm{mg}^{-1}$

$25+0 \cdot 1 \mathrm{mg} \mathrm{l}^{-1}-2 \times$ for $1 \mathrm{~h}$

Adults**

Effective $-100 \%$ mortality after $24 \mathrm{~h}$

Effective $-100 \%$ mortality after $24 \mathrm{~h}$

Tomocysts

Theronts

Effective $-100 \%$ mortality after $24 \mathrm{~h}$

Effective $-100 \%$ mortality after $24 \mathrm{~h}$

Effective $-100 \%$ mortality after $30 \mathrm{~min}$

Effective $-100 \%$ mortality after 5 min

Cichla ocellaris

O. mykiss

'Effective' - efficacy not specified

Partially effective against the parasites - efficacy not specified

Partially effective against the parasites - efficacy not specified

Partially effective - small increases in parasite

burdens observed over infection period

Trial inconclusive - low parasite numbers across all

groups

Trophozoites*

Effective $-30 \%$ mortality after $12 \mathrm{~h}, 80 \%$ after $24 \mathrm{~h}$ post exposure

Not effective - no details

Effective $-100 \%$ mortality after $24 \mathrm{~h}$

Tomocysts

Theronts

effective - no details

$00 \mathrm{mg} \mathrm{l}^{-1}$ for 3

O. mykiss

$25 \mathrm{mg} \mathrm{l}^{-1}-2 \times$ for $1 \mathrm{~h}$

1 st: day 9 p.i, 2 nd: day $12 \mathrm{p}$.

In-feed

$50 \mathrm{mg} \mathrm{kg}^{-1}$ fish for $10 \mathrm{~d}$

Not effective - no details

Not effective - no details

Furoxone (3-[(5-nitrofuran-2-yl) methylideneamino]-1,3-oxazolidin-2-one) diluted in ethyl alcohol and acetone
In vitro
$100 \mathrm{mg} \mathrm{l}^{-1}$ for $2 \mathrm{~min}$
Trophozoites*

Not effective $-0 \%$ mortality after $24 \mathrm{~h}$ post exposure

$\beta$-Glucan (from Saccharomyces cerevisiae)

In vivo - In-feed $\quad 0 \cdot 2 \%$ for $14 \mathrm{~d}$ prior inf.

$0 \cdot 2 \%$ for $35 \mathrm{~d}$ prior inf.

$\mathrm{Hb} \beta \mathrm{P}-1$ (peptide from the $\beta$-haemoglobin peptide family)

In vitro
Wahli et al. (1993)

Guest (1983)

Wahli et al. (1993)

Rintamäki-Kinnunen et al. $(2005 a)$

Post and Vesley (1983)

Wahli et al. (1993)

Wahli et al. (1993)

Post and Vesley (1983)

Lauridsen and

Buchmann (2010)

Ullal and Noga (2010)
O. mykiss

Trophonts* $(323 \mu \mathrm{m})$

$(222 \mu \mathrm{m})$

$(500 \mu \mathrm{m})$

$(323 \mu \mathrm{m})$

$(231 \mu \mathrm{m})$

$(519 \mu \mathrm{m})$
Not effective $-14 \%$ trophont reduction

Not effective $-18 \%$ trophont reduction

Effective $-100 \%$ mortality

Effective $-100 \%$ mortality

Effective $-100 \%$ mortalit

Effective $-100 \%$ mortalit

Effective $-100 \%$ mortality

Effective $-100 \%$ mortality

$25 \mu \mathrm{g} \mathrm{ml}^{-1}$ for $1 \mathrm{~min} 54$
$25 \mu \mathrm{g} \mathrm{ml}^{-1}$ for $4 \mathrm{~min} 14$ 


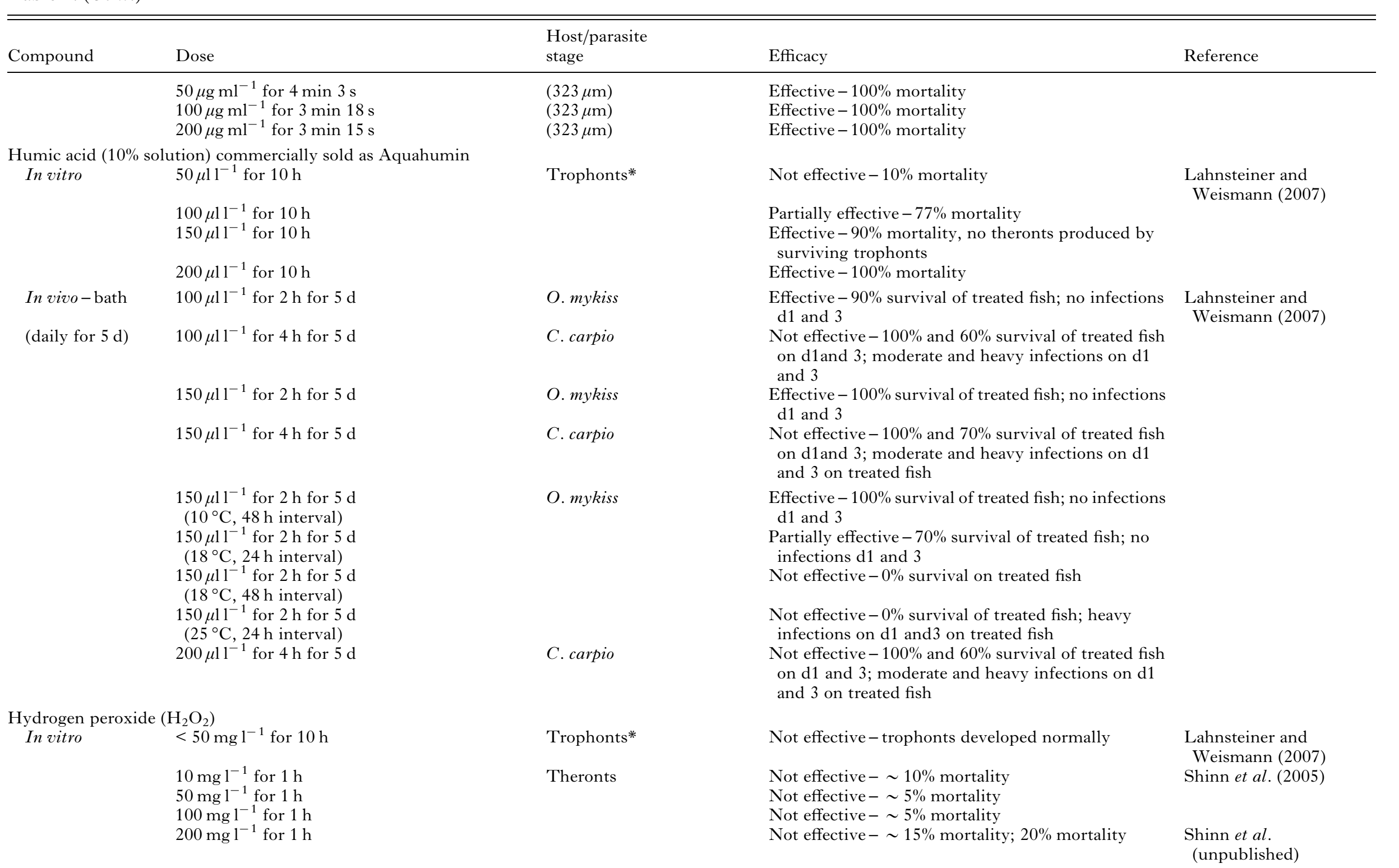


Hydrogen peroxide + acetic acid based formulation commercialized as Perotan

In vitro $50 \mu 1^{-1}$ for $10 \mathrm{~h}$ Trophonts*

$100 \mu \mathrm{ll}^{-1}$ for $10 \mathrm{~h}$

Not effective $-7 \%$ mortality

Lahnsteiner and

Weismann (2007)

Incimaxx Aquatic

See entry for peracetic acid + acetic acid + hydrogen peroxide + peroctanoic acid based formulation Iodine

$\begin{array}{lll}\text { In vivo-bath } & 0.25 \mathrm{mg} \mathrm{l}^{-1} \text { daily for } 11 \mathrm{~d} & \text { I. punctatus } \\ \text { (static tanks) } & 0.50 \mathrm{mg} \mathrm{l}^{-1} \text { daily for } 11 \mathrm{~d} \\ & 1.00 \mathrm{mg} \mathrm{l}^{-1} \text { daily for } 11 \mathrm{~d} & \\ 1.00 \mathrm{mg} \mathrm{l}^{-1} \text { for } 11 \text { d (alternate days) }\end{array}$

$1 \cdot 00 \mathrm{mg} \mathrm{l}^{-1}$ for $11 \mathrm{~d}$ (alternate days)

Ivermectin commercialised as Ivomec

In vitro $\quad<50 \mathrm{mg} \mathrm{l}^{-1}$ for $10 \mathrm{~h}$

Trophonts*

Effective $-100 \%$ mortality

Not effective - all treated infected fish died

Not effective - all treated infected fish died

Not effective - all treated infected fish died

Not effective - all treated infected fish died

Not effective - trophonts developed normally

Tieman and Goodwin (2001)

Ivomec

See entry for Ivermectin

Ketoconazole

In vitro

$200 \mathrm{mg} \mathrm{l}^{-1}$ for $2 \mathrm{~h}$

In vivo-bath

$200 \mathrm{mg} \mathrm{l}^{-1}$ for $3 \mathrm{~h}$

O. mykiss

In-feed

$1000 \mathrm{mg} \mathrm{kg}^{-1}$ feed for $8 \mathrm{~d}$

$40 \mathrm{~g} \mathrm{~kg}^{-1}$ feed for $10 \mathrm{~d}$

O. mykiss

Levamisole

In vivo-In-feed $40 \mathrm{~g} \mathrm{~kg}^{-1}$ feed for $10 \mathrm{~d} \quad$ O. mykiss

Macleaya cordata (active compound sanguinarine)

$\begin{array}{lll}\text { In vitro } & 60 \mathrm{mg} \mathrm{l}^{-1} \text { for } 4 \mathrm{~h} & \text { Trophonts* } \\ \text { (Butanol extract) } & 80 \mathrm{mg} \mathrm{l}^{-1} \text { for } 4 \mathrm{~h} & \end{array}$

(Butanol extract) $80 \mathrm{mg} \mathrm{l}^{-1}$ for $4 \mathrm{~h}$

$100 \mathrm{mg} \mathrm{l}^{-1}$ for $4 \mathrm{~h}$

$120 \mathrm{mg} \mathrm{l}^{-1}$ for $4 \mathrm{~h}$

(Chloroform extract)

$30 \mathrm{mg} \mathrm{l}^{-1}$ for $4 \mathrm{~h}$
$50 \mathrm{mg}^{-1}$ for $4 \mathrm{~h}$

Effective $-0 \%$ mortality after $2 \mathrm{~h}$ but protomonts do not develop

Not effective - all trophonts develop normally

ojo-Rodriguez et al

(1994)

Tojo-Rodriguez et al

Not effective - toxic to the fish; all trophonts develop

normally

Partially effective $-50 \%$ of medicated fish with low numbers of trophonts

Tojo-Rodriguez and

Santamarina-Fernandez (2001)

Not effective $-40 \%$ of medicated fish with low numbers of trophonts

Tojo-Rodriguez and

Santamarina-Fernandez (2001)

Not effective $-32 \cdot 5 \%$ mortality

Not effective $-47 \cdot 5 \%$ mortality

Partially effective $-65 \%$ mortality

Partially effective $-70 \%$ mortality

Partially effective $-67 \cdot 5 \%$ mortality

Effective $-82 \cdot 5 \%$ mortality 


\begin{tabular}{|c|c|c|c|c|}
\hline Compound & Dose & $\begin{array}{l}\text { Host/parasite } \\
\text { stage }\end{array}$ & Efficacy & Reference \\
\hline & $\begin{array}{l}70 \mathrm{mg} \mathrm{l}^{-1} \text { for } 4 \mathrm{~h} \\
90 \mathrm{mg} \mathrm{l}^{-1} \text { for } 4 \mathrm{~h}\end{array}$ & & $\begin{array}{l}\text { Effective }-100 \% \text { mortality } \\
\text { Effective }-100 \% \text { mortality }\end{array}$ & \\
\hline (Ethyl acetate & $\begin{array}{l}60 \mathrm{mg} \mathrm{l}^{-1} \text { for } 4 \mathrm{~h} \\
80 \mathrm{mg} \mathrm{l}^{-1} \text { for } 4 \mathrm{~h} \\
100 \mathrm{mg} \mathrm{l}^{-1} \text { for } 4 \mathrm{~h} \\
120 \mathrm{mg} \mathrm{l}^{-1} \text { for } 4 \mathrm{~h}\end{array}$ & & $\begin{array}{l}\text { Not effective }-37 \cdot 5 \% \text { mortality } \\
\text { Partially effective }-52 \cdot 5 \% \text { mortality } \\
\text { Effective }-80 \% \text { mortality } \\
\text { Effective }-80 \% \text { mortality }\end{array}$ & \\
\hline (Petroleum et & $\begin{array}{l}60 \mathrm{mg} \mathrm{l}^{-1} \text { for } 4 \mathrm{~h} \\
80 \mathrm{mg} \mathrm{l}^{-1} \text { for } 4 \mathrm{~h} \\
100 \mathrm{mg} \mathrm{l}^{-1} \text { for } 4 \mathrm{~h} \\
120 \mathrm{mg} \mathrm{l}^{-1} \text { for } 4 \mathrm{~h}\end{array}$ & & $\begin{array}{l}\text { Not effective }-27 \cdot 5 \% \text { mortality } \\
\text { Not effective }-30 \% \text { mortality } \\
\text { Not effective }-30 \% \text { mortality } \\
\text { Not effective }-40 \% \text { mortality }\end{array}$ & \\
\hline (Water extract & $\begin{array}{l}60 \mathrm{mg} \mathrm{l}^{-1} \text { for } 4 \mathrm{~h} \\
80 \mathrm{mg} \mathrm{l}^{-1} \text { for } 4 \mathrm{~h} \\
100 \mathrm{mg} \mathrm{l}^{-1} \text { for } 4 \mathrm{~h} \\
120 \mathrm{mg} \mathrm{l}^{-1} \text { for } 4 \mathrm{~h}\end{array}$ & & $\begin{array}{l}\text { Not effective }-7 \cdot 5 \% \text { mortality } \\
\text { Not effective }-17 \cdot 5 \% \text { mortality } \\
\text { Not effective }-25 \% \text { mortality } \\
\text { Not effective }-27 \cdot 5 \% \text { mortality }\end{array}$ & \\
\hline $\begin{array}{l}\text { (Fractions fro } \\
\text { (Fraction } \mathrm{A})\end{array}$ & $\begin{array}{l}\text { hloroform extract) } \\
10 \mathrm{mg} \mathrm{l}^{-1} \text { for } 4 \mathrm{~h} \\
20 \mathrm{mg} \mathrm{l}^{-1} \text { for } 4 \mathrm{~h} \\
30 \mathrm{mg} \mathrm{l}^{-1} \text { for } 4 \mathrm{~h}\end{array}$ & Trophonts* & $\begin{array}{l}\text { Not effective }-12 \cdot 5 \% \text { mortality } \\
\text { Not effective }-15 \% \text { mortality } \\
\text { Not effective }-22 \cdot 5 \% \text { mortality }\end{array}$ & Yao et al. (2010) \\
\hline (Fraction B) & $\begin{array}{l}10 \mathrm{mg} \mathrm{l}^{-1} \text { for } 4 \mathrm{~h} \\
20 \mathrm{mg} \mathrm{l}^{-1} \text { for } 4 \mathrm{~h} \\
30 \mathrm{mg} \mathrm{l}^{-1} \text { for } 4 \mathrm{~h}\end{array}$ & & $\begin{array}{l}\text { Not effective }-12 \cdot 5 \% \text { mortality } \\
\text { Not effective }-20 \% \text { mortality } \\
\text { Not effective }-25 \% \text { mortality }\end{array}$ & \\
\hline (Fraction $\mathrm{C})$ & $\begin{array}{l}10 \mathrm{mg} \mathrm{l}^{-1} \text { for } 4 \mathrm{~h} \\
20 \mathrm{mg} \mathrm{l}^{-1} \text { for } 4 \mathrm{~h} \\
30 \mathrm{mg} \mathrm{l}^{-1} \text { for } 4 \mathrm{~h}\end{array}$ & & $\begin{array}{l}\text { Not effective }-42 \cdot 5 \% \text { mortality } \\
\text { Not effective }-67 \cdot 5 \% \text { mortality } \\
\text { Not effective }-87 \cdot 5 \% \text { mortality }\end{array}$ & \\
\hline (Fraction D) & $\begin{array}{l}5 \mathrm{mg} \mathrm{l}^{-1} \text { for } 4 \mathrm{~h} \\
9 \mathrm{mg} \mathrm{l}^{-1} \text { for } 4 \mathrm{~h} \\
11 \mathrm{mg} \mathrm{l}^{-1} \text { for } 4 \mathrm{~h}\end{array}$ & & $\begin{array}{l}\text { Effective }-90 \% \text { mortality } \\
\text { Effective }-100 \% \text { mortality } \\
\text { Effective }-100 \% \text { mortality }\end{array}$ & \\
\hline (Fraction E) & $\begin{array}{l}10 \mathrm{mg} \mathrm{l}^{-1} \text { for } 4 \mathrm{~h} \\
20 \mathrm{mg} \mathrm{l}^{-1} \text { for } 4 \mathrm{~h} \\
30 \mathrm{mg} \mathrm{l}^{-1} \text { for } 4 \mathrm{~h}\end{array}$ & & $\begin{array}{l}\text { Partially effective }-55 \% \text { mortality } \\
\text { Partially effective }-77 \cdot 5 \% \text { mortality } \\
\text { Effective }-92 \cdot 5 \% \text { mortality }\end{array}$ & \\
\hline (Fraction G) & $\begin{array}{l}10 \mathrm{mg} \mathrm{l}^{-1} \text { for } 4 \mathrm{~h} \\
20 \mathrm{mg} \mathrm{l}^{-1} \text { for } 4 \mathrm{~h} \\
30 \mathrm{mg} \mathrm{l}^{-1} \text { for } 4 \mathrm{~h} \\
10 \mathrm{mg} \mathrm{l}^{-1} \text { for } 4 \mathrm{~h} \\
20 \mathrm{mg} \mathrm{l}^{-1} \text { for } 4 \mathrm{~h} \\
30 \mathrm{mg} \mathrm{l}^{-1} \text { for } 4 \mathrm{~h}\end{array}$ & & $\begin{array}{l}\text { Not effective }-7 \cdot 5 \% \text { mortality } \\
\text { Not effective }-20 \% \text { mortality } \\
\text { Not effective }-25 \% \text { mortality } \\
\text { Not effective }-5 \% \text { mortality } \\
\text { Not effective }-7 \cdot 5 \% \text { mortality } \\
\text { Not effective }-15 \% \text { mortality }\end{array}$ & \\
\hline
\end{tabular}


(Compounds from Fraction D)

(Compound I) $\quad 5 \mathrm{mg} \mathrm{l}^{-1}$ for $4 \mathrm{~h}$

$7 \mathrm{mg} \mathrm{l}^{-1}$ for $4 \mathrm{~h}$

$9 \mathrm{mg} \mathrm{l}^{-1}$ for $4 \mathrm{~h}$

(Compound II) $5 \mathrm{mgl}^{-1}$ for $4 \mathrm{~h}$

$7 \mathrm{mgl}^{-1}$ for $4 \mathrm{~h}$

$9 \mathrm{mg} \mathrm{l}^{-1}$ for $4 \mathrm{~h}$

(Compound III) $\quad 0.5 \mathrm{mg} \mathrm{l}^{-1}$ for $4 \mathrm{~h}$ $0.9 \mathrm{mg} \mathrm{l}^{-1}$ for $4 \mathrm{~h}$ $1 \cdot 3 \mathrm{mg} \mathrm{l}^{-1}$ for $4 \mathrm{~h}$

(Compound IV) $5 \mathrm{mgl}^{-1}$ for $4 \mathrm{~h}$ $7 \mathrm{mg} \mathrm{l}^{-1}$ for $4 \mathrm{~h}$ $9 \mathrm{mg} \mathrm{l}^{-1}$ for $4 \mathrm{~h}$

In vivo-bath

(Compound I)

$0 \cdot 2 \mathrm{mg} \mathrm{l}^{-1}$ for $48 \mathrm{~h}$

$0 \cdot 3 \mathrm{mg} \mathrm{l}^{-1}$ for $48 \mathrm{~h}$

$0 \cdot 4 \mathrm{mg} \mathrm{l}^{-1}$ for $48 \mathrm{~h}$

$0 \cdot 5 \mathrm{mg} \mathrm{l}^{-1}$ for $48 \mathrm{~h}$

$0 \cdot 6 \mathrm{mg} \mathrm{l}^{-1}$ for $48 \mathrm{~h}$

$0 \cdot 7 \mathrm{mg} \mathrm{l}^{-1}$ for $48 \mathrm{~h}$

$0.8 \mathrm{mg} \mathrm{l}^{-1}$ for $48 \mathrm{~h}$

$0.9 \mathrm{mg} \mathrm{l}^{-1}$ for $48 \mathrm{~h}$

Malachite green

In vitro

$1 \mathrm{mg} \mathrm{l}^{-1}$ for $24 \mathrm{~h}$

$0 \cdot 15 \mathrm{mg} \mathrm{l}^{-1}$ for $24 \mathrm{~h}$

$1 \mathrm{mg} \mathrm{l}^{-1}$ for $24 \mathrm{~h}$

$0.004 \mathrm{mg} \mathrm{l}^{-1}$ for $1 \cdot 5,3$ and $15 \mathrm{~h}$

$0.02 \mathrm{mg} \mathrm{l}^{-1}$ for $1 \cdot 5,3$ and $15 \mathrm{~h}$

$0 \cdot 10 \mathrm{mg} \mathrm{l}^{-1}$ for 1.5 and $3 \mathrm{~h}$

$0 \cdot 10 \mathrm{mg} \mathrm{l}^{-1}$ for $15 \mathrm{~h}$

$1 \mathrm{mg} \mathrm{l}^{-1}$ for $3 \mathrm{~h}$

$50 \mathrm{mg} \mathrm{l}^{-1}$ for $15 \mathrm{~h}$

$50 \mathrm{mg} \mathrm{l}^{-1}$ for $1 \cdot 5,3$ and $15 \mathrm{~h}$

In vivo - bath

$25 \mathrm{mg} \mathrm{l}^{-1}-2 \times$ for $1 \mathrm{~h}$

1 st: day 9 p.i, 2 nd: day 12 p.i

$0 \cdot 1 \mathrm{mg} \mathrm{l}^{-1}$ daily for $20 \mathrm{~d}$ (static tanks)
Trophonts*

Not effective $-17 \cdot 5 \%$ mortality

Not effective $-22.5 \%$ mortality

Not effective $-22 \cdot 5 \%$ mortalit

Not effective $-22 \cdot 5 \%$ mortality

Not effective $-30 \%$ mortality

Not effective $-37 \cdot 5 \%$ mortality

Effective $-87 \cdot 5 \%$ mortality

Effective $-100 \%$ mortality

Effective $-100 \%$ mortality

Not effective $-5 \%$ mortality

Not effective $-7 \cdot 5 \%$ mortality

Not effective $-22 \cdot 5 \%$ mortality

Ctenopharyngodo idella

Tot effective $-16 \cdot 1 \%$ trophont reduction on treated fish

Not effective $-17 \cdot 3 \%$ trophont reduction on treated insh

Not effective $-32.9 \%$ trophont reduction on treated fish

Partially effective $-53 \cdot 9 \%$ trophont reduction on treated fish

Partially effective $-75 \cdot 3 \%$ trophont reduction on

treated fish

Effective $-82 \cdot 3 \%$ trophont reduction on treated fish

Effective $-89 \cdot 4 \%$ trophont reduction on treated fish

Effective $-96.8 \%$ trophont reduction on treated fish

Adults**

Tomocysts

Tomocysts

Theronts

O. mykiss

I. punctatus
Effective $-100 \%$ mortality after $1 \mathrm{~h}$

Effective $-100 \%$ mortality

Effective $-100 \%$ mortality after $24 \mathrm{~h}$

Not effective $-<50 \%$ mortality

Not effective $-<50 \%$ mortality

Not effective $-<50 \%$ mortality

Partially effective $->50 \%$ mortality

Effective $-100 \%$ mortality after $1 \mathrm{~h}$

Partially effective $->50 \%$ mortality

Partially effective $->50 \%$ mortality

'Effective' - no details

'Effective' - no trophonts on treated fish but toxic to fish experiment stopped on $\mathrm{d} 9$
Wahli et al. (1993)

Buchman et al. (2003)

Wahli et al. (1993)

Buchman et al. (2003)

Wahli et al. (1993)

Buchman et al. (2003)

Wahli et al. (1993)

an and Goodwin 


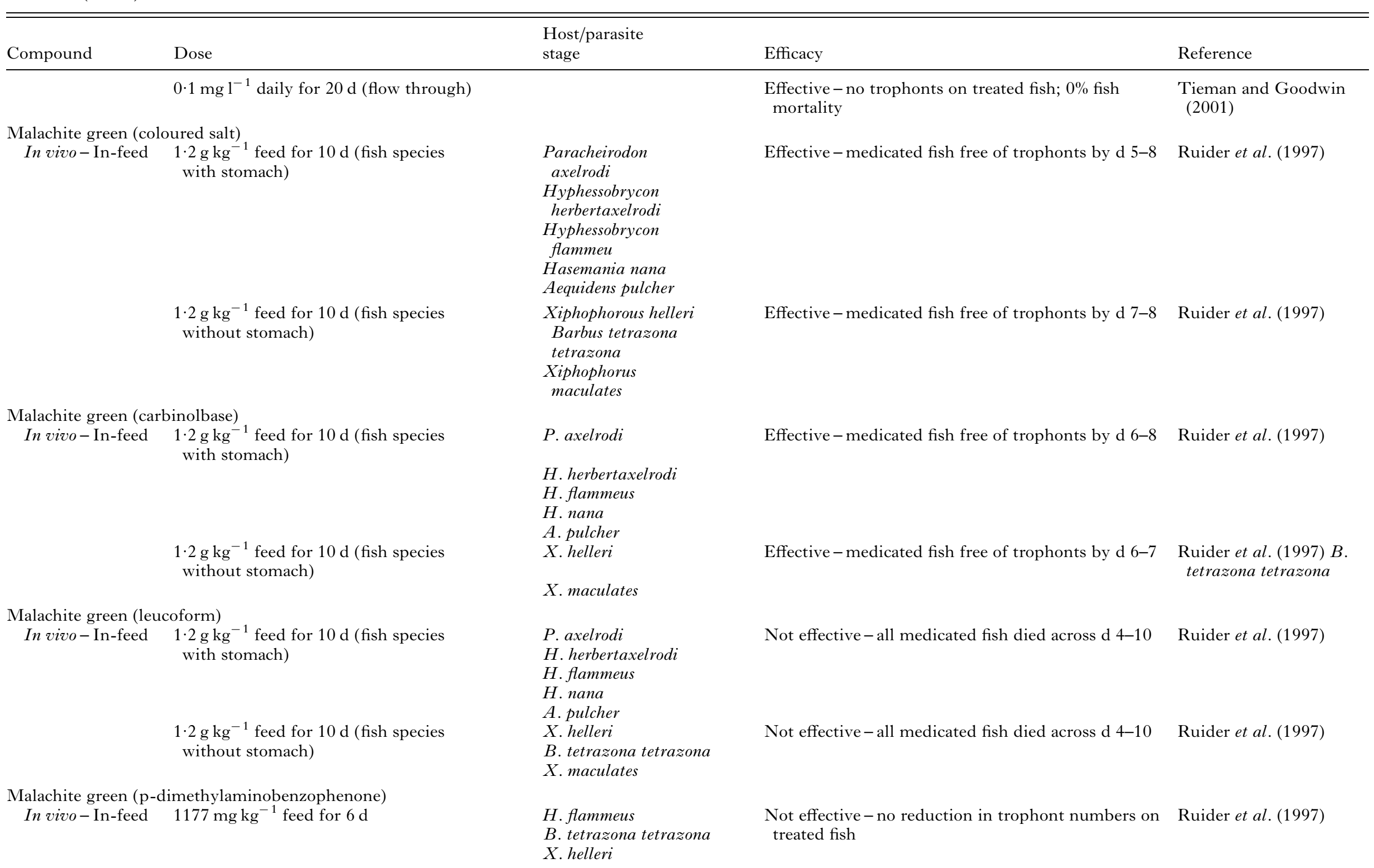


Malachite green (N,N-dimethylaniline)

In vivo-In-feed $316 \mathrm{mg} \mathrm{kg}^{-1}$ feed for $4 \mathrm{~d}$

H. nana

$X$. heller

X. maculates

Methylene blue

In vitro

In vivo-bath

$100 \mathrm{mg} 1^{-1}$ for $2 \mathrm{~min}$

$2 \mathrm{mg} \mathrm{l}^{-1}$ daily for $20 \mathrm{~d}$

Protomonts

I. punctatus

Alternate days for $20 \mathrm{~d}: 2 \mathrm{mg} \mathrm{l}^{-1}$ and

$100 \mathrm{mg} \mathrm{l}^{-1}$ formaldehyde (static tanks)

\section{Metronidazole \\ In vitro \\ $25 \mathrm{mg} \mathrm{l}^{-1}$ for $3 \mathrm{~h}$ \\ $25 \mathrm{mg} \mathrm{l}^{-1}$ for $24 \mathrm{~h}$ \\ In vivo - In-feed \\ $7 \cdot 5 \mathrm{mg} \mathrm{kg}^{-1}$ b.w for $7 \mathrm{~d}$ \\ $24 \mathrm{mg} \mathrm{kg}^{-1}$ b.w for $10 \mathrm{~d}$ \\ $36 \mathrm{mg} \mathrm{kg}^{-1}$ b.w for $10 \mathrm{~d}$ \\ $40 \mathrm{~g} \mathrm{~kg}^{-1}$ feed for $10 \mathrm{~d}$}

$200 \mathrm{mg} \mathrm{l}^{-1}$ for $2 \mathrm{~h}$

Protomonts

In vitro

$200 \mathrm{mg} \mathrm{l}^{-1}$ for $6 \mathrm{~d}$

O. mykiss

In vivo-bath

In-feed

Theronts
Adults**
Tomocysts
Salmonids not
specified
C. auratus
O. mykiss

O. mykiss
Not effective - no reduction in trophont numbers on Ruider et al. (1997) treated fish

Effective $-100 \%$ mortality after $12 \mathrm{~h}$ mortality $\dagger$

Not effective - no trophonts on treated fish but

$40-70 \%$ fish mortality

Not effective - no details

Not effective - no details

Not effective - no details

'Effective' - no details

Effective - no trophonts on treated fish

Effective - no trophonts on treated fish

Not effective $-35 \%$ of medicated fish were free of infection

Not effective $-12 \cdot 5 \%$ mortality after $2 \mathrm{~h}$; surviving protomonts developed normally

Not effective - all trophonts developed normally

Not effective - all trophonts developed normally

Not effective - all medicated fish had high numbers of Tojo-Rodriguez and

O. mykiss trophonts

MinnFinn $^{\mathrm{TM}}$

See entry for peracetic Acid (PAA) + acetic acid + hydrogen peroxide based formulation $\left(4 \cdot 5 \%\right.$ PAA $+9 \%$ AA $\left.+22 \% \mathrm{H}_{2} \mathrm{O}_{2}\right)$

Monensin sodium based formulation sold as commercial product as Elancoban

In vitro

$\begin{array}{ll}20 \mathrm{mg} \mathrm{l}^{-1} \text { for } 1 \mathrm{~h} & \text { Trophonts* } \\ & \\ 50 \mathrm{mg} \mathrm{l}^{-1} \text { for } 1 \mathrm{~h} & \\ 100 \mathrm{mg} \mathrm{l}^{-1} \text { for } 1 \mathrm{~h} & \text { Trophonts* } \\ 20 \mathrm{mg} \mathrm{l}^{-1} \text { for } 1 \mathrm{~h} & \text { Theronts }\end{array}$

$20 \mathrm{mg} \mathrm{l}^{-1}$ for $1 \mathrm{~h}$

Theronts

$50 \mathrm{mg} \mathrm{l}^{-1}$ for $1 \mathrm{~h}$

$100 \mathrm{mg} \mathrm{l}^{-1}$ for $1 \mathrm{~h}$

In vivo-In-feed $2 \mathrm{mg} \mathrm{kg}^{-1}$ feed for $10 \mathrm{~d}$

$5 \mathrm{mg} \mathrm{kg}^{-1}$ feed for $10 \mathrm{~d}$
Effective $-100 \%$ mortality after $14 \cdot 5 \mathrm{~h}$ of exposure

Effective $-100 \%$ mortality after $14 \cdot 5 \mathrm{~h}$ of exposure Effective $-100 \%$ mortality

Not effective $-27 \cdot 4 \%$ mortality

Not effective $-36 \cdot 8 \%$ mortality

Partially effective $-48 \cdot 2 \%$ mortality

Not effective - no significant reduction in trophont numbers

Not effective-no significant reduction in trophont numbers
Post and Vesely (1983)

(2001)

Tieman and Goodwin 2001)

Wahli et al. (1993)

Rahkonen and Koski (2002)

Tokşen and Nemli (2010)

Tojo-Rodriguez and Santamarina-Fernandez (2001)

Tojo-Rodriguez et al. (1994)

Tojo-Rodriguez et al (1994)

Santamarina-Fernandez (2001)

Shinn et al.

(unpublished)

Shinn et al. (2001)

Shinn et al.

(unpublished)

Shinn et al. (2001)

Shinn et al.

(unpublished) 


\begin{tabular}{|c|c|c|c|c|}
\hline Compound & Dose & $\begin{array}{l}\text { Host/parasite } \\
\text { stage }\end{array}$ & Efficacy & Reference \\
\hline & $10 \mathrm{mg} \mathrm{kg}^{-1}$ feed for $10 \mathrm{~d}$ & & $\begin{array}{l}\text { Not effective - no significant reduction in trophont } \\
\text { numbers }\end{array}$ & \\
\hline & $100 \mathrm{mg} \mathrm{kg}^{-1}$ feed for $10 \mathrm{~d}$ prior to inf. & O. mykiss & Not effective - no reduction in trophont numbers & Shinn et al. (2003a) \\
\hline \multicolumn{5}{|c|}{ Mucuna pruriens (velvet bean) } \\
\hline In vitro & $\begin{array}{l}100 \mathrm{mg} \mathrm{l}^{-1} \text { for } 3 \mathrm{~h} \\
100 \mathrm{mg} \mathrm{l}^{-1} \text { for } 6 \mathrm{~h} \\
150 \mathrm{mg} \mathrm{l}^{-1} \text { for } 3 \mathrm{~h} \\
150 \mathrm{mg} \mathrm{l}^{-1} \text { for } 6 \mathrm{~h} \\
200 \mathrm{mg} \mathrm{l}^{-1} \text { for } 3 \mathrm{~h} \\
200 \mathrm{mg} \mathrm{l}^{-1} \text { for } 6 \mathrm{~h}\end{array}$ & Trophonts* & $\begin{array}{l}\text { Not effective }-0 \% \text { mortality } \\
\text { Partially effective }-65 \% \text { mortality } \\
\text { Not effective }-25 \% \text { mortality } \\
\text { Effective }-100 \% \text { mortality } \\
\text { Not effective }-35 \% \text { mortality } \\
\text { Effective }-100 \% \text { mortality }\end{array}$ & Ekamen et al. (2004) \\
\hline \multirow[t]{3}{*}{ In vivo-bath } & $100 \mathrm{mg} \mathrm{l}^{-1}$ for $72 \mathrm{~h}$ & C. a. auratus & $\begin{array}{l}\text { Partially effective }-59 \% \text { reduction on the skin } / 60 \% \text { on } \\
\text { the gills }\end{array}$ & Ekamen et al. (2004) \\
\hline & $150 \mathrm{mg} \mathrm{l}^{-1}$ for $72 \mathrm{~h}$ & & $\begin{array}{l}\text { Effective }-79 \% \text { reduction in trophonts on the skin/ } \\
83 \% \text { on the gills }\end{array}$ & \\
\hline & $200 \mathrm{mg} \mathrm{l}^{-1}$ for $72 \mathrm{~h}$ & & $\begin{array}{l}\text { Effective }-92 \% \text { reduction in trophonts on the skin/ } \\
91 \% \text { on the gills }\end{array}$ & \\
\hline \multicolumn{5}{|l|}{ Na-desoxycholate } \\
\hline In vitro & $25 \mathrm{mg} \mathrm{l}^{-1}$ for $3 \mathrm{~h}$ & Theronts & Not effective - no details & Wahli et al. (1993) \\
\hline & $25 \mathrm{mg} \mathrm{l}^{-1}$ for $24 \mathrm{~h}$ & Adults** & Not effective - no details & \\
\hline & $25 \mathrm{mg} \mathrm{l}^{-1}$ for $24 \mathrm{~h}$ & Tomoysts & Not effective - no details & \\
\hline \multicolumn{5}{|l|}{ Netobimin } \\
\hline In vivo - In-feed & $40 \mathrm{~g} \mathrm{~kg}^{-1}$ feed for $10 \mathrm{~d}$ & O. mykiss & $\begin{array}{l}\text { Partially effective }-75 \% \text { of medicated fish with } \\
\text { moderate number of trophonts }\end{array}$ & $\begin{array}{l}\text { Tojo-Rodriguez and } \\
\text { Santamarina-Fernandez } \\
\text { (2001) }\end{array}$ \\
\hline \multicolumn{5}{|l|}{ Nicarbazin } \\
\hline In vivo - In-feed & $100 \mathrm{mg} \mathrm{kg}^{-1}$ feed for $10 \mathrm{~d}$ prior inf. & O. mykiss & $\begin{array}{l}\text { Not effective-no reduction in trophont numbers on } \\
\text { treated fish }\end{array}$ & Shinn et al. (2003a) \\
\hline \multicolumn{5}{|l|}{ Niridazole } \\
\hline In vivo-In-feed & $40 \mathrm{~g} \mathrm{~kg}^{-1}$ feed for $10 \mathrm{~d}$ & O. mykiss & $\begin{array}{l}\text { Effective }-90 \% \text { of medicated fish with low numbers } \\
\text { of trophonts }\end{array}$ & $\begin{array}{l}\text { Tojo-Rodriguez and } \\
\text { Santamarina-Fernandez } \\
\text { (2001) }\end{array}$ \\
\hline \multicolumn{5}{|l|}{ Nitroscanate } \\
\hline In vivo-In-feed & $40 \mathrm{~g} \mathrm{~kg}^{-1}$ feed for $10 \mathrm{~d}$ & O. mykiss & $\begin{array}{l}\text { Partially effective }-50 \% \text { of medicated fish had no } \\
\text { trophonts }\end{array}$ & $\begin{array}{l}\text { Tojo-Rodriguez and } \\
\text { Santamarina-Fernandez } \\
\text { (2001) }\end{array}$ \\
\hline \multicolumn{5}{|l|}{ N-methylglucamine } \\
\hline In vivo - In-feed & $40 \mathrm{~g} \mathrm{~kg}^{-1}$ feed for $10 \mathrm{~d}$ & O. mykiss & $\begin{array}{l}\text { Not effective - all medicated fish had high numbers of } \\
\text { trophonts }\end{array}$ & $\begin{array}{l}\text { Tojo-Rodriguez and } \\
\text { Santamarina-Fernandez } \\
(2001)\end{array}$ \\
\hline
\end{tabular}


Ornidazole

In vivo-In-feed $24 \mathrm{mg} \mathrm{kg}^{-1} \mathrm{~b} . \mathrm{w}$ for $10 \mathrm{~d}$ $36 \mathrm{mg} \mathrm{kg}^{-1}$ b.w for $10 \mathrm{~d}$

Effective - no trophonts on the treated groups Effective - no trophonts on the treated group

Oxytetracycline

In vitro

$100 \mathrm{mg}^{-1}$ for $3 \mathrm{~h}$

$100 \mathrm{mg} \mathrm{l}^{-1}$ for $24 \mathrm{~h}$

$100 \mathrm{mg} \mathrm{l}^{-1}$ for $24 \mathrm{~h}$

$200 \mathrm{mg} \mathrm{l}^{-1}$ for $2 \mathrm{~h}$

Pamomy

In vitro

In vivo-bath

$200 \mathrm{mg} \mathrm{l}^{-1}$ for $3 \mathrm{~h}$

$1000 \mathrm{mg} \mathrm{kg}^{-1}$ for $8 \mathrm{~d}$

In-feed

Peracetic acid (PAA) + acetic acid + hydrog
In vitro $0.4 \mathrm{mg} \mathrm{l}^{-1}$ for $48 \mathrm{~h}$ $0.5 \mathrm{mg} \mathrm{l}^{-1}$ for $48 \mathrm{~h}$

$0.6 \mathrm{mg} \mathrm{l}^{-1}$ for $48 \mathrm{~h}$

$0.7 \mathrm{mg} \mathrm{l}^{-1}$ for $48 \mathrm{~h}$

$0 \cdot 8 \mathrm{mg} \mathrm{l}^{-1}$ for $48 \mathrm{~h}$

$0.9 \mathrm{mg} \mathrm{l}^{-1}$ for $48 \mathrm{~h}$

$0 \cdot 5 \mathrm{mg} \mathrm{l}^{-1}$ for $12 \mathrm{~h}$

$1 \mathrm{mg} \mathrm{l}^{-1}$ for $12 \mathrm{~h}$

$2 \mathrm{mg} \mathrm{l}^{-1}$ for $12 \mathrm{~h}$

$3 \mathrm{mg} \mathrm{l}^{-1}$ for $12 \mathrm{~h}$

$0 \cdot 5 \mathrm{mg} \mathrm{l}^{-1}$ for $2 \mathrm{~h}$

$0 \cdot 5 \mathrm{mg} \mathrm{l}^{-1}$ for $4 \mathrm{~h}$

$1 \mathrm{mg} \mathrm{l}^{-1}$ for $2 \mathrm{~h}$

$1 \mathrm{mg} \mathrm{l}^{-1}$ for $4 \mathrm{~h}$

$2 \mathrm{mg} \mathrm{l}^{-1}$ for $2 \mathrm{~h}$

$2 \mathrm{mg} \mathrm{l}^{-1}$ for $4 \mathrm{~h}$

$2 \cdot 5 \mathrm{mg} \mathrm{l}^{-1}$ for $2 \mathrm{~h}$

$3 \mathrm{mg} \mathrm{l}^{-1}$ for $2 \mathrm{~h}$

$2.5 \mathrm{mg} \mathrm{l}^{-1}$ for $4 \mathrm{~h}$

$0.04 \mathrm{mg} \mathrm{l}^{-1}$ for $1-4 \mathrm{~h}$

$0.08 \mathrm{mg}^{-1}$ for $1-4 \mathrm{~h}$

$0 \cdot 12 \mathrm{mg} \mathrm{l}^{-1}$ for $1-4 \mathrm{~h}$

$0 \cdot 16 \mathrm{mg} \mathrm{l}^{-1}$ for $1-3 \mathrm{~h}$

$0 \cdot 16 \mathrm{mg} \mathrm{l}^{-1}$ for $4 \mathrm{~h}$

$0 \cdot 20 \mathrm{mg} \mathrm{l}^{-1}$ for $1 \mathrm{~h}$

$0 \cdot 20 \mathrm{mg} \mathrm{l}^{-1}$ for $1-4 \mathrm{~h}$

$0 \cdot 20 \mathrm{mg} \mathrm{l}^{-1}$ for $2-4 \mathrm{~h}$

Effective $-82 \%$ mortality
Theronts

Adults**

Tomocysts

O. mykiss

Not effective - medium survival rate after $3 \mathrm{~h}$

Not effective-high survival rate after $24 \mathrm{~h}$

Not effective-high survival rate after $24 \mathrm{~h}$

Partially effective $-75 \%$ mortality after $2 \mathrm{~h}$, surviving protomonts reproduce normally

Not effective - all trophonts developed normally

Not effective - all trophonts developed normally

$\mathrm{H}_{2} \mathrm{O}_{2}$ ) sold as commercial product as Wofasteril ${ }^{\circledR}$

Not effective $-21 \%$ mortality

Not effective $-20 \%$ mortality

Not effective $-39 \%$ mortality

Not effective- $\sim 40 \%$ mortality

Partially effective $-\sim 75 \%$ mortality

Tomocysts $(<2 \cdot 5 \mathrm{~h})$

Not effective $-42 \%$ mortality

Partially effective $-75 \%$ mortality

Effective $-98 \%$ mortality

Effective - > $99 \%$ mortality

Tomocysts $(24 \mathrm{~h}+)$

Not effective - all trophonts developed normally Not effective - all trophonts developed normally Not effective - all trophonts developed normally Not effective-all trophonts developed normally

Not effective - all trophonts developed normally

Not effective - all trophonts developed norm

Not effective - all trophonts developed normally

Not effective - all trophonts developed normally

Not effective - all trophonts developed normally

Theronts

Not effective $-0-5 \%$ mortality

Not effective $-0-5 \%$ (trial 1 ); $10-20 \%$ mortality (tria

Not effective $-5-10 \%$ (trial 1); $20-30 \%$ mortality (trial 2)

Not effective

Not effective $-40 \%$ mortality

Not effective $-30 \%$ mortality

Partially effective $-50 \%$ mortality
Tojo-Rodriguez et al (1994)

Tokşen and Nemli (2010)

Wahli et al. (1993)

(1994)

Meinelt et al. (2009)

Meinelt et al. (2009)

Straus and Meinelt (2009)

Meinelt et al. (2009) 


\begin{tabular}{ll}
\hline \hline Compound & Dose \\
\hline & $0.24 \mathrm{mg} \mathrm{l}^{-1}$ for $1-4 \mathrm{~h}$ \\
& $0.28 \mathrm{mg} \mathrm{l}^{-1}$ for $1-4 \mathrm{~h}$ \\
& $0.32 \mathrm{mg} \mathrm{l}^{-1}$ for $1-4 \mathrm{~h}$ \\
& $0.36 \mathrm{mg} \mathrm{l}^{-1}$ for $1-4 \mathrm{~h}$ \\
& $0.40 \mathrm{mg} \mathrm{l}^{-1}$ for $1-4 \mathrm{~h}$
\end{tabular}

Host/parasite

stage

Efficacy

Reference

In vivo - bath ( $40 \%$ PAA solution)

$1 \mathrm{mg} \mathrm{l}^{-1}$ for $4 \mathrm{~d}$

C. carpio

Not effective $-30 \%$ mortality (trial 1); $60 \%$ mortality

(trial 2)

Partially effective $-50 \%$ mortality (trial 1 ); $70-80 \%$ mortality (trial 2)

Effective $-60 \%$ mortality (trial 1); 80-90\% mortality (trial 2)

Effective $-70 \%$ mortality (trial 1 ); $80-95 \%$ mortality (trial 2)

Effective $-80 \%$ mortality (trial 1); $90-95 \%$ mortality

(trial 2)

Effective - significant reduction on the number of trophonts on treated fish

Peracetic Acid (PAA) + hydrogen peroxide + based formulation (4.5\% PAA + 22\% $\mathrm{H}_{2} \mathrm{O}_{2}+9 \%$ AA) sold as the commercial product as MinnFinn ${ }^{\mathrm{TM}}$ In vitro

(theronts from Notemigonus crysoleucas)

$0 \cdot 0225 \mathrm{mg} \mathrm{l}^{-1}$ for $1-4 \mathrm{~h}$

Not effective $-0 \%$ mortality

Straus and Meinelt

$0 \cdot 0450 \mathrm{mg} \mathrm{l}^{-1}$ for $1-4 \mathrm{~h}$

$0.0675 \mathrm{mg} \mathrm{l}^{-1}$ for $1-4 \mathrm{~h}$

$0.0900 \mathrm{mg} \mathrm{l}^{-1}$ for $1-4 \mathrm{~h}$

$0 \cdot 1125 \mathrm{mg} \mathrm{l}^{-1}$ for $1-4 \mathrm{~h}$

$0 \cdot 1350 \mathrm{mg} \mathrm{l}^{-1}$ for $1 \mathrm{~h}$

$0 \cdot 1350 \mathrm{mg} \mathrm{l}^{-1}$ for $2-4 \mathrm{~h}$

$0 \cdot 1575 \mathrm{mg} \mathrm{l}^{-1}$ for $1 \mathrm{~h}$

$0 \cdot 1575 \mathrm{mg} \mathrm{l}^{-1}$ for $2-4 \mathrm{~h}$

$0 \cdot 1800 \mathrm{mg} \mathrm{l}^{-1}$ for $1 \mathrm{~h}$

$0 \cdot 1800 \mathrm{mg} \mathrm{l}^{-1}$ for $2-4 \mathrm{~h}$

$0.2025 \mathrm{mg} \mathrm{l}^{-1}$ for $1 \mathrm{~h}$

$0 \cdot 2025 \mathrm{mg} \mathrm{l}^{-1}$ for $2-4 \mathrm{~h}$

$0 \cdot 2250 \mathrm{mg} \mathrm{l}^{-1}$ for $1-4 \mathrm{~h}$

(theronts from Xiphophorus hellerii)

$0.0225 \mathrm{mg}^{-1}$ for $1-4 \mathrm{~h}$

$0.0450 \mathrm{mg} \mathrm{l}^{-1}$ for $1-4 \mathrm{~h}$

$0.0675 \mathrm{mg} \mathrm{l}^{-1}$ for $1-4 \mathrm{~h}$

$0.0900 \mathrm{mg} \mathrm{l}^{-1}$ for $1-4 \mathrm{~h}$

$0 \cdot 1125 \mathrm{mg} \mathrm{l}^{-1}$ for $1-3 \mathrm{~h}$

$0 \cdot 1125 \mathrm{mg} \mathrm{l}^{-1}$ for $4 \mathrm{~h}$

$0 \cdot 1350 \mathrm{mg} \mathrm{l}^{-1}$ for $1 \mathrm{~h}$

$0 \cdot 1350 \mathrm{mg} \mathrm{l}^{-1}$ for $2-4 \mathrm{~h}$

$0 \cdot 1575 \mathrm{mg} \mathrm{l}^{-1}$ for $1-4 \mathrm{~h}$

Not effective $-0 \%$ mortality

(2009)

$0.1800 \mathrm{mg} \mathrm{l}^{-1}$

Not effective $-5 \%$ mortality

Not effective $-5-20 \%$ mortality

Not effective $-20-40 \%$ mortality

Not effective $-30 \%$ mortality

Partially effective $-50 \%$ mortality

Not effective $-40 \%$ mortality

Partially effective $-60 \%$ mortality

Not effective $-40 \%$ mortality

Partially effective $-60 \%$ mortality

Not effective $-40 \%$ mortality

Partially effective $-70 \%$ mortality

Partially effective $-50-70 \%$ mortality

Not effective $-0-10 \%$ mortality

Not effective $-10 \%$ mortality

Not effective $-10-20 \%$ mortality

Not effective $-10-30 \%$ mortality

Not effective $-20-40 \%$ mortality

Partially effective $-50 \%$ mortality

Not effective $-40 \%$ mortality

Partially effective $-50 \%$ mortality

Partially effective $-50-60 \%$ mortality

Effective $-60-80 \%$ mortality 
Peracetic acid + acetic acid + hydrogen peroxide based formulation sold as the commercial product Detarox

In vivo-bath $10 \mathrm{mg} \mathrm{l}^{-1}$ for $25-45 \mathrm{~min} 2 \mathrm{nd}$ treatment Salmonids - not specified 'Effective' - no details after $4-7 \mathrm{~d}$

Rhakonen and Koski after $4-7$

Peracetic acid + acetic acid + hydrogen peroxide based formulation (13\% PAA $+20 \%$ AA $\left.+20 \% \mathrm{H}_{2} \mathrm{O}_{2}\right)$ sold as commercial product Per Aqua
In vitro

$0.08 \mathrm{mg}^{-1}$ for $30 \mathrm{~min}$ up to $60 \mathrm{~min}$

Protomonts

Not effective $-0-20 \%$ mortality

$0.08 \mathrm{mg} \mathrm{l}^{-1}$ for $1.5 \mathrm{~h}$ up to $4.5 \mathrm{~h}$

Not effective $-30-40 \%$ mortality

Bruzio and Buchmann

$0 \cdot 8 \mathrm{mg} \mathrm{l}^{-1}$ for $15 \mathrm{~min}$

Not effective $-20 \%$ mortality

$0.8 \mathrm{mg} \mathrm{l}^{-1}$ for $30 \mathrm{~min}$

Effective - 90\% mortality

$0.8 \mathrm{mg} \mathrm{l}^{-1}$ for $45 \mathrm{~min}$

$0.8 \mathrm{mg} \mathrm{l}^{-1}$ for $15 \mathrm{~min}$

Effective - 100\% mortality

$8 \mathrm{mg} \mathrm{l}^{-1}$ for $15 \mathrm{~min}$

Effective $-100 \%$ mortality

In vivo-bath

$40 \mathrm{mg} \mathrm{l}^{-1} 3$ times a week for 3 weeks

Theronts

Effective $-100 \%$ mortalit

Peracetic acid + acetic acid + hydrogen peroxide based formulation $\left(13 \%\right.$ PAA $+20 \%$ AA $\left.+20 \% \mathrm{H}_{2} \mathrm{O}_{2}\right)$ sold as commercial product Desirox + formaldehyde

In vivo-bath $\quad 10+100 \mathrm{mg} \mathrm{l}^{-1} 3$ times a week $\quad$ S. salar $\quad$ Trial inconclusive-details missing

Rintamäki-Kinnunen et al. $(2005 a)$

Rintamäki-Kinnunen for 4 weeks

formulation sold as commercial product Incimaxx Aquatic

et al. $(2005 a)$

Peracetic acid + acetic acid + hydrogen peroxide + peroctanoic acid based formulation sold as commercial product Incimaxx Aquatic

$\begin{array}{lll}\text { In vitro } & 0.08 \mathrm{mg} \mathrm{l}^{-1} \text { for } 30 \mathrm{~min} \text { up to } 4.5 \mathrm{~h} & \text { Protomonts } \\ 0.8 \mathrm{mg} \mathrm{l}^{-1} \text { for } 15 \mathrm{~min} & \text { Not effective }-\sim 15-20 \% \text { mortal }\end{array}$

Effective $-80 \%$ mortality

$0 \cdot 8 \mathrm{mg} \mathrm{l}^{-1}$ for $30 \mathrm{~min}$
$0 \cdot 8 \mathrm{mg} \mathrm{l}^{-1}$ for $45 \mathrm{~min}$

Effective $-80 \%$ mortality
Effective $-100 \%$ mortality

$8 \mathrm{mg} \mathrm{l}^{-1}$ for $1 \mathrm{~h} \quad$ Effective $-100 \%$ mortality

Effective $-100 \%$ mortality

$12 \mathrm{mg} \mathrm{l}^{-1}$ for $1 \mathrm{~h}$

$15 \mathrm{mg} \mathrm{l}^{-1}$ for $1 \mathrm{~h}$

Effective $-100 \%$ mortality

$8 \mathrm{mg} \mathrm{l}^{-1}$ for $1 \mathrm{~h}$

Tomocysts

Effective $-100 \%$ mortality

$12 \mathrm{mg} \mathrm{l}^{-1}$ for $1 \mathrm{~h}$
$15 \mathrm{mg} \mathrm{l}^{-1}$ for $1 \mathrm{~h}$

$0 \cdot 8 \mathrm{mg} \mathrm{l}^{-1}$ for $15 \mathrm{~min}$

Effective $-100 \%$ mortality

Effective $-100 \%$ mortality

$8 \mathrm{mg} \mathrm{l}^{-1}$ for $15 \mathrm{~min}$

Effective $-100 \%$ mortality

$8 \mathrm{mg} \mathrm{l}^{-1}$ for $1 \mathrm{~h}$

Effective $-100 \%$ mortality

$12 \mathrm{mg} \mathrm{l}^{-1}$ for $1 \mathrm{~h}$

Effective $-98 \cdot 3 \%$ mortality

Effective $-100 \%$ mortality

$15 \mathrm{mg} \mathrm{l}^{-1}$ for $1 \mathrm{~h}$

Effective $-100 \%$ mortality

Bruzio and Buchmann (2010)

Picón-Camacho et al. (in press $b$ )

Picón-Camacho et al. (in press $b$ )

Bruzio and Buchmann (2010)

Picón-Camacho et al.

(in press $b$ )

Per Aqua

See entry for peracetic acid + acetic acid + hydrogen peroxide based formulations $\left(13 \%\right.$ PAA $\left.+20 \% A A+20 \% \mathrm{H}_{2} \mathrm{O}_{2}\right)$

Perotan

See entry for hydrogen peroxide + acetic acid based formulations 


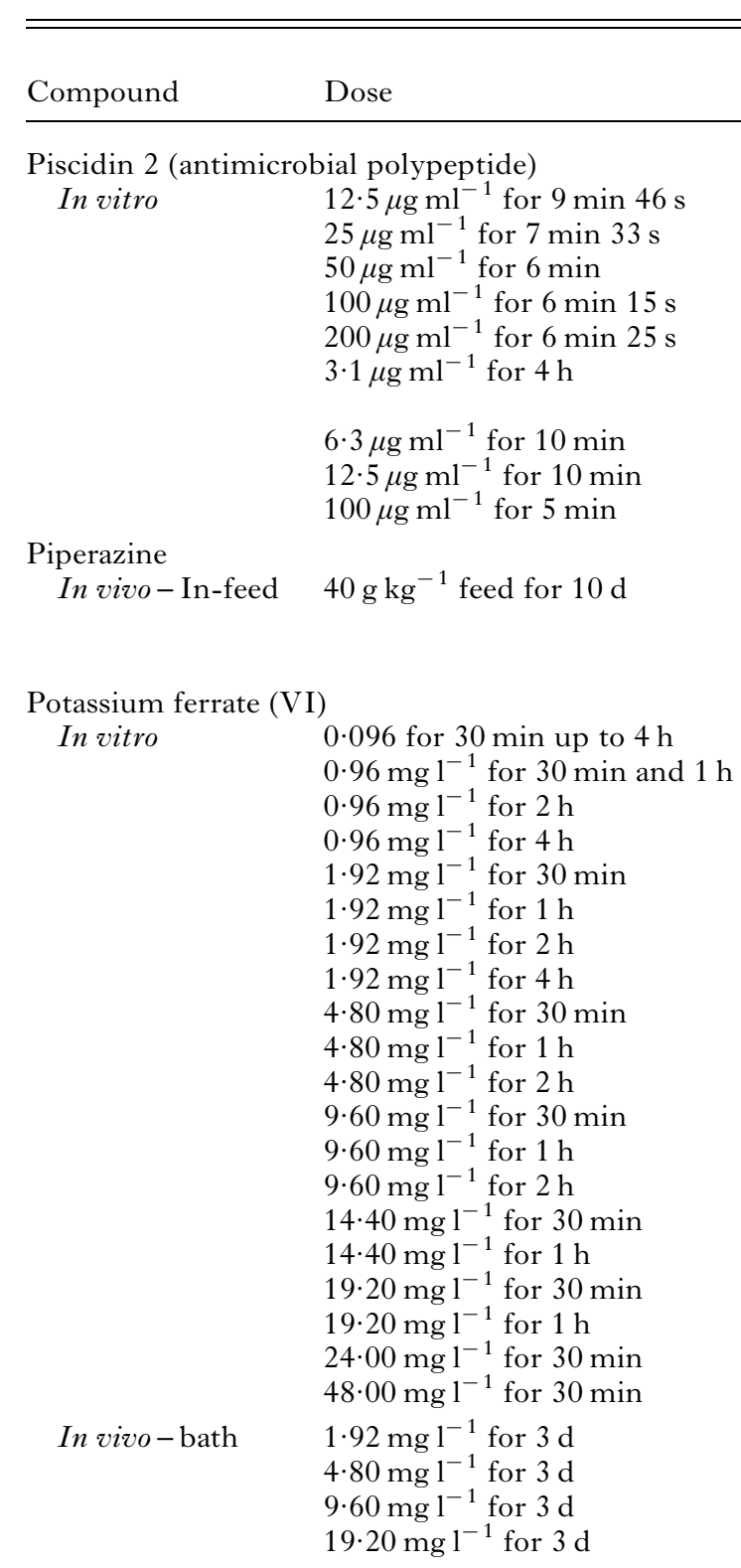

Host/parasite
stage

Efficacy

Reference

Trophonts*

Theronts

$$
\begin{aligned}
& 6 \cdot 3 \mu \mathrm{g} \mathrm{ml}^{-1} \text { for } 10 \mathrm{~min} \\
& 12 \cdot 5 \mu \mathrm{g} \mathrm{ml}^{-1} \text { for } 10 \mathrm{~min} \\
& 100 \mu \mathrm{g} \mathrm{ml}^{-1} \text { for } 5 \mathrm{~min}
\end{aligned}
$$

Piperazine

O. mykiss

Theronts

Effective $-100 \%$ mortality

Effective $-100 \%$ mortality

Effective $-100 \%$ mortality

Effective $-100 \%$ mortality

Effective $-100 \%$ mortality

Not effective $-0 \%$ killed, but less active than those in Colorni et al. (2008) the controls

Effective $-100 \%$ mortality

Effective $-100 \%$ mortality

Effective $-100 \%$ mortality

Partially effective $-55 \%$ of the medicated fish had a low number of trophonts

Tojo-Rodriguez and Santamarina-Fernandez (2001)

Not effective $-0 \%$ mortality

Not effective $-0 \%$ mortality

Not effective $-14 \%$ mortality

Not effective $-25 \%$ mortality

Not effective $-0 \%$ mortality

Not effective $-20 \%$ mortality

Not effective $-24 \%$ mortality

Partially effective $-56 \%$ mortality

Not effective $-38 \%$ mortality

Partially effective $-58 \%$ mortality

Effective $-100 \%$ mortality

Partially effective $-50 \%$ mortality

Partially effective $-64 \%$ mortality

Effective $-100 \%$ mortality

Partially effective $-59 \%$ mortality

Effective $-100 \%$ mortality

Partially effective $-69 \%$ mortality

Effective $-100 \%$ mortality

Effective $-100 \%$ mortality

Effective $-100 \%$ mortality

C. auratus

Partially effective $-71.94 \%$ trophont reduction

Effective $-80 \cdot 30 \%$ trophont reduction

Effective $-83 \cdot 39 \%$ trophont reduction

Effective $-100 \%$ trophont reduction
Ling et al. (2010)

Ling et al. (2010) 
Potassium permanganate $\left(\mathrm{KMnO}_{4}\right)$

In vitro $\quad 0 \cdot 1-0.5 \mathrm{mg} \mathrm{l}^{-1}$ for 15 up to $45 \mathrm{~min}$

$0.6 \mathrm{mg} \mathrm{l}^{-1}$ for $15 \mathrm{~min}$ up to $4 \mathrm{~h}$

$0.7 \mathrm{mg} \mathrm{l}^{-1}$ for $15 \mathrm{~min}$ up to $4 \mathrm{~h}$

$0.8 \mathrm{mg} \mathrm{l}^{-1}$ for $15 \mathrm{~min}$

$0 \cdot 8 \mathrm{mg} \mathrm{l}^{-1}$ for $30 \mathrm{~min}$ up to $4 \mathrm{~h}$

$0.9 \mathrm{mg} \mathrm{l}^{-1}$ for $15 \mathrm{~min}$

$0.9 \mathrm{mg} \mathrm{l}^{-1}$ for $30 \mathrm{~min}$ up to $4 \mathrm{~h}$

$1.0 \mathrm{mg} \mathrm{l}^{-1}$ for $15 \mathrm{~min}$

$1.0 \mathrm{mg} \mathrm{l}^{-1}$ for $30 \mathrm{~min}$ up to $4 \mathrm{~h}$

In vivo-bath

$0 \cdot 25 \mathrm{mg}^{-1}$ for $6 \mathrm{~d}$

$0 \cdot 50 \mathrm{mg} \mathrm{l}^{-1}$ for $6 \mathrm{~d}$

$0 \cdot 75 \mathrm{mg}^{-1}$ for $6 \mathrm{~d}$

$1 \cdot 0 \mathrm{mg} \mathrm{l}^{-1}$ for $6 \mathrm{~d}$

$2 \mathrm{mg} \mathrm{l}^{-1}$ daily for $20 \mathrm{~d}$ (static tanks

and flow through)

$0 \cdot 25 \mathrm{mg} \mathrm{l}^{-1}$ for $8 \mathrm{~d}$

$0.50 \mathrm{mg} \mathrm{l}^{-1}$ for $8 \mathrm{~d}$

$0 \cdot 5 \mathrm{mg} \mathrm{l}^{-1}$ daily for $10 \mathrm{~d}$

$0.75 \mathrm{mg} \mathrm{l}^{-1}$ daily for $10 \mathrm{~d}$

$1.0 \mathrm{mg} \mathrm{l}^{-1}$ daily for $10 \mathrm{~d}$

$1 \cdot 25 \mathrm{mg} \mathrm{l}^{-1}$ daily for $10 \mathrm{~d}$

$1 \cdot 25 \mathrm{mg} \mathrm{l}^{-1}$ daily for $10 \mathrm{~d}$

$4 \mathrm{mg} \mathrm{l}^{-1} 3$ times a week for 2 weeks

S. salar

$10-20 \mathrm{mg} \mathrm{l}^{-1}$ for $30 \mathrm{~min}$

Potassium permanganate $\left(\mathrm{KMnO}_{4}\right)+$ dimetrazole

In vivo-bath $\quad 3 \mathrm{mg} \mathrm{l}^{-1}$ every $2 \mathrm{nd} \mathrm{d}$,

+ in-feed $\quad 5$ times + dimetrazole in feed

$28 \mathrm{mg} / \mathrm{fish}$ for $10 \mathrm{~d}$

Potassium persulfate + sodium dodecylbenzosulfonat

$1 \mathrm{mg} \mathrm{l}^{-1}$ for $1 \mathrm{~h}$

$10 \mathrm{mg} \mathrm{l}^{-1}$ for $1 \mathrm{~h}$

$100 \mathrm{mg} \mathrm{l}^{-1}$ for $1 \mathrm{~h}$
Theronts

I. punctatus

I. punctatus

Tilapia aurea

I. punctatus

O. mykiss

S. fontinalis

S. trutta

O. mykiss

Theronts

Trophonts*
Not effective - no details

Not effective $-0-1 \%$ mortality

Not effective $-5-10 \%$ mortality

Not effective $-0 \%$ mortality

Partially effective $-70 \%$ mortality

Not effective $-0 \%$ mortality

Effective $-90-95 \%$ mortality

Not effective $-0 \%$ mortality

Effective $-95->99 \%$ mortality

treated fish

Partially effective - low number of trophonts on

treated fish

Partially effective - low number of trophonts on

treated fish

Effective - No trophonts on treated fish

Not effective - all treated infected fish died; trophonts present

Effective - No trophonts on treated fish

Effective - No trophonts on treated fish

Not effective - high number of trophonts on treated fish

Not effective-high number of trophonts on treated fish

Effective - no trophonts on treated fish

Effective - no trophonts on treated fish

Not effective - toxic to treated fish

Trial inconclusive - low parasite numbers across all groups

'Effective' - there was a reduction in trophont

number on treated fish but was toxic to all three fish species

Effective - no signs of infection by $\mathrm{d} 7$

Rapp (1995)

Shinn et al. (2005)

Not effectir

Partially effective $-\sim 55 \%$ mortality

Not effective $-60 \%$ mortality

Straus and Griffin (2001)

Tieman and Goodwin (2001)

Straus and Griffin (2001)

Straus and Griffin (2002)

Rintamäki-Kinnunen

al. $(2005 a)$

Balta et al. (2008)

Not effective - trophonts develop normally
Lahnsteiner and

Weismann (2007) 
Pyceze $^{\mathrm{TM}}$

See entry for bronopol

Quinacrine

In vitro

$200 \mathrm{mg}^{-1}$ for $2 \mathrm{~h}$

In vivo-bath

$100 \mathrm{mg} \mathrm{l}^{-1}$ for $3 \mathrm{~h}$, d 6 p.i.

In-feed

$500 \mathrm{mg} \mathrm{kg}^{-1}$ for $8 \mathrm{~d}$

\section{Quinine}

$$
\begin{array}{ll}
\text { In vivo-In-feed } & 5 \mathrm{~g} \mathrm{~kg}^{-1} \text { feed for } 7 \mathrm{~d} \\
& 5 \mathrm{~g} \mathrm{~kg}^{-1} \text { feed for } 8 \mathrm{~d} \\
& 5 \mathrm{~g} \mathrm{~kg}^{-1} \text { feed for } 10 \mathrm{~d}
\end{array}
$$

1,3-di-6-quinolylurea

$$
\text { In vivo-In-feed } 40 \mathrm{~g} \mathrm{~kg}^{-1} \text { feed for } 10 \mathrm{~d}
$$

Ronidizole (1-methyl-2-carboxymethyl-5-nitroimidazole) In vitro

$$
\begin{aligned}
& 250 \mathrm{mg} \mathrm{l}^{-1} \text { for } 1 \mathrm{~h}-48 \mathrm{~h} \\
& 500 \mathrm{mg} \mathrm{l}^{-1} \text { for } 1 \mathrm{~h}-4 \mathrm{~h} \\
& 500 \mathrm{mg} \mathrm{l}^{-1} \text { for } 8 \mathrm{~h} \\
& 500 \mathrm{mg} \mathrm{l}^{-1} \text { for } 24 \mathrm{~h} \\
& 500 \mathrm{mg} \mathrm{l}^{-1} \text { for } 48 \mathrm{~h} \\
& 750 \mathrm{mg} \mathrm{l}^{-1} \text { for } 1 \mathrm{~h} \\
& 750 \mathrm{mg} \mathrm{l}^{-1} \text { for } 2 \mathrm{~h} \\
& 750 \mathrm{mg} \mathrm{l}^{-1} \text { for } 4 \mathrm{~h} \\
& 750 \mathrm{mg} \mathrm{l}^{-1} \text { for } 8 \mathrm{~h} \\
& 750 \mathrm{mg} \mathrm{l}^{-1} \text { for } 24 \mathrm{~h} \\
& 500 \mathrm{mg} \mathrm{l}^{-1} \text { for } 1 \mathrm{~h} \\
& 500 \mathrm{mg} \mathrm{l}^{-1} \text { for } 2 \mathrm{~h} \\
& 500 \mathrm{mg} \mathrm{l}^{-1} \text { for } 4,8 \text { and } 24 \mathrm{~h}
\end{aligned}
$$

Protomonts

Host/parasite

stage

O. mykiss

C. carpio

O. mykiss

Poecilia sphenops

$X$. heller

Hyphessobrycon

herbertaxelrodi

O. mykiss

Trophozites*

Tomites
Efficacy

Not effective - none of the treated fish survived

Not effective $-90 \%$ of the treated fish had survived on $\mathrm{d} 1 ; 50 \%$ were still alive by $\mathrm{d} 3$ but had a high number of trophonts

Partially effective $-50 \%$ mortality after $2 \mathrm{~h} ; 50 \%$ of protomonts develop normally

Not effective $-50 \%$ fish mortality; all trophonts developed normally

Not effective - all trophonts developed normally; signs of feed rejection from $\mathrm{d} 4$

Effective $-100 \%$ elimination of trophonts

Effective $-100 \%$ elimination of trophonts

Effective $-100 \%$ elimination of trophonts

Not effective - all treated fish had a high number of trophonts

Not effective $-0 \%$ mortality

Not effective $-0 \%$ mortality

Not effective $-25 \%$ mortality

Partially effective $-65 \cdot 5 \%$ mortality ( 3 repeat trials)

Partially effective $-65 \cdot 5 \%$ mortality

Not effective $-0 \%$ mortality

Not effective $-2 \cdot 5 \%$ mortality

Not effective $-47 \cdot 5 \%$ mortality

Effective $-82 \cdot 5 \%$ mortality

Effective $-87 \cdot 5 \%$ mortality (trial 1 ); $100 \%$ mortality (trial 2)

Not effective $-10 \%$ mortality

Farley and Heckmann (1980)

Not effective $-23 \%$ mortality

Effective $-55 \%, 90 \%$ and $96 \%$ mortality respectively
Tojo-Rodriguez et al. (1994)

Reference

Lahnsteiner and Weismann (2007)

Schmahl et al. (1996)

Tojo-Rodriguez and Santamarina-Fernandez (2001)

Farley and Heckmann (1980) 
$750 \mathrm{mg} \mathrm{l}^{-1}$ for $1 \mathrm{~h}$

$750 \mathrm{mg} \mathrm{l}^{-1}$ for $2,4,8$ and $24 \mathrm{~h}$

In vivo-In-feed

$40 \mathrm{~g} \mathrm{~kg}^{-1}$ feed for $10 \mathrm{~d}$

O. mykiss

SalarBec

$\begin{array}{cll}\text { In vivo-In-feed } & \begin{array}{c}0.32 \mathrm{mg} \mathrm{kg}^{-1} \text { feed for } 10 \mathrm{~d} \text { prior } \\ \text { infection }\end{array} & \text { O. mykiss } \\ \begin{array}{c}\text { Salinomycin sodium } \\ \text { In vivo-In-feed }\end{array} & \begin{array}{l}38 \mathrm{mg} \mathrm{kg}^{-1} \text { feed for } 10 \mathrm{~d} \mathrm{p} . \mathrm{i} . \\ 43 \mathrm{mg} \mathrm{kg}^{-1} \text { feed for } 10 \mathrm{~d} \text { p.i. } \\ 47 \mathrm{mg} \mathrm{kg}^{-1} \text { feed for } 10 \mathrm{~d} \text { p.i. }\end{array} & \text { O. mykiss } \\ \text { Secnidazole } & & \\ \text { In vivo-In-feed } & 24 \mathrm{mg} \mathrm{kg}^{-1} \text { b.w for } 10 \mathrm{~d} & \text { C. auratus } \\ & 36 \mathrm{mg} \mathrm{kg}^{-1} \text { b.w for } 10 \mathrm{~d} & \text { O. mykiss } \\ & 40 \mathrm{~g} \mathrm{~kg}^{-1} \text { feed for } 10 \mathrm{~d} & \end{array}$

Silver nitrate

In vitro

$0 \cdot 67 \mathrm{mg}^{-1}$

Tomites

Not specified

odium carbonate peroxyhydrate

In vivo - bath $\quad 60-90 \mathrm{mg} \mathrm{l}^{-1}$ daily for $30-1 \mathrm{~h}$ for 4-6 d

Sodium chloride $(\mathrm{NaCl})$

$\begin{array}{ll}\text { In vitro } & 2 \cdot 5 \mathrm{~g} \mathrm{l}^{-1} \text { for } 24 \mathrm{~h} \\ & 5 \mathrm{~g}^{-1} \text { for } 24 \mathrm{~h} \\ & 10 \mathrm{~g}^{-1} \text { for } 24 \mathrm{~h} \\ & 15 \mathrm{~g}^{-1} \text { for } 10 \mathrm{~h} \\ & 20 \mathrm{~g}^{-1} \text { for } 10 \mathrm{~h} \\ & 1 \mathrm{~g}^{-1} \text { for } 12 \mathrm{~d} \\ & 1 \mathrm{~g}^{-1} \text { for } 16 \mathrm{~d} \\ & \\ & 2 \mathrm{~g} \mathrm{l}^{-1} \text { for } 12 \mathrm{~d} \\ & 2 \mathrm{~g} \mathrm{l}^{-1} \text { for } 16 \mathrm{~d}\end{array}$

Theronts

Trophonts*
Not effective $-19 \%$ mortality

Effective $-51 \cdot 5 \%, 77 \cdot 5 \%, 79 \%$ and $96 \cdot 5 \%$ mortality respectively

Not effective $-100 \%$ medicated fish high number of trophonts

Tojo-Rodriguez and

Santamarina-Fernandez (2001)

Partially effective $-65 \%$ trophont reduction

Shinn et al. (2005)

Effective $-80 \cdot 2 \%$ trophonts reduction

Partially effective $-71.9 \%$ trophonts reduction

Effective $-93 \cdot 3 \%$ trophonts reduction

Effective - no trophonts on treated fish

Effective - no trophonts on treated fish

Partially effective $-75 \%$ medicated fish free of infection

Effective $-100 \%$ mortality in less than $15 \mathrm{sec}$

Not effective - no details

Partially effective $-\sim 50 \%$ mortality

Effective - 95\% mortality

Effective - 98\% mortality

Not effective $-0 \%$ mortality

Effective $-100 \%$ mortality

$100 \%$ theronts killed; trophonts $57 \%$ lower than controls*

$(8 \cdot 7 \pm 15 \cdot 1 \%$ fish survive $v$ s $0 \%$ of control fish

Theronts $74 \cdot 9 \%$ and trophonts $89 \cdot 8 \%$ lower than

controls*

$96 \cdot 7 \pm 4 \cdot 7 \%$ fish survive $v s \quad 66 \cdot 7 \pm 47 \cdot 1 \%$ of control fish)

$100 \%$ theronts and protomonts mortality*

$(96 \cdot 7 \pm 4 \cdot 7 \%$ fish survive $v s 0 \%$ of control fish)

$100 \%$ theronts and protomonts mortality*

$96 \cdot 7 \pm 4 \cdot 7 \%$ fish survive $v s \quad 66 \cdot 7 \pm 47 \cdot 1 \%$ of control

fish)

Tojo-Rodriguez and

Santamarina-Fernandez (2001)

Farley and Heckmann (1980)

Rahkonen and Koski (2002)

Shinn et al. (2005)

Lahnsteiner and

Weismann (2007)

Mifsud and Rowland (2008) 


\begin{tabular}{|c|c|c|c|c|}
\hline Compound & Dose & $\begin{array}{l}\text { Host/parasite } \\
\text { stage }\end{array}$ & Efficacy & Reference \\
\hline & $3 \mathrm{~g} \mathrm{l}^{-1}$ for $16 \mathrm{~d}$ & & $\begin{array}{l}100 \% \text { theronts and protomonts mortality* } \\
(100 \% \text { fish survive vs } 66 \cdot 7 \pm 47 \cdot 1 \% \text { of control fish) }\end{array}$ & \\
\hline & $3 \mathrm{~g}^{-1}$ daily for $20 \mathrm{~d}$ (static tanks) & I. punctatus & Not effective - all treated fish died & $\begin{array}{l}\text { Tieman and Goodwin } \\
(2001)\end{array}$ \\
\hline & $4 \mathrm{~g} \mathrm{l}^{-1}$ for $45 \mathrm{~d}$ & Rhamdia quelen & $\begin{array}{l}24 \cdot 7 \% \text { of the treated fish survived versus } 8 \cdot 1 \% \text { of the } \\
\text { control fish }\end{array}$ & Miron et al. (2003) \\
\hline & $4 \mathrm{~g}^{-1}$ daily for $30 \mathrm{~d}$ & R. quelen & $\begin{array}{l}\text { 'Effective' - reduction in the number of trophonts on } \\
\text { treated fish }\end{array}$ & Garcia et al. (2007) \\
\hline & $5 \mathrm{~g} \mathrm{l}^{-1}$ for $14 \mathrm{~d}$ at $11-18^{\circ} \mathrm{C}$ & B. bidyanus & Effective - no trophonts visible on treated fish & $\begin{array}{l}\text { Selosse and Rowland } \\
(1990)\end{array}$ \\
\hline & $5 \mathrm{~g} \mathrm{l}^{-1}$ for $8 \mathrm{~d}$ at $24^{\circ} \mathrm{C}$ & B. bidyanus & Effective - no trophonts visible on treated fish & \\
\hline & $5 \mathrm{~g} \mathrm{l}^{-1}$ for $7 \mathrm{~d}$ at $19-22^{\circ} \mathrm{C}$ & Maccullochella peeli & $\begin{array}{l}\text { Effective }- \text { no trophonts visible on treated fish } \\
\text { Effective }- \text { no trophonts visible on treated fish }\end{array}$ & \\
\hline & 10 and $15 \mathrm{~g} \mathrm{l}^{-1}$ for $20 \mathrm{~min}$ & $\begin{array}{l}\text { O. mykiss } \\
\text { S. fontinalis } \\
\text { S. trutta }\end{array}$ & Not effective - no details & Balta et al. (2008) \\
\hline & $20 \mathrm{~g}^{-1}$ for $1 \mathrm{~h}$ for $5 \mathrm{~d}$ & O. mykiss & $\begin{array}{l}\text { Partially effective }-60 \% \text { of treated fish survived, no } \\
\text { infections on } \mathrm{d} 1 \text { but a high infection on } \mathrm{d} 3\end{array}$ & $\begin{array}{l}\text { Lahnsteiner and } \\
\text { Weismann (2007) }\end{array}$ \\
\hline & $20 \mathrm{~g} \mathrm{l}^{-1}$ for $1 \mathrm{~h}$ for $5 \mathrm{~d}$ & C. carpio & $\begin{array}{l}\text { Not effective }-0 \% \text { survival on treated fish } \uparrow \text {; no } \\
\text { infections } \mathrm{d} 1 \\
\text { but high infection on d } 3\end{array}$ & \\
\hline & $20 \mathrm{~g} \mathrm{l}^{-1}$ for $20 \mathrm{~min}$ & $\begin{array}{l}\text { O. mykiss } \\
\text { S. fontinalis } \\
\text { S. trutta }\end{array}$ & $\begin{array}{l}\text { 'Effective' - reduction in the number of trophotns on } \\
\text { treated fish }\end{array}$ & Balta et al. (2008) \\
\hline \multirow[t]{5}{*}{ In-feed } & $0 \cdot 3-1 \cdot 0 \%$ feed for $3-11 \mathrm{~d}$ & C. carpio & 'Effective' - no details & $\begin{array}{l}\text { Rahkonen and Koski } \\
(2002)\end{array}$ \\
\hline & $1 \cdot 2 \%$ feed for $30 \mathrm{~d}$ & R. quelen & $\begin{array}{l}\text { Not effective-high mortality within the treated } \\
\text { groups }\end{array}$ & Garcia et al. (2007) \\
\hline & $2 \cdot 5 \%$ feed for $30 \mathrm{~d}$ & & $\begin{array}{l}\text { Not effective-high mortality within the treated } \\
\text { groups }\end{array}$ & \\
\hline & $5.0 \%$ feed for $30 \mathrm{~d}$ & & Not effective-high mortality on treated groups & \\
\hline & $6 \cdot 0 \%$ feed for $30 \mathrm{~d}$ & & Not effective - high mortality on treated groups & \\
\hline \multicolumn{5}{|c|}{ Sodium percarbonate } \\
\hline \multirow[t]{5}{*}{ In vitro } & $12 \cdot 5 \mathrm{mg}^{-1}$ for $3 \mathrm{~h}$ & Theronts/tcysts & Effective on theronts but not effective on tomocysts & Jensen et al. (2001) \\
\hline & $12 \cdot 5 \mathrm{mg} \mathrm{l}^{-1}$ for $24 \mathrm{~h}$ & Tomocysts & Not effective $-11 \%$ mortality & \\
\hline & $\begin{array}{l}13 \mathrm{mg} \mathrm{l}^{-1} \text { for few hours (no details) } \\
63 \mathrm{mg} \mathrm{l}^{-1} \text { for } 1 \mathrm{~h}\end{array}$ & & Effective on theronts but not effective on tomocysts & Buchmann et al. (2003) \\
\hline & $512 \mathrm{mg} \mathrm{l}^{-1}$ for $<1 \mathrm{~h}$ & Protomonts & Effective $-100 \%$ mortality & Heinecke and Buchmann \\
\hline & $256 \mathrm{mg} \mathrm{l}^{-1}$ for $<1 \mathrm{~h} 30 \mathrm{~min}$ & & Effective $-100 \%$ mortality & \\
\hline
\end{tabular}


$128 \mathrm{mg} \mathrm{l}^{-1}$ for $<4 \mathrm{~h} 15 \mathrm{~min}$

$0 \cdot 5 \mathrm{mg} \mathrm{l}^{-1}$ for $1 \cdot 5,3$ and $15 \mathrm{~h}$

$2 \cdot 5 \mathrm{mg} \mathrm{l}^{-1}$ for $1 \cdot 5,3$ and $15 \mathrm{~h}$

$8 \mathrm{mg} \mathrm{l}^{-1}$ for $30-60 \mathrm{~min}$

$8 \mathrm{mg} \mathrm{l}^{-1}$ for $1 \cdot 5 \mathrm{~h}$

$8 \mathrm{mg} \mathrm{l}^{-1}$ for $2 \mathrm{~h}$

$8 \mathrm{mg} \mathrm{l}^{-1}$ for $2 \cdot 5 \mathrm{~h}$

$8 \mathrm{mg} \mathrm{l}^{-1}$ for $3 \mathrm{~h}$

$8 \mathrm{mg} \mathrm{l}^{-1}$ for $\sim 5 \mathrm{~h}\left(11-12^{\circ} \mathrm{C}\right)$

$8 \mathrm{mg} \mathrm{l}^{-1}$ for $\sim 2 \mathrm{~h} 20 \mathrm{~min}\left(21-22^{\circ} \mathrm{C}\right)$

$12.5 \mathrm{mg} \mathrm{l}^{-1}$ for $1.5 \mathrm{~h}$

$12.5 \mathrm{mg} \mathrm{l}^{-1}$ for 3 and $15 \mathrm{~h}$

$16 \mathrm{mg} \mathrm{l}^{-1}$ for $\sim 1 \mathrm{~h} 20 \mathrm{~min}\left(11-12^{\circ} \mathrm{C}\right)$

$16 \mathrm{mg} \mathrm{l}^{-1}$ for $\sim 1 \mathrm{~h} 40 \mathrm{~min}\left(21-22^{\circ} \mathrm{C}\right)$

$32 \mathrm{mg} \mathrm{l}^{-1}$ for $\sim 1 \mathrm{~h} 10 \min \left(11-12^{\circ} \mathrm{C}\right)$

$32 \mathrm{mg} \mathrm{l}^{-1}$ for $\sim 30 \mathrm{~min}\left(21-22^{\circ} \mathrm{C}\right)$

$62.5 \mathrm{mg} \mathrm{l}^{-1}$ for $1.5,3$ and $15 \mathrm{~h}$

$64 \mathrm{mg} \mathrm{l}^{-1}$ for $\sim 30 \mathrm{~min}\left(11-12{ }^{\circ} \mathrm{C}\right)$

$64 \mathrm{mg} \mathrm{l}^{-1}$ for $\sim 10 \mathrm{~min}\left(21-22^{\circ} \mathrm{C}\right)$

$312.5 \mathrm{mg} \mathrm{l}^{-1}$ for $1 \cdot 5,3$ and $15 \mathrm{~h}$

$1562 \cdot 5 \mathrm{mg} \mathrm{l}^{-1}$ for $1 \cdot 5,3$ and $15 \mathrm{~h}$

In vivo-bath

Concentration not specified for $20 \mathrm{~min}$ every 2 nd and 3 rd day

\section{Sulfachlorpyrazine}

In vitro

$100 \mathrm{mg} \mathrm{l}^{-1}$ for $3 \mathrm{~h}$

$100 \mathrm{mg} \mathrm{l}^{-1}$ for $24 \mathrm{~h}$

$100 \mathrm{mg} \mathrm{l}^{-1}$ for $24 \mathrm{~h}$

Sulfaquinoxaline

In vitro

$200 \mathrm{mg} \mathrm{l}^{-1}$ for $2 \mathrm{~h}$

$200 \mathrm{mg} \mathrm{l}^{-1}$ for $3 \mathrm{~h}$, day 6 p.i.

$1000 \mathrm{mg} \mathrm{kg}^{-1}$ for $8 \mathrm{~d}$

In-feed

Thiophanate

In vivo - In-feed

$40 \mathrm{~g} \mathrm{~kg}^{-1}$ feed for $10 \mathrm{~d}$
Effective $-100 \%$ mortality

Not effective $-<50 \%$ mortality

Not effective $-<50 \%$ mortality

Not effective $-\sim 0 \%$ mortality

Not effective - 40\% mortality

Partially effective $-\sim 70 \%$ mortality

Partially effective $-\sim 70 \%$ mortality

Effective - 100\% mortality

Effective $-100 \%$ mortality

Effective $-100 \%$ mortality

Not effective $-<50 \%$ mortality

Partially effective $->50 \%$ mortality

Effective $-100 \%$ mortality

Effective $-100 \%$ mortality

Effective $-100 \%$ mortality

Effective $-100 \%$ mortality

Partially effective $->50 \%$ mortality

Effective $-100 \%$ mortality

Effective $-100 \%$ mortality

Partially effective $->50 \%$ mortality

Partially effective $->50 \%$ mortality

O. mykiss

Not effective - no details

Not effective - high survival rate after $3 \mathrm{~h}$

Not effective - high survival rate after $24 \mathrm{~h}$

Adults**

Tomocysts

Protomonts

Not effective-high survival rate after $24 \mathrm{~h}$

Not effective $-12 \cdot 5 \%$ mortality after $2 \mathrm{~h}$; protomonts surviving treatment

developed normally

Not effective - all protomonts developed normally

Not effective - all protomonts developed normally

$4 \%$ fish mortality on day 6 p.i.; no feed

unpalatability

Not effective $-100 \%$ medicated fish high number of trophonts Santamarina-Fernandez 2001)

Buchman et al. (2003)

Bruzio and Buchmann (2010)

Heinecke and Buchmann (2009)

Buchman et al. (2003)

Heinecke and Buchmann (2009)

Buchman et al. (2003)

Heinecke and Buchmann 2009)

Buchman et al. (2003)

Rahkonen and Koski (2002)

Wahli et al. (1993) (1994)

Tojo-Rodriguez et al (1994)
O. mykiss
Tojo-Rodriguez and 


\begin{tabular}{|c|c|c|}
\hline Compound & Dose & $\begin{array}{l}\text { Host/parasit } \\
\text { stage }\end{array}$ \\
\hline \multirow[t]{3}{*}{ In vitro } & $\begin{array}{l}10 \mu \mathrm{g} \mathrm{ml}^{-1} \text { for } 2 \mathrm{~h} \\
200 \mathrm{mgl}^{-1} \text { for } 2 \mathrm{~h}\end{array}$ & Protomonts \\
\hline & $<50 \mathrm{mg} \mathrm{l}^{-1}$ for $10 \mathrm{~h}$ & Trophonts* \\
\hline & $10 \mu \mathrm{g} \mathrm{ml}^{-1}$ for $2 \mathrm{~h}$ & Theronts \\
\hline \multirow[t]{3}{*}{ In vivo-bath } & $\begin{array}{l}\mathrm{d} 110 \mathrm{mg} \mathrm{l}^{-1}(2 \mathrm{~h}) \\
\mathrm{d} 2 \text { and d} 320 \mathrm{mg} \mathrm{l}^{-1}(1 \mathrm{~h}) \\
\mathrm{d} 110 \mathrm{mg} / 1(4 \mathrm{~h}) \\
\mathrm{d} 3 \text { and d5 } 10 \mathrm{mg} / 1(4 \mathrm{~h}) \\
1 \mu \mathrm{g} \mathrm{ml}^{-1} \text { for } 4 \cdot 5 \mathrm{~h} \\
5 \mu \mathrm{g} \mathrm{ml}^{-1} \text { for } 4 \cdot 5 \mathrm{~h}\end{array}$ & $\begin{array}{l}\text { Various spp } \\
\text { Various spp } \\
\text { A. rostrata } \\
\text { A. rostrata }\end{array}$ \\
\hline & $10 \mu \mathrm{g} \mathrm{ml}^{-1}$ for $2 \mathrm{~h}$ & \\
\hline & $\begin{array}{l}10 \mu \mathrm{g} \mathrm{ml}^{-1}, 2 \mathrm{~h}(1 \mathrm{st} \mathrm{d}) \\
20 \mu \mathrm{g} \mathrm{ml}^{-1}, 1 \mathrm{~h}(2 \mathrm{nd} \mathrm{d}) \\
20 \mu \mathrm{g} \mathrm{m}^{-1}, 2 \mathrm{~h}(3 \mathrm{rd} \mathrm{d}) \\
5 \mathrm{mg} \mathrm{l}^{-1} \\
10 \mathrm{mg} \mathrm{l}^{-1} \\
20 \mathrm{mg} \mathrm{l}^{-1} \\
50 \mathrm{mg} \mathrm{l}^{-1} \\
200 \mathrm{mg} \mathrm{l}^{-1} \text { for } 3 \mathrm{~h} \text {, day } 6 \text { p.i. }\end{array}$ & $\begin{array}{l}\text { A. rostrata } \\
\text { O. mykiss }\end{array}$ \\
\hline In-feed & $1000 \mathrm{mg} \mathrm{kg}^{-1}$ for $8 \mathrm{~d}$ & O. mykiss \\
\hline
\end{tabular}

Tramisol (6S)-6-phenyl-2,3,5,6-tetrahydroimidazo[2,1-b][1,3]thiazole) In vitro $100 \mathrm{mg} \mathrm{l}^{-1}$ for $2 \mathrm{~min}$

Tricaine methanesulfonate (TM)

In vitro $\quad 50 \mathrm{mg} \mathrm{l}^{-1}$ buffered with $\mathrm{NaCaCO}$

(time of exposure not specified)

$50 \mathrm{mg} \mathrm{l}^{-1}$ not buffered (time of exposure

not specified)

$150 \mathrm{mg} \mathrm{l}^{-1}$ buffered with $\mathrm{Na} \mathrm{CaCO}_{3}$

for $2-3$ min

$150 \mathrm{mg} \mathrm{l}^{-1}$ buffered with $\mathrm{NaCaCO}$

(time of exposure not specified)

$150 \mathrm{mg}^{-1}$ not buffered for $2-3 \mathrm{~min}$
Trophozoites*

Efficacy

Reference

Effective $-100 \%$ mortality

Effective $-0 \%$ mortality but protomonts did not develop

Not effective - trophonts developed normally

Not effective $-0 \%$ mortality

Trophonts affected but not theronts

$100 \%$ trophonts killed but theronts not affected

Not effective-no details

Not effective - one third of the parasites dropped off the fish within $24 \mathrm{~h}$. New infections established within $2 \mathrm{~d}$

Effective - two thirds of the parasites dropped off the fish within $24 \mathrm{~h}$, fish were free from new infections over the following 14 days

'Effective' - no details

Schmahl et al. (1989)

Not effective - toxic, after 5 h $100 \%$ fish mortality Not effective - toxic, after $3 \cdot 5 \mathrm{~h} 100 \%$ fish mortality Not effective-toxic, after 2 h $100 \%$ fish mortality Not effective - toxic, after 2 h $100 \%$ fish mortality

Not effective - all trophonts developed normally

Not effective - all trophonts developed normally

Schmahl et al. (1989)

Tojo-Rodriguez et al. (1994)

Lahnsteiner and

Weismann (2007)

Schmahl et al. (1989)

Mehlhorn et al. (1988)

Mehlhorn et al. (1988)

Schmahl et al. (1989)

From et al. (1992)

Tojo-Rodriguez et al. (1994)

Tojo-Rodriguez et al. (1994)

Effective $-50 \%$ mortality after $2 \mathrm{~h} ; 100 \%$ mortality post exposure

Not effective $-4 \cdot 9 \%$ mortality

Post and Vesley (1983)

Protomonts

Not effective $-4.9 \%$ mortality

$\mathrm{Xu}$ et al. (2008)

Not effective $-1 \cdot 1 \%$ mortality

Not effective $-1 \cdot 8 \%$ mortality

Not effective $-9 \cdot 2 \%$ mortality

Not effective $-6 \cdot 1 \%$ mortality 
$150 \mathrm{mg} \mathrm{l}^{-1}$ not buffered (time of exposure

not specified)

$300 \mathrm{mg} \mathrm{l}^{-1}$ buffered with $\mathrm{Na} \mathrm{CaCO}_{3}$

(time of exposure not specified)

$300 \mathrm{mg}^{-1}$ not buffered (time of exposure

not specified)

Triclabendazole (5-choloro-6-(2, 3-dichlorophenoxy)-2-methylthio-1H- benzimidazole)

In vivo-In-feed $20 \mathrm{~g} \mathrm{~kg}^{-1}$ feed for $10 \mathrm{~d} \quad$ O. mykiss

Triclabendazole $+\beta$-cyclodextrin (ratio $1: 2$ )

In vivo-In-feed $10 \mathrm{~g} \mathrm{~kg}^{-1}$ feed for $10 \mathrm{~d}$

$20 \mathrm{~g} \mathrm{~kg}^{-1}$ feed for $10 \mathrm{~d}$

O. mykiss

Vitamin C

See entry for ascorbate-2-phosphate

Vitamin E

See entry for d-1-alpha-tocopheryl acetate

Violet C

In vivo-bath $\quad 0.01 \mathrm{mg} \mathrm{l}^{-1}$ for $2 \mathrm{~d}$
$0.02 \mathrm{mg} \mathrm{l}^{-1}$ for $6 \mathrm{~d}$

$0.02 \mathrm{mg} \mathrm{l}^{-1}$ for $6 \mathrm{~d}$
$50 \mathrm{mg} \mathrm{l}^{-1}$ for $30 \mathrm{~min}$

C. carpio

Not effective $-9 \cdot 9 \%$ mortality

Not effective $-7 \cdot 3 \%$ mortality

Effective $-100 \%$ mortality

Not effective $-100 \%$ medicated fish with $>50$ trophonts

Partially effective $-58 \%$ reduction in trophont number compared to control

Partially effective $-42 \%$ reduction in trophont

Luzardo-Álavarez et al (2003)

number compared to control

\section{Virkon S}

See the entry for potassium persulfate + sodium dodecylbenzosulfonate + malic acid + sulfamic acid based formulation

Wofasteril $\mathbb{R}$

See entry peracetic Acid (PAA) + hydrogen peroxide + acetic acid $\left(40 \% \mathrm{PAA}+15 \% \mathrm{H}_{2} \mathrm{O}_{2}+25 \% \mathrm{AA}\right)$

Abbreviations: d: days; h: hours; inf.: infection; p.i.: post-infection; *authors use the term 'throphont/trophozites' for the free-swimming stage which exited the fish host; ** authors used the term 'adults' for the free-swimming stage which exited the fish host; $\uparrow$ : toxic to fish; " carp/trout/eels/ornamental fish. 
Table 2. Management strategies tested against infections of Ichthyophthirius multifiliis Fouquet, 1876

(A strategy is regarded as being partially effective if it kills $50-80 \%$, and effective if it kills $\geqslant 80 \%$ of the stages under test. Mortality refers to the parasite stages unless otherwise stated.) Electrotherapy

In vitro-Trophozoides*

Electrode type

Carbon

Carbon

Carbon

Carbon

Carbon

Carbon

Carbon

Copper

Copper

Steel hardware cloth 150-240

Steel hardware cloth 150

Mechanical filtration

In vitro-protomonts

Mesh size $(\mu \mathrm{m})$

500

300

160

Mechanical removal of the tomocysts

In vitro-protomonts

Lining surface

Crystal polysterin

Polypropylene-based plastic

Polyethylene-based plastic

Chlovar chlorinated rubber

In vivo-commercial raceways in O. mykiss hatchery

(Suction head + lining of the bottom of the raceways)

$$
\begin{aligned}
& \text { Visit number } \\
& 1 \text { (after } 2 \text { weeks) } \\
& 2 \text { (after } 4 \text { weeks) } \\
& 3 \text { (after } 6 \text { weeks) } \\
& 4 \text { (after } 8 \text { weeks) } \\
& 5 \text { (after } 10 \text { weeks) }
\end{aligned}
$$

$\begin{array}{lr}\text { Current } & \text { D } \\ & \\ 150-350 & 5 \\ & \\ 200-350 & 5 \\ & 3 \\ & 3 \\ & 3 \\ & 3 \\ & 3 \\ 135-200 & 5 \\ 135 & 5 \\ 160-400 & 5 \\ 340 & 5 \\ \end{array}$

Duration (s)

Efficacy

Not effective $-14 \cdot 35 \%$ mortality after $24 \mathrm{~h}$

Not effective $-7 \cdot 09 \%$ mortality after $24 \mathrm{~h}$

Effective $-100 \%$ mortality

Effective $-100 \%$ mortality

Effective $-100 \%$ mortality

Effective $-100 \%$ mortality

Effective $-100 \%$ mortality

Effective $-100 \%$ mortality after $24 \mathrm{~h}$

Effective $-100 \%$ mortality after $24 \mathrm{~h}$

Not effective $-2.99 \%$ mortality after $24 \mathrm{~h}$

Not effective $-0 \cdot 87 \%$ mortality after $24 \mathrm{~h}$

Efficacy

Not effective $-0 \%$ protomonts filtered out

Not effective $-6 \%$ protomonts filtered out

Not effective $-22 \%$ protomonts filtered out

Effective $-100 \%$ protomonts filtered out
Farley and

Heckmann (1980)

Heinecke and

Buchmann (2009)

Efficacy

Not effective $-9 \cdot 8 \%$ mortality

Effective - $90 \cdot 2 \%$ mortality

Partially effective $-76 \cdot 5 \%$ mortality

Not effective $-46 \cdot 6 \%$ mortality

Efficacy

No infection in control and experimental raceways

No infection in control and experimental raceways

Low infection levels in both control and experimental raceways

Effective $-92 \%$ reduction in trophont numbers compared to the control

Effective $-99 \%$ reduction in trophont numbers compared to the control
Shinn et al. (2009)

Shinn et al. (2009) 
(Suction head stopped, only lining of the bottom of the raceways) 6 (after 12 weeks)

UV light

In vivo- fish species not specified

Number of UV bulbs used (UV light generated)

$1\left(91900 \mu \mathrm{W} \mathrm{s} \mathrm{cm}^{-2}\right)$

$2\left(183800 \mu \mathrm{W} \mathrm{s} \mathrm{cm}^{-2}\right)$

Water flow

In vivo-experimental raceways of I. punctatus fingerlings

$\begin{array}{lc}\begin{array}{l}\text { Fish density } \\ \left(\text { no. } \mathrm{L}^{-1}\right)\end{array} & \left.\begin{array}{l}\text { Flow rate } \\ (\mathrm{L} \mathrm{min}\end{array}{ }^{-1}\right) \\ 0 \cdot 33 & 5 \\ 0 \cdot 25 & 15 \\ 0 \cdot 25 & 25 \\ 0 \cdot 33-0 \cdot 66 & 5 \\ 0 \cdot 33-0 \cdot 66 & 25 \\ 0 \cdot 33-0 \cdot 66 & 45\end{array}$

$0 \cdot 33-0 \cdot 66$

In vivo-production raceways of $I$. punctatus fingerlings
$0 \cdot 89-1 \cdot 29$
2800

$>2800$
Partially effective $-54 \%$ reduction in trophont numbers compared to the control

Efficacy

Not effective $-82 \cdot 81 \%$ fish mortality

Effective $-1 \cdot 33 \%$ fish mortality

Effective $-0 \cdot 7 \%$ fish mortality

Velocity

Turn-over Efficacy

$\left(\mathrm{cm} \min ^{-1}\right)$

$\left(\right.$ no. $\left.\mathrm{h}^{-1}\right)$

$4 \cdot 1$

$0 \cdot 5$
Not effective $-100 \%$ mortality of infected fish

Not effective $-52 \%$ mortality of infected fish

Effective $-14 \%$ mortality of infected fish

Not effective $-100 \%$ mortality of infected

fish

Effective $-9 \%$ mortality of treated fish

Effective $-7 \%$ mortality of treated fish

Gratzek et al. (1983)

Bodensteiner et al.

(2000)

Effective - no trophonts observed

Abbreviations: s: seconds, *authors use the term 'trophozites' for the free-swimming stage which exited the fish host. 


\section{REFERENCES}

Aihua, L. and Buchmann, K. (2001). Temperature and salinitydependent development of a Nordic strain of Ichthyophthirius multifiliis from rainbow trout. Fournal of Applied Ichthyology 17, 173-276.

Alderman, D. J. (1985). Malachite green: a review. Fournal of Fish Diseases 8, 289-298.

Balta, F., Kayis, S. and Altinok, I. (2008). External protozoan parasites in three trout species in the Eastern Black Sea region of the Turkey: intensity, seasonality and their treatments. Bulletin of European Association of Fish Pathologists 28, 157-162.

Bisharyan, Y., Chen, Q., Hossain, M. M., Papoyan, A. and Clark, T. G. (2003). Cadmium effects on Ichthyophthirius: evidence for metal-sequestration in fish tissues following administration of recombinant vaccines. Parasitology 123 (Suppl.) S87-S93.

Bodensteiner, L. R., Sheenan, R. J., Willis, P. S., Brandenburg, A. M. and Lewis, W. M. (2000). Flowing water: an effective treatment for ichthyophthiriasis. Fournal of Aquatic Animal Health 12, 209-219.

Boyd, C. E. (1990). Water Quality In Ponds For Aquaculture. Alabama Agricultural Experiment Station, Auburn University, Auburn, AL, USA. Boyd, C. E. (2005). Copper treatment controls phytoplankton. Global Aquaculture Alliance 8, 69-70.

Brown, E. M. and Gratzek, J. B. (1980). Fish Farming Handbook. Food Bait, Tropicals and Goldfish. AVI Publishing Company, Westport, USA. Bruzio, M. and Buchmann, K. (2010). The effect of peracetic acid products on parasites causing white spot diseases. Fish Farmer 33, 25-27. Bryce, D. I. M., Croshaw, B., Hall, J. E., Holland, V. R. and Lessel, B. (1978). The activity and safety of the antimicrobial agent bronopol (2-bromo2-nitropropan-1,3-diol). Fournal of the Society of Cosmetic Chemists 29, 3-24. Buchmann, K. and Bresciani, J. (1997). Parasitic infections in pondreared rainbow trout Oncorhynchus mykiss in Denmark. Diseases of Aquatic Organisms 28, 125-138.

Buchmann, K., Jensen, P. B. and Kruse, K. D. (2003). Effects of sodium percarbonate and garlic extract on Ichthyophthirius multifiliis theronts and tomocysts: In vitro experiments. North American Yournal of Aquaculture 65 21-24

Buchmann, K., Bresciani, J. and Jappe, C. (2004). Effects of formalin treatment on epithelial structure and mucous cell densities in rainbow trout, Oncorhynchus mykiss (Walbaum), skin. Fournal of Fish Diseases 27, 99-104. Burkart, M. A., Clark, T. G. and Dickerson, H. W. (1990). Immunization of channel catfish, Ictalurus punctatus Rafinesque, against Ichthyophthiriu multifiliis (Fouquet): killed versus live vaccines. Fournal of Fish Diseases 13 401-410.

Cardeilhac, P. and Whitaker, B. (1988). Copper treatments: uses and precautions. Veterinary Clinics of North America 18, 435-448

Colorni, A., Ullal, A., Heinisch, G. and Noga, E. J. (2008). Activity of the antimicrobial polypeptide piscidin 2 against fish ectoparasites. Fournal of Fish Diseases 31, 423-432.

Cross, D. G. (1972). A review of the methods to control ichthyophthiriasis. Progressive Fish Culturist 34, 165-170.

Culp, S. J. (2004). NTP technical report on the toxicity studies on malachite green chloride and leucomalachite green administered in feed to $\mathrm{F} 344 / \mathrm{N}$ rats and B6C3F1 mice. US Department of Health and Human Services June 2004. National Toxicology Program, Toxicity report series. NIH Publication No. 04-4416.

Culp, S. J. and Beland, F.A. (1996). Malachite green: a toxicological review. Fournal of American College Toxicology 15, 219-238.

Deilhac, P. and Whitaker, B. (1988). Copper treatments: uses and precautions. Veterinary Clinics of North America 18, 435-448.

Dickerson, H.W. (2006). Ichthyophthirius multifiliis and Cryptocaryon irritans (Phylum Ciliophora) In Fish Diseases and Disorders, Vol. 1 Protozoan and Metazoan infections (ed. Woo, P. T. K.), pp. 116-153. CAB International, Cambridge, MA, USA.

Dolezelova, P., Macova, S., Plhalova, L., Pistekova, V., Svobodova, Z., Bedanova, I. and Voslarova, E. (2009). Comparison of the sensitivity of different fish species to medical substances. Neuroendocrinology Letters 30, 248-52.

Duffort, G., Houeix, N., Manier, N. and Troise, A. (2010). Institut National de l'EnviRonnement Industriel et des risSques (INERIS)- Fiche de donnees toxicologiques et environnementales des substances chimiques. Formaldéhyde. INERIS.

Ekamen, A. P., Obiekezie, A., Kloas, W. and Knopf, K. (2004). Effects of crude extracts of Mucuna pruriens (Fabaceae) and Carica papaya (Caricaceae) against the protozoan fish parasite Ichthyophthirius multifiliis Parasitology Research 92, 361-366.

Elsayed, E. E., El Dien, N. E. and Mahmoud, M. A. (2006) Ichthyophthiriasis: various fish susceptibility or presence of more than one strain of the parasite? Nature and Science 4, 5-13.
Ewing, M. S., Kocan, K. M. and Ewing, S. A. (1983). Ichthyophthirius multifiliis: morphology of the cyst wall. Transactions of the American Microscopical Society 102, 122-128.

Fallang, A., Ramsay, J. M., Sevatdal, S., Burka, J. F., Jewess, P., Hammell, K. L. and Horsbergs, T. E. (2004). Evidence for occurrence of an organophosphate-resistant type of acetylcholinesterase in strains of sea lice (Lepeophtheirus salmonis Krøyer). Pest Management Science 60, 1163-1170.

Farley, D. G. and Heckmann, R. (1980). Attempts to control Ichthyophthirius multifiliis Fouquet (Ciliophora: Orphryoglnidae) by chemotherapy and electrotherapy. Fournal of Fish Diseases 3, 203-212.

From, J., Karas, N. and Vordermeier, T. (1992). Trials with toltrazuril against Ichthyophthirius multifiliis Fouquet, 1876. Bulletin of European Association of Fish Pathologists 12, 137-138.

Gaikowski, M.P., Densmore, C. L. and Blazer, V.S. (2009) Histopathology of repeated, intermittent exposure of chloramine-T to walleye (Sander vitreum) and (Ictalurus punctalus) channel catfish. Aquaculture 287, 28-34.

Garcia, L., Becker, A. G., Copatti, C. E. and Baldisserotto, B. (2007) Salt in food and water as a supportive therapy of Ichthyophthirius multifiliis infestation on silver catfish, Rhamdia quelen, fingerlings. Fournal of World Aquaculture Society 38, 1-11.

Goodwin, A. E. and Straus, D. L. (2006). Solid and liquid formulations of copper sulphate: efficacy at high and low alkalinities. North American Fournal of Aquaculture 68, 359-363.

Gratzek, J. B., Gilbert, J. P., Lohr, A. L., Shotts, E. B. and Brown, J. (1983). Ultraviolet light control of Ichthyophthirius multifiliis Fouquet in a closed fish culture recirculation system. Fournal of Fish Diseases 6, 145-153. Guest, C. W. (1983). Control of Ichthyophthirius in peacock bass fingerlings. Progressive Fish Culturist 45, 57.

Heinecke, R. D. and Buchmann, K. (2009). Control of Ichthyophthirius multifiliis using a combination of water filtration and sodium percarbonate: dose-response studies. Aquaculture 288, 32-35.

Henderson, A. L., Schmitt, T. C., Heinze, T. M. and Cerniglia, C. E. (1997). Reduction of malachite green to leucomalachite green by intestinal bacteria. Applied Environmental Microbiology 63, 4099-4101.

Herber, T. (2009). Organic compounds used in aquaculture. In Hormones and Pharmaceuticals Generated by Concentrated Animal Feeding Operations, Emerging Topics in Ecotoxicology 1 (ed. Shore, L. S. and Pruden, A.), pp. 95-120. Springer Science and Business Media, New York, USA.

Herwig, N. (1979). Handbook of Drugs and Chemicals used in the Treatment of Fish Diseases. Charles C. Thomas Publisher, Springfield, USA.

Heuer, O. E., Kruse, H., Grave, K., Collignon, P., Karunasagar, I. and

Angulo, F. J. (2009). Human health consequences of use of antimicrobial agents in aquaculture. Clinical Infectious Diseases 49, 1248-1253.

Hines, R. S. and Spira, D. T. (1974). Ichthyophthiriasis in the mirror carp Cyprinus carpio L. V. Acquired immunity. Fournal of Fish Biology 6 373-378.

Hoffman, G. L. and Meyer, F. P. (1974). Parasites of Freshwater Fishes. A Review of their Control Treatment. TFH Publication, Inc Neptune City, USA.

Hohreiter, D. W. and Rigg, D. K. (2001). Derivation of ambient water quality criteria for formaldehyde. Chemosphere 45, 471-486.

Jensen, P. B., Kruse, K. D., Kristensson, R. T. and Buchmann, K. (2001). The effect of sodium percabonate (BioCare SPC) on Ichthyophthirius multifiliis and Gyrodactylus in rainbow trout. Biomar Newsletter 3, 2.

Jørgensen, T. R., Larsen, T. B. and Buchmann, K. (2009). Parasite infections in recirculated rainbow trout (Oncorhynchus mykiss) farms. Aquaculture 289, 91-94.

Johnson, M. D. and Sharma, K. D. (1999). Kinetic and mechanism of the reduction of ferrate by one-electron reductants. Inorganica Chimica Acto 293, 229-233

Kayis, S., Ozcelep, T., Capkin, E. and Altinok, I. (2009). Protozoan and metazoan parasites of cultured fish in Turkey and their applied treatments. The Israeli Fournal of Aquaculture 61, 93-102.

Kurovskaya, L. Y. (2005). Effect of violet C antiseptic on the numbers of ectoparasites and some physiological and biochemical indices of carps. Hydrobiological Fournal 41, 95-102.

Lauridsen, J. H. and Buchmann, K. (2010). Effects of short and long-term glucan feeding of rainbow trout (Salmonidae) on the susceptibility to Ichthyophthirius multifiliis infections. Acta Ichthyologica et Piscatoria 40, 61-66. Lahnsteiner, F. and Weismann, T. (2007). Treatment of ichthyophthiriasis in rainbow trout and common carp with common and alternative therapeutics. Fournal of Aquatic Animal Health 19, 186-194.

Lees, F., Baillie, M., Gettinby, G. and Revie, C. W. (2008). The efficacy of emamectin benzoate against infestations of Lepeophtheirus salmonis on farmed Atlantic salmon (Salmo salar L.) in Scotland, 2002-2006. PLoS ONE 3, e1549. 
Ling, F., Luo, Q., Wang, J.L., Wang, Y.P., Wang, W. B. and Gong, X. N. (2009). Effects of the 'all-fish' GH (growth hormone) transgene expression on resistance to Ichthyophthirius multifiliis infections in common carp, Cyprinus carpio L. Aquaculture 292, 1-5.

Ling, F., Wang, J. G., Liu, O.F., Li, M., Ye, L. T. and Gong, X. N. (2010). Prevention of Ichthyophthirius multifiliis infestation in goldfish (Carassius auratus) by postassium ferrate (VI) treatment. Veterinary Parasitology 168, 212-216.

Ling, K. H., Sin, Y. M. and Lam, T. J. (1993). Effect of copper sulfate on ichthyophthiriasis (white spot disease) in goldfish (Carassius auratus). Aquaculture 118, 23-25.

Lom, J. and Dyková, I. (1992). Protozoan Parasites of Fishes. Developments in Aquaculture and Fisheries Science, Vol. 26. Elsevier, Amsterdam.

Luzardo-Álvarez, A., Martínez-Mazagatos, J., SantamarinaFernández, M. T., Otero-Espinar, F. J. and Blanco-Méndez, J. (2003). Oral pharmacological treatments for Ichthyophthirius of rainbow trout (Oncorhynchus mykiss). Aquaculture 220, 15-25.

Ma, J. and Liu, W. (2002). Effectiveness and mechanism of potassium ferrate (VI) preoxidation for algae removal by coagulation. Water Research 36, 871-878.

Matthews, R. A. (1994). Ichthyophthirius multifiliis Fouquet, 1876: infection and proactive response within the fish host. In Parasitic Diseases of Fish (ed. Pike, A. W. and Lewis, J. W.), Samara Publishing Ltd., Dyfed. Matthews, R.A. (2005). Ichthyophthirius multifiliis Fouquet and ichthyophthiriosis in freshwater teleosts. Advances in Parasitology 59, 159-241

Mehlhorn, H., Schmahl, G. and Heberkorn, A. (1988). Toltrazuril effective against a broad spectrum of protozoan parasites. Parasitology Research 75, 64-66.

Meinelt, T., Pietrock, M., Burnison, K. and Steinberg, C. (2005). Formaldehyde toxicity is altered by calcium and organic matter. Fournal of Applied Ichthyology 21, 121-124.

Meinelt, T., Matzke, S., Stüber, A., Pietrock, M., Wienke, A., Mitchell, A. J. and Straus, D. L. (2009). Toxicity of peracetic acid (PAA) to tomonts of Ichthyophthirius multifiliis. Diseases of Aquatic Organisms 86, 51-56.

Mifsud, C. and Rowland, S. J. (2008). Use of salt to control ichthyophthiriosis and prevent saprolegniosis in silver perch, Bidyanus bidyanus. Aquaculture Research 39, 1175-1180.

Miron, D. S., Silva, L. V. F., Golombieski, J. I. and Baldisserotto, B. (2003). Efficacy of different salt $(\mathrm{NaCl})$ concentrations in the treatment of Ichthyophthirius multifiliis-infected silver catfish, Rhamdia quelen, fingerlings. Fournal of Applied Aquaculture 14, 155-161.

Moore, J. M. (2005). Comparison of copper toxicity to channel catfish, Ictalurus punctatus, and blue catfish, I. furcatus, fingerlings. Fournal of Applied Aquaculture 17, 77-84.

Noble, A. C. and Summerfelt, S. T. (1996). Diseases encountered in rainbow trout cultured in recirculating systems. Annual Review of Fish Diseases 6, 65-92.

Noga, E. J. (2010). Fish Disease: Diagnosis and Treatment. Wiley-Blackwell. Novartis (2002). Pyceze: Technical dossier. Information for Farmers. Novartis Animal Vaccines. UK.

Pedersen, L.F., Pedersen, P. B., Nielsen, J. L. and Nielsen, P. H. (2009). Peracetic acid degradation and effects on nitrification in recirculating aquaculture systems. Aquaculture 296, 246-254.

Picón-Camacho, S. M., Taylor, N. G.H., Bron, J. E., Guo, F. C. and Shinn, A.P. (2011a). Effects of continuous exposure to low doses of bronopol on the infection dynamics of Ichthyophthirius multifiliis (Ciliophora), parasitising rainbow trout (Oncorhynchus mykiss Walbaum). Veterinary Parasitology (in the Press).

Picón-Camacho, S. M., Marcos-Lopez, M., Beljean, A., Debeaume, S. and Shinn, A.P. (2011b). In vitro assessment of the chemotherapeutic action of a specific hydrogen peroxide, peracetic and peroctanoic acid based formulation against the free-living stages of Ichthyophthirius multifiliis (Ciliophora). Parasitology Research.

Pieters, N., Brunt, J., Austin, B. and Lyndon, A. R. (2008). Efficacy of infeed probiotics against Aeromonas bestiarum and Ichthyophthirius multifiliis skin infections in rainbow trout (Oncorhynchus mykiss, Walbaum). Fournal of Applied Microbiology 105, 723-732.

Pillay, T. V.R. and Kutty, M. N. (2005). Aquaculture: Principles and Practices. Blackwell Publishing, Oxford.

Post, G. and Vesely, K. R. (1983). Administration of drugs by hyperosmotic or vacuum infiltration or surfactant immersion ineffective for control of intradermally encysted Ichthyophthirius multifiliis. Progressive Fish Culturist 45, 164-166.

Pottinger, T. G. and Day, J. G. (1999). A Saprolegnia parasitica challenge system for rainbow trout: assessment of Pyceze as an anti-fungal control agent for both fish and ova. Diseases of Aquatic Organisms 36, 129-141.
Powell, M. and Harris, J. (2004). Influence of oxygen on the toxicity of chloramine-T to Atlantic salmon smolts in freshwater and seawater. Fournal of Aquatic Animal Health 16, 83-92.

Rábago-Castro, J. L., Sanchez, J. G., Pérez-Castaneda, R. and González-González, A. (2006). Effects of the prophylactic use of Romet ${ }^{\circledR}-30$ and copper sulfate on growth, condition and feeding indices in channel catfish (Ictalurus punctatus). Aquaculture 253, 343-349.

Rahkonen, R. and Koski, P. (2002). Post malachite green: alternative strategies for fungal infections and white spot disease. Bulletin of European Association of Fish Pathologists 22, 152-157.

Rapp, J. (1995). Treatments of rainbow trout (Oncorhynchus mykiss Walb.) fry infected with Ichthyophthirius multifiliis by oral administration of dimetrazole. Bulletin of European Association of Fish Pathologists 15, 67-69. Rintamäki-Kinnunen, P. and Valtonen, E. T. (1997). Epizootiology of protozoans in farmed salmonids in northern latitudes. International Fournal of Parasitology 27, 89-99.

Rintamäki-Kinnunen, P., Rahkonen, M., MannermaaKeränen, A. L., Suomalainen, L. R., Mykrä, H. and Valtonen, E. T. (2005a). Treatment of ichthyophthiriasis after malachite green. I. Concrete tanks at salmonid farms. Diseases of Aquatic Organisms 64, 69-76.

Rintamäki-Kinnunen, P., Rahkonen, M., Mykrä, $H$. and Valtonen, E. T. (2005b). Treatment of ichthyophthiriasis after malachite green. II. Earth ponds at salmonids farms. Diseases of Aquatic Organisms 66, $15-20$

Rowland, S. J., Misfud, C., Nixon, M., Read, P. and Landos, M. (2009). Use of formalin and copper to control ichthyophthiriosis in the Australian freshwater fish silver perch (Bidyanus bidyanus Mitchell). Aquaculture Research 40, 44-54.

Ruider, S., Schmal, G., Mehlhorn, H., Scmidt, H. and Ritter, G. (1997). Effects of different malachite green derivates and metabolites on the fish ectoparasite, Ichthyophthirius multifiliis, Fouquet 1876 (Hymenostomatida, Ciliophora). European Fournal of Protistology 33, 375-388.

Sanabria, C., Diamant, A. and Zilberg, D. (2009). Effects of commonly used disinfectants and temperature on swim bladder non-inflation in freshwater angelfish, Pterophyllum scalare (Lichtenstein). Aquaculture 292, $158-165$.

Schmidt, L. J., Gaikowski, M.P. and Gingerich, W. H. (2006). Environmental Assessment for the Use of Hydrogen Peroxide in Aquaculture for Treating External Fungal and Bacterial Diseases of Cultured Fish and Fish Eggs. USGS. Science for a Changing World. pp. 180 .

Schlenk, D., Gollon, J. L. and Griffin, B. R. (1998). Efficacy of copper sulfate for the treatment of ichthyophthiriasis in channel catfish. Fournal of Aquatic Animal Health 10, 390-396.

Schlotfeld, H. J. (1993). Chemikalien zur therapie bei nutzfischen nun zugelassen! Standard-Zulassungen. Fisch Teichwirt 12, 421-424.

Schlotfeld, H. J. (1998). Therapienotstand bei fischen in der Bundesrepublik Deutschland. Fisch Teichwirt 2, 42-47.

Schmahl, G., Mehlhorn, H. and Taraschewski, H. (1989). Treatment of fish parasites. 5. The effects of sym. triazinone (toltrazuril) on fish parasitic Ciliophora (Ichthyophthirius multifiliis Fouquet, 1876, Apiosoma amoebea Grenfell, 1884, Trichodina sp. Ehrenberg, 1831). European Fournal of Protistology 24, 152-161.

Schmahl, G., Schmidt, H. and Ritter, G. (1996). The control of ichthyopthiriasis by a medicated food containing quinine: efficacy test and ultrastructure investigations. Parasitology Research 82, 697-705.

Schnick, R.A. (1988). The impetus to register new therapeutants for aquaculture. Progressive Fish Culturist 50, 190-196.

Selosse, P. M. and Rowland, S. J. (1990). Use of common salt to treat ichthyophthiriasis in Australian warm water fishes. Progressive Fish Culturist 52, 124-127.

Shinn, A., Wootten, R., Sommerville, C. and Conway, D. (2001). Putting the squeeze on whitespot. Trout News 32, 20-25.

Shinn, A.P., Wootten, R. and Sommerville, C. (2003a). Alternative compounds for the treatment of Ichthyophthirius multifiliis infecting rainbow trout. Trout News 35, 38-41.

Shinn, A. P., Wootten, R., Côte, I. and Sommerville, C. (2003b) Efficacy of selected oral chemotherapeutants against Ichthyophthirius multifiliis (Ciliophora: Ophyroglenidae) infecting rainbow trout Oncorhynchus mykiss. Diseases of Aquatic Organisms 55, 17-22.

Shinn, A., Taylor, N. and Wootten, R. (2005). Development of a management system for the control of Ichthyophthirius multifiliis. Trout News 40, 21-25.

Shinn, A. P., Picón-Camacho, S. M., Bawden, R. and Taylor, N. G. H. (2009). Mechanical control of Ichthyophthirius multifiliis Fouquet, 1876 (Ciliophora) in a rainbow trout hatchery. Aquaculture Engineering 41, 152-157. 
Shinn, A.P., Picón-Camacho, S. M., Bron, J. E., Conway, D., Yoon, G. H., Guo, F. C. and Taylor, N. G. H. (2011). The anti-protozoa activity of bronopol on the key life-stages of Ichthyophthirius multifiliis Fouquet, 1876 (Ciliophora). Veterinary Parasitology (in press).

Sommerset, I., Krossøy, B., Biering, E. and Frost, P. (2005). Vaccines for fish in aquaculture. Expert Reviews of Vaccines 4, 89-101.

Straus, D. L. and Griffin, B. R. (2001). Prevention of an initial infestation of Ichthyophthirius multifiliis in channel catfish and blue tilapia by potassium permanganate treatment. North American Fournal of Aquaculture 63, 11-16. Srivastava, S., Sinha, R. and Roy, D. (2004). Toxicological effects of malachite green. Aquatic Toxicology 66, 319-329.

Straus, D. L. and Griffin, B. R. (2002). Efficacy of potassium permanganate in treating ichthyophthiriasis in channel catfish. Fournal of Aquatic Animal Health 14, 145-148.

Straus, D. L. (1993). Prevention of Ichthyophthirius multifiliis infestation in channel catfish fingerlings by copper sulfate treatment. Fournal of Aquatic Animal Health 5, 152-154.

Straus, D. L. (2008). Comparison of copper sulphate concentrations to control ichthyophthiriasis in fingerling channel catfish. Fournal of Applied Aquaculture 20, 272-284.

Straus, D. L. and Meinelt, T. (2009). Acute toxicity of peracetic acid (PAA) formulations to Ichthyophthirius multifiliis theronts. Parasitology Research 104, 1237-1241

Straus, D. L., Hossain, M. M. and Clark, T. G. (2009). Strain differences in Ichthyophthirius multifiliis to copper toxicity. Diseases of Aquatic Organisms 83, 31-36.

Sudová, E., Straus, D. L., Wienke, A. and Meinelt, T. (2010). Evaluation of continuous 4-day exposure to peracetic acid as a treatment for Ichthyophthirius multifiliis. Parasitology Research 106, 539-542.

Swennes, A. G., Findly, R. C. and Dickerson, H. W. (2007). Crossimmunity and antibody responses to different immobilisation serotypes of Ichthyophthirius multifiliis. Fish Shellfish Immunology 22, 589-597.

Tieman, D. M. and Goodwin, A. E. (2001). Treatments of ich infestations in channel catfish evaluated under static and flow-through water conditions. North American Fournal of Aquaculture 63, 293-299.

Tojo, J. L., Santamarina, M. T., Ubeira, F. M., Leiro, J. and Sanmartin, M. L. (1994). Trials for the control of ichthyophthiriosis in rainbow trout (Oncorhynchus mykiss). Bulletin of European Association of Fish Pathologists 14, 148-152.

Tojo-Rodriguez, J. L. and Santamarina-Fernandez, M. T. (2001) Attempts at oral pharmacological treatment of Ichthyophthirius multifilii in rainbow trout, Oncorhynchus mykiss (Walbaum). Fournal of Fish Diseases 24, 249-252.

Toksen, E. and Nemli, E. (2010). Oral treatment trials on telescope fish (Carassius auratus) experimentally infected with Ichthyophthirius multifiliis (Fouquet, 1876). Bulletin of European Association of Fish Pathologists 30 $48-52$.
Treves-Brown, K. M. (2000). Applied Fish Pharmacology. Kluwer Academic Publishers, Dordrecht.

Tucker, C. S. (1987). Acute toxicity of potassium permanganate to channel catfish fingerlings. Aquaculture 60, 93-98.

Ullal, A. J., Litaker, R. W. and Noga, E. J. (2008). Antimicrobial peptides derived from haemoglobin are expressed in epithelium of channel catfish (Ictalurus punctatus, Rafinesque). Developmental and Comparative Immunology 32, 1301-1312.

Ullal, A. J. and Noga, E. J. (2010). Antiparasitic activity of the antimicrobial pepetide $\mathrm{Hb} \beta \mathrm{P}-1$, a member of the $\beta$-haemoglobin peptide family. Fournal of Fish Diseases 33, 657-664.

Valtonen, E. T. and Koskivaara, M. (1994). Relationships between the parasites of some wild and cultured fishes in two lakes and a fish farm in central Finland. International Yournal of Parasitology 24, 109-118.

Wagner, G. (1960). Der Entwicklungszyklus von Ichthyophthirius multifiliis Fouquet und der Einfluss physikalischer und chemischer aussenfaktoren. Zeitschrift Fisherei 9, 425-443.

Wahli, T., Streiff, K. and Meier, W. (1985). Influence of ascorbic acid on Ichthyophthirius multifiliis infections in trout. Bulletin of European Association of Fish Pathologists 4, 86-87.

Wahli, T., Schmitt, M. and Meier, W. (1993). Evaluation of alternatives to malachite green oxalate as a therapeutant for ichthyopthirius in ranbow trout, Oncorhynchus mykiss. Fournal of Applied Ichthyology 9, 237-249.

Wahli, T., Frischknecht, R., Schmitt, M., Gabaudan, J., Verlhac, V. and Meier, W. (1995). A comparison of the effect of silicone coated ascorbic acid and ascorbyl phosphate on the course of ichthyophthiriosis in rainbow trout, Oncorhynchus mykiss (Walbaum). Fournal of Fish Diseases 18, 347-355.

Wahli, T., Verlhac, V., Gabaudan, J., Schüep, W. and Meier, W. (1998). Influence of combined vitamins $\mathrm{C}$ and $\mathrm{E}$ on non-specific immunity and diseases resistance of rainbow trout, Oncorhynchus mykiss (Walbaum) Fournal of Fish Diseases 21, 127-137.

Wooster, G. A., Martinez, C. M. and Bowser, P. R. (2005). Human health risks associated with formalin treatments used in aquaculture: Initial study. North American Fournal of Aquaculture 67, 111-113.

World Health Organization, International Agency for Research on Cancer (2006). Formaldehyde, 2- butoxyethanol and 1-tert-butoxypropan2 -ol. IARC monographs on the evaluation of carcinogenic risks to humans Volume 88. World Health Organization, International Agency for Research on Cancer, Lyon.

Xu, D. H., Schoemaker, C. A. and Klesius, P.H. (2008). Effect of tricaine methanesulfonate on survival and reproduction of the fish ectoparasite Ichthyophthirius multifiliis. Parasitology Research 103, 979-982. Yao, J.Y., Shen, J.Y., Li, X. L., Xu, Y., Hao, G. J., Pan, X.Y. Wang, G. X. and Yin, W. L. (2010). Effect of sanguinarine from the leaves of Macleaya cordata against Ichthyophthirius multifiliis in grass carp (Ctenopharyngodon idella). Parasitology Research 107, 1035-1042. 